\title{
The Calculus of Boundary Variations and the Dielectric Boundary Force in the Poisson-Boltzmann Theory for Molecular Solvation
}

\author{
Bo Li * Zhengfang Zhang ${ }^{\dagger} \quad$ Shenggao Zhou ${ }^{\ddagger}$
}

October 9, 2020

\begin{abstract}
In a continuum model of the solvation of charged molecules in an aqueous solvent, the classical Poisson-Boltzmann (PB) theory is generalized to include the solute point charges and the dielectric boundary that separates the high-dielectric solvent from the low-dielectric solutes. With such a setting, we construct an effective electrostatic freeenergy functional of ionic concentrations, where the solute point charges are regularized by a reaction field. We prove that such a functional admits a unique minimizer in a class of admissible ionic concentrations and that the corresponding electrostatic potential is the unique solution to the boundary-value problem of the dielectric-boundary PB equation. The negative first variation of this minimum free energy with respect to variations of the dielectric boundary defines the normal component of the dielectric boundary force. Together with the solute-solvent interfacial tension and van der Waals interaction forces, such boundary force drives an underlying charged molecular system to a stable equilibrium, as described by a variational implicit-solvent model. We develop an $L^{2}$-theory for the continuity and differentiability of solutions to elliptic interface problems with respect to boundary variations, and derive an explicit formula of the dielectric boundary force. With a continuum description, our result of the dielectric boundary force confirms a molecular-level prediction that the electrostatic force points from the high-dielectric and polarizable aqueous solvent to the charged molecules. Our method of analysis is general as it does not rely on any variational principles.
\end{abstract}

Keywords: Molecular solvation, Poisson-Boltzmann equation, electrostatic free energy, point charges, dielectric boundary force, the calculus of boundary variations.

*Department of Mathematics, University of California, San Diego, 9500 Gilman Drive, MC 0112, La Jolla, CA 92093-0112, USA. Email: bli@math.ucsd.edu

${ }^{\dagger}$ College of Science, Hangzhou Dianzi University, Hangzhou, Zhejiang 310018, China. Email: zhengfangzhang@hdu.edu.cn

${ }^{\ddagger}$ Department of Mathematics and Mathematical Center for Interdiscipline Research, Soochow University, 1 Shizi Street, Suzhou 215006, Jiangsu, China. Email: sgzhou@suda.edu.cn 


\section{Contents}

1 Introduction $\quad 2$

2 The Poisson-Boltzmann Equation and Free-Energy Functional 7

2.1 Assumptions and Auxiliary Functions . . . . . . . . . . . . . . . . . . 7

2.2 The Poisson-Boltzmann Equation . . . . . . . . . . . . . . . . . . 11

2.3 Electrostatic Free-Energy Functional of Ionic Concentrations . . . . . . . . . . 12

3 Dielectric Boundary Force

3.1 Electrostatic Free Energy of a Dielectric Boundary . . . . . . . . . . . . . . . 15

3.2 Definition and Formula of the Dielectric Boundary Force . . . . . . . . . . . 18

4 Some Lemmas: The Calculus of Boundary Variations 20

4.1 Properties of the Transformation $T_{t} \ldots \ldots \ldots \ldots$. . . . . . . . . . . 20

4.2 Tangential Force . . . . . . . . . . . . . . . . . . . . 21

4.3 Continuity and Differentiability ................... 24

5 Proof of Theorem 3.2

$\begin{array}{ll}\text { Acknowledgements } & 48\end{array}$

$\begin{array}{ll}\text { References } & 48\end{array}$

\section{Introduction}

The classic Poisson-Boltzmann (PB) theory provides a continuum description of electrostatic interactions in an ionic solution through the $\mathrm{PB}$ equation $2,6,17,23,27]$

$$
\nabla \cdot \varepsilon \nabla \psi-B^{\prime}(\psi)=-\rho \quad \text { in } \Omega_{0},
$$

where $\Omega_{0} \subseteq \mathbb{R}^{3}$ is the region of the ionic solution, $\varepsilon$ is the dielectric coefficient, $\rho: \Omega_{0} \rightarrow \mathbb{R}$ represents the density of fixed charges, and $\psi: \Omega_{0} \rightarrow \mathbb{R}$ is the electrostatic potential. In (1.1), the function $B: \mathbb{R} \rightarrow \mathbb{R}$ is defined by

$$
B(s)=\beta^{-1} \sum_{j=1}^{M} c_{j}^{\infty}\left(e^{-\beta q_{j} s}-1\right) \quad \forall s \in \mathbb{R},
$$

where $\beta=\left(k_{\mathrm{B}} T\right)^{-1}$ with $k_{\mathrm{B}}$ the Boltzmann constant and $T$ the temperature, $M$ is the total number of ionic species, $c_{j}^{\infty}$ is the bulk ionic concentration of the $j$ th ionic species, and $q_{j}=z_{j} e$ is the charge of an ion of the $j$ th species with $z_{j}$ the valence of such an ion and $e$ the elementary charge. The PB equation (1.1) is a combination of Poisson's equation

$$
\nabla \cdot \varepsilon \nabla \psi=-\left(\rho+\sum_{j=1}^{M} q_{j} c_{j}\right) \quad \text { in } \Omega_{0},
$$


where $c_{j}: \Omega_{0} \rightarrow[0, \infty)$ is the ionic concentration of the $j$ th ionic species, and the Boltzmann distributions for the equilibrium ionic concentrations

$$
c_{j}(x)=c_{j}^{\infty} e^{-\beta q_{j} \psi(x)}, \quad x \in \Omega_{0}, j=1, \ldots, M .
$$

In modeling charged molecules (such as proteins) in an aqueous solvent (i.e., water or salted water) within an implicit-solvent (i.e., continuum-solvent) framework, the PB theory is generalized to include the point charges of the charged molecules and a dielectric boundary that separates the high-dielectric solvent region from the low-dielectric solute region 7,14 , 16, 34, 45, 48. To be more specific, let us assume that the entire solvation system occupies a region $\Omega \subseteq \mathbb{R}^{3}$. It is the union of three disjoint parts: the region of solutes (i.e., charged molecules) $\Omega_{-}$; the region of aqueous solvent $\Omega_{+}$; and the solute-solvent interface or dielectric boundary $\Gamma$, which is a closed surface with possibly multiple components, that separates $\Omega_{-}$ and $\Omega_{+}$; cf. Figure 1 . We denote by $n$ the unit normal to the boundary $\Gamma$ pointing from $\Omega_{-}$ to $\Omega_{+}$and also the exterior unit normal to $\partial \Omega$, the boundary of $\Omega$. The solute region $\Omega_{-}$ contains all the solute atoms that are located at $x_{1}, \ldots, x_{N}$ and that carry partial charges $Q_{1}, \ldots, Q_{N}$, respectively, where $N \geq 1$ is a given integer. The solvent region $\Omega_{+}$is the region of ionic solution, similar to $\Omega_{0}$ in (1.1). As before, we assume that there are $M$ species of ions in the solvent region $\Omega_{+}$with the valence $z_{j}$, charge $q_{j}=z_{j} e$, bulk concentration $c_{j}^{\infty}$, and the local concentration $c_{j}: \Omega_{+} \rightarrow[0, \infty)$ for the $j$ ionic species $(j=1, \ldots, M)$. The dielectric coefficients in the solute region $\Omega_{-}$and solvent region $\Omega_{+}$are denoted by $\varepsilon_{-}$and $\varepsilon_{+}$, respectively. Typically, $\varepsilon_{-}=1$ and $\varepsilon_{+}=76 \sim 80$ in the unit of vacuum permittivity. Note that the density of fixed charges is now given by $\rho=\sum_{i=1}^{N} Q_{i} \delta_{x_{i}}$, where $\delta_{x_{i}}$ is the Dirac delta function at $x_{i}$.

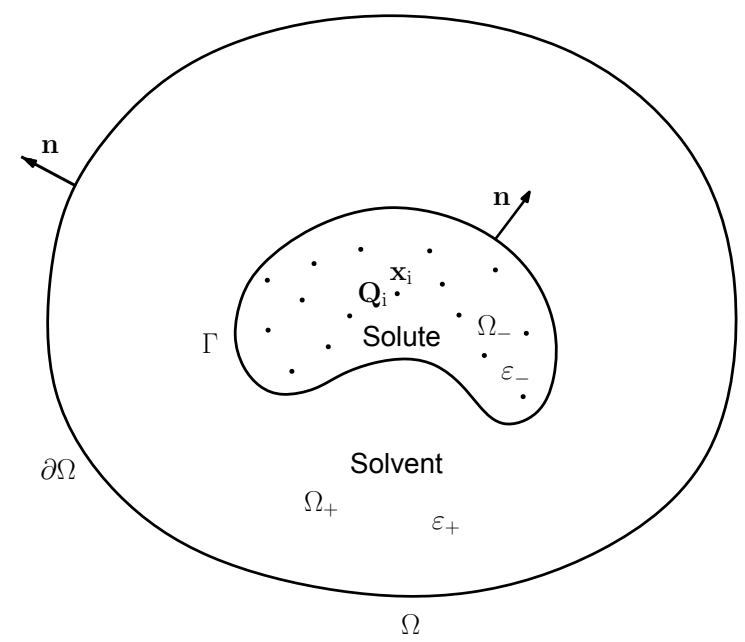

Figure 1: A schematic description of a solvation system with an implicit solvent.

We introduce the dielectric-boundary, electrostatic free-energy functional of the ionic con- 
centrations $c=\left(c_{1}, \ldots, c_{M}\right)$ in the solvent region $\Omega_{+}, 7,24,34,44$

$$
\begin{aligned}
F_{\Gamma}[c]= & \frac{1}{2} \sum_{i=1}^{N} Q_{i}\left(\psi-\hat{\phi}_{\mathrm{C}}\right)\left(x_{i}\right)+\frac{1}{2} \int_{\Omega_{+}}\left(\sum_{j=1}^{M} q_{j} c_{j}\right) \psi d x \\
& +\beta^{-1} \sum_{j=1}^{M} \int_{\Omega_{+}}\left\{c_{j}\left[\log \left(\Lambda^{3} c_{j}\right)-1\right]+c_{j}^{\infty}\right\} d x-\sum_{j=1}^{M} \int_{\Omega_{+}} \mu_{j} c_{j} d x
\end{aligned}
$$

where $\Lambda$ is the thermal de Broglie wavelength, $\mu_{j}$ is the chemical potential for ions of the $j$ th species, and $c_{j}^{\infty}=\Lambda^{-3} e^{\beta \mu_{j}}(j=1, \ldots, M)$. In $(1.3), \psi: \Omega \rightarrow \mathbb{R}$ is the electrostatic potential. It is the unique weak solution to the boundary-value problem of Poisson's equation

$$
\begin{cases}\nabla \cdot \varepsilon_{\Gamma} \nabla \psi=-\left(\sum_{i=1}^{N} Q_{i} \delta_{x_{i}}+\chi_{+} \sum_{j=1}^{M} q_{j} c_{j}\right) & \text { in } \Omega \\ \psi=\phi_{\infty} & \text { on } \partial \Omega\end{cases}
$$

where the dielectric coefficient $\varepsilon_{\Gamma}: \Omega \rightarrow \mathbb{R}$ is defined by

$$
\varepsilon_{\Gamma}(x)= \begin{cases}\varepsilon_{-} & \text {if } x \in \Omega_{-} \\ \varepsilon_{+} & \text {if } x \in \Omega_{+}\end{cases}
$$

$\chi_{+}=\chi_{\Omega_{+}}$is the characteristic function of $\Omega_{+}$, and $\phi_{\infty}$ is a given function on the boundary $\partial \Omega$. The function $\hat{\phi}_{\mathrm{C}}$ in $(1.3)$ is the Coulomb potential arising from the point charges $Q_{i}$ at $x_{i}(i=1, \ldots, N)$ in the medium with the dielectric coefficient $\varepsilon_{-}$, serving as a reference field. It is given by

$$
\hat{\phi}_{\mathrm{C}}(x)=\sum_{i=1}^{N} \frac{Q_{i}}{4 \pi \varepsilon_{-}\left|x-x_{i}\right|} \quad \forall x \in \mathbb{R}^{3} \backslash\left\{x_{1}, \ldots, x_{N}\right\} .
$$

We prove that the functional $F_{\Gamma}[c]$ has a unique minimizer $c_{\Gamma}=\left(c_{\Gamma, 1}, \ldots, c_{\Gamma, M}\right)$ in a class of admissible concentrations, and derive the equilibrium conditions $\delta_{c_{j}} F_{\Gamma}\left[c_{\Gamma}\right]=0(j=1, \ldots, M)$, which lead to the (modified) Botlzmann distributions

$$
c_{\Gamma, j}=c_{j}^{\infty} e^{-\beta q_{j}\left(\psi_{\Gamma}-\phi_{\Gamma, \infty} / 2\right)} \quad \text { in } \Omega_{+}, j=1, \ldots, M,
$$

where $\psi_{\Gamma}$ is the corresponding electrostatic potential and $\phi_{\Gamma, \infty}: \Omega \rightarrow \mathbb{R}$ is the unique weak solution to the boundary-value problem

$$
\begin{cases}\nabla \cdot \varepsilon_{\Gamma} \nabla \phi_{\Gamma, \infty}=0 & \text { in } \Omega \\ \phi_{\Gamma, \infty}=\phi_{\infty} & \text { on } \partial \Omega\end{cases}
$$

We also prove that $\psi_{\Gamma}$ is the unique solution to the boundary-value problem of the dielectricboundary $\mathrm{PB}$ equation

$$
\begin{cases}\nabla \cdot \varepsilon_{\Gamma} \nabla \psi-\chi_{+} B^{\prime}\left(\psi-\frac{\phi_{\Gamma, \infty}}{2}\right)=-\sum_{i=1}^{N} Q_{i} \delta_{x_{i}} & \text { in } \Omega \\ \psi=\phi_{\infty} & \text { on } \partial \Omega\end{cases}
$$


where $B$ is given in 1.2 ; cf. Theorem 2.1 and Theorem 2.2. With the Boltzmann distributions, the minimum free energy $\min F_{\Gamma}[\cdot]=F_{\Gamma}\left[c_{\Gamma}\right]$ can be expressed via the electrostatic potential $\psi_{\Gamma}$. We shall denote this minimum free energy by $E[\Gamma]$, as ultimately it depends on the dielectric boundary $\Gamma$.

We define the (normal component of the) dielectric boundary force to be $-\delta_{\Gamma} E[\Gamma]$, the negative first variation of the functional $E[\Gamma]$ with respect to the variation of the boundary $\Gamma$. The boundary variation is defined via a smooth vector field. Specifically, let $V: \mathbb{R}^{3} \rightarrow \mathbb{R}^{3}$ be a smooth map vanishing outside a small neighborhood of the dielectric boundary $\Gamma$. Let $x=x(t, X)$ be the solution map of the dynamical system defined by $3,18,30,46$

$$
\frac{d x(t, X)}{d t}=V(x(t, X)) \quad \forall t \in \mathbb{R} \quad \text { and } \quad x(0, X)=X \quad \forall X \in \mathbb{R}^{3} .
$$

Such solution maps define a family of transformations $T_{t}: \mathbb{R}^{3} \rightarrow \mathbb{R}^{3}(t \in \mathbb{R})$ by $T_{t}(X)=$ $x(t, X)$ for any $X \in \mathbb{R}^{3}$. The variational derivative (i.e., the shape derivative) of the functional $E[\Gamma]$ in the direction of $V: \mathbb{R}^{3} \rightarrow \mathbb{R}^{3}$ is defined to be

$$
\delta_{\Gamma, V} E[\Gamma]=\left.\frac{d}{d t} E\left[\Gamma_{t}(V)\right]\right|_{t=0},
$$

if it exists, where $\Gamma_{t}(V)=\{x(t, X): X \in \Gamma\}$ and $E\left[\Gamma_{t}(V)\right]$ is defined similarly using $\Gamma_{t}(V)$ instead of $\Gamma$.

We prove that $\delta_{\Gamma, V} E[\Gamma]$ exists, and is an integral over $\Gamma$ of the product of $V \cdot n$ and some function that is independent of $V$, where $n$ is the unit normal along $\Gamma$, pointing from $\Omega_{-}$to $\Omega_{+}$. This function on $\Gamma$ is identified as the variational derivative of $E[\Gamma]$ and is denoted by $\delta_{\Gamma} E[\Gamma]$. We obtain an explicit formula for $\delta_{\Gamma} E[\Gamma]$. If the boundary value $\phi_{\infty}=0$ on $\Gamma$, then

$$
\delta_{\Gamma} E[\Gamma]=-\frac{1}{2}\left(\frac{1}{\varepsilon_{+}}-\frac{1}{\varepsilon_{-}}\right)\left|\varepsilon_{\Gamma} \partial_{n} \psi_{\Gamma}\right|^{2}+\frac{1}{2}\left(\varepsilon_{+}-\varepsilon_{-}\right)\left|\nabla_{\Gamma} \psi_{\Gamma}\right|^{2}+B\left(\psi_{\Gamma}\right),
$$

where $\psi_{\Gamma}$ is the unique solution to $(1.8), \varepsilon_{\Gamma} \partial_{n} \psi_{\Gamma}$ is the common value from both sides of $\Gamma$, and $\nabla_{\Gamma}=(I-n \otimes n) \nabla$ (with $I$ the $3 \times 3$ identity matrix) is the tangential derivative along $\Gamma$. Additional terms arise from a general, inhomogeneous boundary value $\phi_{\infty}$; cf. Theorem 3.2 .

To describe the electrostatic free energy with point charges and to prove the main theorem, Theorem 3.2, we introduce various auxiliary functions that are weak solutions to the boundary-value problems of the operator $-\Delta$ or $-\nabla \cdot \varepsilon_{\Gamma} \nabla$, with or without the point charges $\sum_{i=1}^{N} Q_{i} \delta_{x_{i}}$ and with homogeneous or inhomogeneous Dirichlet boundary conditions. We also prove several lemmas, Lemmas 4.14 .5 , on the calculus of boundary variations. Lemma 4.1 is of its own interest. It states that if the vector field $V$ satisfies $V \cdot n=0$ on the boundary $\Gamma$, where $n$ is the unit normal along $\Gamma$, then for $|t| \ll 1$ the set $\Gamma_{t}=\Gamma_{t}(V)$ is within an $O\left(t^{2}\right)$-neighborhood of the boundary $\Gamma$. Lemmas 4.24 .5 are on the continuity and differentiability of those functions with respect to boundary variations. Lemma 4.3 states that the " $\Gamma$-derivative" of the function $\phi_{\Gamma, \infty}$ which is defined in 1.7$)$ is the unique weak solution 
$\zeta_{\Gamma, V} \in H_{0}^{1}(\Omega)$ to the elliptic interface problem $-\nabla \cdot \varepsilon_{\Gamma} \nabla \zeta_{\Gamma, V}=f$ in $\Omega$, where $f$ involves $\phi_{\Gamma, V}$ and $V$. Moreover,

$$
\frac{\phi_{\Gamma_{t}(V), \infty} \circ T_{t}-\phi_{\Gamma, \infty}}{t} \rightarrow \zeta_{\Gamma, V} \quad \text { in } H^{1}(\Omega) \quad \text { as } t \rightarrow 0 .
$$

Lemma 4.4 and Lemma 4.5 generalize the result to other $\Gamma$-dependent functions, including the electrostatic potential $\psi_{\Gamma}$ that is the unique soltuion to the boundary-value problem of the dielectric-boundary PB equation (1.8).

We now make several remarks on our results. In our model, we use an inhomogeneous Dirichlet boundary condition for the electrostatic potential (cf. (1.4) and (1.8)) that is common in modeling and analysis $[7,34,53]$. The nonzero Dirichlet boundary value leads to an extra term $\phi_{\Gamma, \infty} / 2$ in the Boltzmann distribution and hence in the PB equation (1.8). If there are surface charges on the boundary $\partial \Omega$, then one can also use the Neumann boundary condition for the electrostatic potential on $\partial \Omega$. In that case, the electrostatic energy should include a boundary integral term involving the surface charge density; cf. [36, 41].

If we use the homogeneous Dirichlet boundary condition $\phi_{\infty}=0$ for the electrostatic potential, then the dielectric boundary force points from the high dielectric solvent region $\Omega_{+}$ to the low dielectric solute region $\Omega_{-}$; cf. (1.9). Such prediction of a macroscopic property is consistent with a microscopic picture of molecular forces that charged solute molecules polarize the surrounding aqueous solvent, which is otherwise electrically neutral, generating an additional electric filed that attracts the solvent to the solutes [13]. In the limiting case where the region $\Omega_{+}$is conducting, i.e., the dielectric coefficient in $\Omega_{+}$is infinity, then it is expected that no bounded region $\Omega_{-}$will minimize the sum of the electrostatic energy and the surface energy [42]. If a small, high-dielectric solvent region is surrounded by the low-dielectric solute molecules (such as a few water molecules buried in a protein), then the competition between the solute-solvent interfacial tension force and the dielectric boundary force results an equilibrium solute-solvent interface which is however unstable with long-wave perturbations, as shown in the stability analysis in [8]; cf. also [38]. Such analysis explains partially why water molecules in proteins are metastable [51,52]. It remains open to confirm if the dielectric boundary force still points from the high-dielectric solvent region to the low-dielectric solute region for a general inhomogeneous Dirichlet boundary value $\phi_{\infty}$.

In [4, 5, 50], the authors use the Maxwell stress tensor to define and derive the dielectric boundary force given an electrostatic potential that is determined by the dielectric-boundary $\mathrm{PB}$ equation. The existence of such a stress tensor in the presence of dielectric boundary is implicitly assumed. The shape derivative approach seems first introduced in [35] to define and derive the dielectric boundary force. However, approximations of point charges by smooth functions are made there, and the derivation of the boundary force utilizes heavily on the underlying variational principle that the electrostatic potential extremizes the dielectricboundary PB free-energy functional. This approach is applied to the electrostatic force acting on membranes [43]. Here, we use the direct calculations to derive the boundary force, which is a more general approach.

Our study of the dielectric boundary force is closely related to the development of a variational implicit-solvent model (VISM) for biomolecules [19, 20] (cf. also [9 11, 49, 53, 54]). 
Central in the VISM is an effective free-energy functional of all possible dielectric boundaries that consists mainly of the surface energy of solute molecules, solute-solvent van der Waals interaction energy, and continuum electrostatic free energy. Minimization of the free-energy functional with respect to the dielectric boundary yields optimal solute-solvent interfaces, as well as the solvation free energy. Numerical implementation of such minimization requires a formula of the first variation of the VISM function, particularly, the dielectric boundary force. In [37], the authors use the matched asymptotic analysis to derive the sharp-interface limit of a phase-field VISM [47]. In [15], the authors prove the convergence of the free energy and force in the phase-field VISM to their sharp-interface counterparts. In particular, they prove the general result that the variation of the van der Waals-Cahn-Hilliard functional converges to the mean curvature which is the variation of surface area. The recent work 26] is a detailed analysis of the electrostatics in molecular solvation through different scaling regimes arising from the large-number limit of solute particles.

The rest of the paper is organized as follows: In Section 2, we first state our assumptions and introduce some auxiliary functions. We then prove the existence, uniqueness, and bounds for the solution to the boundary-value problem of the dielectric-boundary PB equation. We finally study the electrostatic free-energy functionals of ionic concentrations and electrostatic potentials, respectively, with a given set of point charges and a dielectric boundary. In Section 3, we reformulate the minimum electrostatic free energy, define the dielectric boundary force, and present the main formula for such force. In Section 4, we prove several lemmas on the calculus of boundary variations. These lemmas are needed for the proof of the main theorem on the dielectric boundary force. Finally, in Section 5 , we prove the main theorem (Theorem 3.2 of the dielectric boundary force.

\section{The Poisson-Boltzmann Equation and Free-Energy Functional}

\subsection{Assumptions and Auxiliary Functions}

Unless otherwise stated, we assume the following throughout the rest of the paper:

A1. The set $\Omega \subset \mathbb{R}^{3}$ is non-empty, bounded, open, and connected. The sets $\Omega_{-} \subset \mathbb{R}^{3}$ and $\Omega_{+} \subset \mathbb{R}^{3}$ are non-empty, bounded, and open, and satisfy that $\overline{\Omega_{-}} \subset \Omega$ and $\Omega_{+}=\Omega \backslash \overline{\Omega_{-}}$. The interface $\Gamma=\partial \Omega_{-}=\overline{\Omega_{-}} \cap \overline{\Omega_{+}}$and the boundary $\partial \Omega$ are of the class $C^{3}$ and $C^{2}$, respectively. The unit normal vector at the boundary $\Gamma$ exterior to $\Omega_{-}$and that at $\partial \Omega$ exterior to $\Omega$ are both denoted by $n$. The $N$ points $x_{1}, \ldots, x_{N}$ for some integer $N \geq 1$ belong to $\Omega_{-}$; cf. Figure 1. Moreover, there exists a constant $s_{0}>0$ such that

$$
\operatorname{dist}(\Gamma, \partial \Omega) \geq s_{0}
$$

A2. All the integer $M \geq 2$, and real numbers $\beta>0, \Lambda>0, Q_{i} \in \mathbb{R}(1 \leq i \leq N), q_{j} \neq 0$ and $\mu_{j} \in \mathbb{R}(1 \leq j \leq M)$, and $\varepsilon_{-}>0$ and $\varepsilon_{+}>0$ are given. Moreover, $\varepsilon_{-} \neq \varepsilon_{+}$. The parameter $c_{j}^{\infty}$ is defined by $c_{j}^{\infty}=\Lambda^{-3} e^{\beta \mu_{j}}(j=1, \ldots, M)$. The parameters $q_{j}$ and $c_{j}^{\infty}$ 
$(1 \leq j \leq M)$ satisfy the condition of charge neutrality

$$
\sum_{j=1}^{M} q_{j} c_{j}^{\infty}=0
$$

A3. The function $B: \mathbb{R} \rightarrow \mathbb{R}$ is defined in $(1.2)$. The function $\varepsilon_{\Gamma} \in L^{\infty}(\Omega)$ is defined in (1.5). The boundary data $\phi_{\infty}$ is the trace of a given function, also denoted by $\phi_{\infty}$, in $C^{2}(\Omega)$. (We use the standard notation for Sobolev spaces and other function spaces; cf. $[1,22,25]$.

Note that the function $B$ defined in $(1.2)$ satisfies that $B \in C^{\infty}(\mathbb{R})$. Since

$$
B^{\prime}(s)=-\sum_{j=1}^{M} q_{j} c_{j}^{\infty} e^{-\beta q_{j} s} \quad \text { and } \quad B^{\prime \prime}(s)=\sum_{j=1}^{M} \beta q_{j}^{2} c_{j}^{\infty} e^{-\beta q_{j} s}>0, \quad \forall s \in \mathbb{R},
$$

the function $B$ is strictly convex, and the charge neutrality 2.2 implies that $B^{\prime}(0)=0$. Hence, $s=0$ is the unique minimum point for $B$ with $B(s)>B(0)=0$ for all $s \neq 0$. By the fact that $M \geq 2$ and the charge neutrality $(2.2)$, there exist some $q_{j}>0$ and some $q_{k}<0$. Hence, $B( \pm \infty)=\infty$. Similar arguments show that $B^{\prime}(\infty)=\infty$ and $B^{\prime}(-\infty)=-\infty$.

We now introduce several auxiliary functions to treat the point-charge singularities, the dielectric discontinuity $\Gamma$, and the inhomogeneous boundary data $\phi_{\infty}$ on $\partial \Omega$. We first recall that the Coulomb field $\hat{\phi}_{\mathrm{C}}$ is defined in 1.6$)$. Let $\hat{\phi} \in \hat{\phi}_{\mathrm{C}}+H^{1}(\Omega)$ be defined by

$$
\int_{\Omega} \varepsilon_{-} \nabla \hat{\phi} \cdot \nabla \eta d x=\sum_{i=1}^{N} Q_{i} \eta\left(x_{i}\right) \quad \forall \eta \in C_{\mathrm{c}}^{1}(\Omega),
$$

where $C_{\mathrm{c}}^{1}(\Omega)$ denotes the class of $C^{1}(\Omega)$-functions that are compactly supported in $\Omega$. Clearly, we can modify the value of $\hat{\phi}$ on a set of zero Lebesgue measure, if necessary, so that $\hat{\phi}$ is a $C^{\infty}$-function in $\Omega \backslash\left\{x_{1}, \ldots, x_{N}\right\}$. Moreover, $\Delta \hat{\phi}=0$ in $\Omega \backslash\left\{x_{1}, \ldots, x_{N}\right\}$ and $\Delta\left(\hat{\phi}-\hat{\phi}_{\mathrm{C}}\right)=0$ in $\Omega$. There are infinitely many such functions. We will only use three of them. One of them is the Coulomb field $\hat{\phi}=\hat{\phi}_{\mathrm{C}}$. The other two are $\hat{\phi}=\hat{\phi}_{0}$ and $\hat{\phi}=\hat{\phi}_{\infty}$. They are uniquely determined by the boundary conditions

$$
\hat{\phi}_{0}=0 \quad \text { on } \partial \Omega \quad \text { and } \quad \hat{\phi}_{\infty}=\phi_{\infty} \text { on } \partial \Omega \text {, }
$$

respectively. Since $\partial \Omega$ is $C^{2}$ and $\phi_{\infty} \in C^{2}(\bar{\Omega})$, we have $\hat{\phi}-\hat{\phi}_{\mathrm{C}} \in H^{2}(\Omega)$; cf. Chapter 8 in 25$]$. Therefore, all these three functions belong to $\hat{\phi}_{\mathrm{C}}+H^{2}(\Omega) \cap C^{\infty}(\Omega) \subset W^{1,1}(\Omega)$.

We remark that $\eta \in C_{\mathrm{c}}^{1}(\Omega)$ in $(2.3)$ can be replaced by $\eta \in H_{0}^{1}(\Omega)$ with $\left.\eta\right|_{\Omega_{-}} \in C^{1}\left(\Omega_{-}\right)$. To see this, we first note that 2.3 holds true if $\hat{\phi}$ is replaced by $\hat{\phi}_{\mathrm{C}}$ (cf. (1.6)). Thus,

$$
\int_{\Omega} \varepsilon_{-} \nabla\left(\hat{\phi}-\hat{\phi}_{\mathrm{C}}\right) \cdot \nabla \eta d x=0 \quad \forall \eta \in H_{0}^{1}(\Omega)
$$


as $\hat{\phi}-\hat{\phi}_{\mathrm{C}} \in H^{1}(\Omega)$ and $C_{\mathrm{c}}^{1}(\Omega)$ is dense in $H^{1}(\Omega)$. If $\eta \in H_{0}^{1}(\Omega)$ also satisfies $\left.\eta\right|_{\Omega_{-}} \in C^{1}\left(\Omega_{-}\right)$, then $\nabla \hat{\phi}_{\mathrm{C}} \cdot \nabla \eta$, hence $\nabla \hat{\phi} \cdot \nabla \eta$, is integrable in $\Omega$. Moreover,

$$
\int_{\Omega} \varepsilon_{-} \nabla \hat{\phi} \cdot \nabla \eta d x=\int_{\Omega} \varepsilon_{-} \nabla \hat{\phi}_{\mathrm{C}} \cdot \nabla \eta d x=\sum_{i=1}^{N} Q_{i} \eta\left(x_{i}\right),
$$

where the second equality follows from straight forward calculations using the definition of $\hat{\phi}_{\mathrm{C}}$ (cf. (1.6)).

We recall that the function $\phi_{\Gamma, \infty} \in H^{1}(\Omega)$ is the unique weak solution to the boundaryvalue problem (1.7), defined by $\phi_{\Gamma, \infty}=\phi_{\infty}$ on $\partial \Omega$ and

$$
\int_{\Omega} \varepsilon_{\Gamma} \nabla \phi_{\Gamma, \infty} \cdot \nabla \eta d x=0 \quad \forall \eta \in H_{0}^{1}(\Omega) .
$$

By the regularity theory, we have, after modifying possibly the value of $\phi_{\Gamma, \infty}$ on a set of zero Lebesgue measure, that

$$
\phi_{\Gamma, \infty} \in C(\bar{\Omega}) \cap W^{1, \infty}(\Omega) \quad \text { and }\left.\quad \phi_{\Gamma, \infty}\right|_{\Omega_{\mathrm{s}}} \in C^{\infty}\left(\Omega_{\mathrm{s}}\right) \cap H^{2}\left(\Omega_{\mathrm{s}}\right) \quad \text { for } s=-,+.
$$

Moreover, there exists a constant $C=C\left(\Omega, \varepsilon_{+}, \varepsilon_{-}, \phi_{\infty}\right)>0$, independent of $\Gamma$, such that

$$
\left\|\phi_{\Gamma, \infty}\right\|_{W^{1, \infty}(\Omega)} \leq C \text {. }
$$

See [25] (Theorem 8.16) and [39] (Theorem 1.1 and the beginning part of proof of Theorem 1.1) (also [12]) for the global $C(\bar{\Omega})$ and $W^{1, \infty}$ regularities, and the $W^{1, \infty}(\Omega)$ estimate, and 32 (Section 16 of Chapter 3) and 28,29] for the piecewise $H^{2}$-regularity. By 2.5), we have

$$
\Delta \phi_{\Gamma, \infty}=0 \quad \text { in } \Omega_{-} \cup \Omega_{+} .
$$

This implies the piecewise $C^{\infty}$-regularity in (2.6). Moreover, since $\phi_{\Gamma, \infty} \in H_{0}^{1}(\Omega)$, routine calculations by 2.5) and the Divergence Theorem imply that [34]

$$
\llbracket \phi_{\Gamma, \infty} \rrbracket_{\Gamma}=0 \quad \text { and } \quad \llbracket \varepsilon_{\Gamma} \partial_{n} \phi_{\Gamma, \infty} \rrbracket_{\Gamma}=0 .
$$

Throughout, for any function $u$ on $\Omega$ that has trace on $\Gamma$, we denote

$$
u^{+}=\left.u\right|_{\Omega_{+}}, \quad u^{-}=\left.u\right|_{\Omega_{-}}, \quad \text { and } \quad \llbracket u \rrbracket_{\Gamma}=u^{+}-u^{-} \quad \text { on } \Gamma .
$$

Let $\hat{\phi}_{\Gamma, \infty} \in \hat{\phi}_{\mathrm{C}}+H^{1}(\Omega)$ be the unique function such that $\hat{\phi}_{\Gamma, \infty}=\phi_{\infty}$ on $\partial \Omega$ and

$$
\int_{\Omega} \varepsilon_{\Gamma} \nabla \hat{\phi}_{\Gamma, \infty} \cdot \nabla \eta d x=\sum_{i=1}^{N} Q_{i} \eta\left(x_{i}\right) \quad \forall \eta \in C_{\mathrm{c}}^{1}(\Omega)
$$

cf. 21, 40]. If $\hat{\phi}=\hat{\phi}_{\mathrm{C}}$, or $\hat{\phi}_{0}$, or $\hat{\phi}_{\infty}$, then 2.11) is equivalent to

$$
\int_{\Omega} \varepsilon_{\Gamma} \nabla\left(\hat{\phi}_{\Gamma, \infty}-\hat{\phi}\right) \cdot \nabla \eta d x=-\left(\varepsilon_{+}-\varepsilon_{-}\right) \int_{\Omega_{+}} \nabla \hat{\phi} \cdot \nabla \eta d x
$$




$$
=\left(\varepsilon_{+}-\varepsilon_{-}\right) \int_{\Gamma} \partial_{n} \hat{\phi} \eta d S \quad \forall \eta \in H_{0}^{1}(\Omega),
$$

where the unit normal $n$ at $\Gamma$ points from $\Omega_{-}$to $\Omega_{+}$. If $\eta \in H_{0}^{1}(\Omega)$ satisfies $\left.\eta\right|_{\Omega_{-}} \in C^{1}\left(\Omega_{-}\right)$, then it follows from 2.12 that

$$
\begin{aligned}
\int_{\Omega} \varepsilon_{\Gamma} \nabla \hat{\phi}_{\Gamma, \infty} \cdot \nabla \eta d x & =\int_{\Omega} \varepsilon_{\Gamma} \nabla \hat{\phi} \cdot \nabla \eta d x-\left(\varepsilon_{+}-\varepsilon_{-}\right) \int_{\Omega_{+}} \nabla \hat{\phi} \cdot \nabla \eta d x \\
& =\int_{\Omega} \varepsilon_{-} \nabla \hat{\phi} \cdot \nabla \eta d x \\
& =\sum_{i=1}^{N} Q_{i} \eta\left(x_{i}\right) .
\end{aligned}
$$

Therefore, we can replace $\eta \in C_{\mathrm{c}}^{1}(\Omega)$ in $(2.11)$ by $\eta \in H_{0}^{1}(\Omega)$ that satisfies $\left.\eta\right|_{\Omega_{-}} \in C^{1}\left(\Omega_{-}\right)$.

By 2.11) and 2.12, we have, after possibly modifying the value of $\hat{\phi}_{\Gamma, \infty}$ on a set of zero Lebesgue measure, that

$$
\begin{array}{lll}
\Delta\left(\hat{\phi}_{\Gamma, \infty}-\hat{\phi}\right)=0 \quad \text { in } \Omega_{-} & \text {and } \quad \Delta \hat{\phi}_{\Gamma, \infty}=0 & \text { in }\left(\Omega_{-} \backslash\left\{x_{1}, \ldots, x_{N}\right\}\right) \cup \Omega_{+}, \\
\llbracket \hat{\phi}_{\Gamma, \infty} \rrbracket_{\Gamma}=0 & \text { and } \quad \llbracket \varepsilon_{\Gamma} \partial_{n} \hat{\phi}_{\Gamma, \infty} \rrbracket_{\Gamma}=0 \quad \text { on } \Gamma .
\end{array}
$$

Moreover, it follows from the elliptic regularity theory [12, 21, 25, 28, 29, 32, 39, 40, that $\hat{\phi}_{\Gamma, \infty}-\hat{\phi} \in C(\bar{\Omega}) \cap W^{1, \infty}(\Omega), \quad\left(\hat{\phi}_{\Gamma, \infty}-\hat{\phi}\right)^{-} \in C^{\infty}\left(\Omega_{-}\right) \cap H^{2}\left(\Omega_{-}\right), \quad \hat{\phi}_{\Gamma, \infty}^{+} \in C^{\infty}\left(\Omega_{+}\right) \cap H^{2}\left(\Omega_{+}\right)$.

Further, then there exists a constant $C>0$ that may depend on $\Omega, x_{i}$ and $Q_{i}(1 \leq i \leq N)$, $\varepsilon_{+}, \varepsilon_{-}, \phi_{\infty}$, and $\hat{\phi}$, but does not depend on $\Gamma$, such that

$$
\left\|\hat{\phi}_{\Gamma, \infty}-\hat{\phi}\right\|_{W^{1, \infty}(\Omega)} \leq C .
$$

These results (2.15) and (2.16) follow from the same arguments used above (cf. the description below (2.7) ) applied to (2.11) with $\eta \in C_{\mathrm{c}}^{1}(\Omega)$ so chosen that the support of $\eta$ is in a neighborhood of $\Gamma$ that excluding the sigularities $x_{i}(i=1, \ldots, N)$.

For any $g \in H^{-1}(\Omega)$, let $L_{\Gamma} g \in H_{0}^{1}(\Omega)$ be the unique weak solution (defined using test functions in $\left.H_{0}^{1}(\Omega)\right)$ to the boundary-value problem

$$
\nabla \cdot \varepsilon_{\Gamma} \nabla L_{\Gamma} g=-g \text { in } \Omega \quad \text { and } \quad L_{\Gamma} g=0 \text { on } \partial \Omega .
$$

This defines a linear, continuous, and self-adjoint operator $L_{\Gamma}: H^{-1}(\Omega) \rightarrow H_{0}^{1}(\Omega)$. The map

$$
g \mapsto\|g\|_{L_{\Gamma}}:=\sqrt{\left\langle g, L_{\Gamma} g\right\rangle_{H^{-1}(\Omega), H_{0}^{1}(\Omega)}}=\left[\int_{\Omega} \varepsilon_{\Gamma}\left|\nabla\left(L_{\Gamma} g\right)\right|^{2} d x\right]^{1 / 2}
$$

defines a norm on $H^{-1}(\Omega)$ which is equivalent to the $H^{-1}(\Omega)$-norm. If $g \in L^{1}(\Omega)$, then we define $g \in L^{1}(\Omega) \cap H^{-1}(\Omega)$ if

$$
\sup \left\{\int_{\Omega} g u d x: u \in H_{0}^{1}(\Omega) \cap L^{\infty}(\Omega) \text { and }\|u\|_{H^{1}(\Omega)}=1\right\}<\infty .
$$


In this case, the action of $g$ on $H_{0}^{1}(\Omega)$ is defined first for any $u \in H_{0}^{1}(\Omega) \cap L^{\infty}(\Omega)$ by the integral of $g u$ over $\Omega$ and then extended for any $u \in H_{0}^{1}(\Omega)$ by 2.19 and the fact that $H_{0}^{1}(\Omega) \cap L^{\infty}(\Omega)$ is dense in $H_{0}^{1}(\Omega)$.

\subsection{The Poisson-Boltzmann Equation}

We now study the well-posedness of the boundary-value problem of the Poisson-Boltzmann (PB) equation (1.8) with a dielectric boundary and point charges.

Definition 2.1. A function $\psi \in \hat{\phi}_{\mathrm{C}}+H^{1}(\Omega)$ is a weak solution to the boundary-value problem of the dielectric-boundary $P B$ equation $(1.8)$, if $\psi=\phi_{\infty}$ on $\partial \Omega, \chi_{+} B^{\prime}\left(\psi-\phi_{\Gamma, \infty} / 2\right) \in L^{1}(\Omega) \cap$ $H^{-1}(\Omega)$, and

$$
\int_{\Omega}\left[\varepsilon_{\Gamma} \nabla \psi \cdot \nabla \eta+\chi_{+} B^{\prime}\left(\psi-\frac{\phi_{\Gamma, \infty}}{2}\right) \eta\right] d x=\sum_{i=1}^{N} Q_{i} \eta\left(x_{i}\right) \quad \forall \eta \in C_{\mathrm{c}}^{1}(\Omega) .
$$

Note that we can replace $\eta \in C_{\mathrm{c}}^{1}(\Omega)$ in $(2.20)$ by $\eta \in H_{0}^{1}(\Omega)$ that satisfies $\eta^{-} \in C^{1}\left(\Omega_{-}\right)$; cf. the remark below (2.12). The theorem below provides the existence and uniqueness of the solution to the boundary-value problem of the dielectric-boundary PB equation, and an equivalent formulation of such a boundary-value problem. These results are essentially proved in [35]. Here we sketch the proof and add some points that are not included in the previous proof due to some minor differences between the current and previous statements. Note that $\hat{\phi}_{\mathrm{C}}+H^{1}(\Omega)=\hat{\phi}_{\Gamma, \infty}+H^{1}(\Omega)$. So, we can replace $\hat{\phi}_{\mathrm{C}}$ by $\hat{\phi}_{\Gamma, \infty}$ in the above definition. Note also that there is a variational principle for the PB equation; cf. Theorem 3.1 .

Theorem 2.1. (1) There exists a unique weak solution $\psi_{\Gamma} \in \hat{\phi}_{\Gamma, \infty}+H_{0}^{1}(\Omega)$ of the boundaryvalue problem of the dielectric-boundary PB equation (1.8). Moreover, after a possible modification of $\psi_{\Gamma}$ on a set of zero Lebesgue measure, $\psi_{\Gamma}-\hat{\phi}_{\Gamma, \infty} \in C(\bar{\Omega}) \cap W^{1, \infty}(\Omega)$, $\left(\psi_{\Gamma}-\hat{\phi}_{\Gamma, \infty}\right)^{-} \in C^{\infty}\left(\Omega_{-}\right) \cap H^{2}\left(\Omega_{-}\right)$, and $\psi_{\Gamma}^{+} \in C^{\infty}\left(\Omega_{+}\right) \cap H^{2}\left(\Omega_{+}\right)$. Further, there exists a constant $C>0$ that may depend on $\Omega, x_{i}$ and $Q_{i}(1 \leq i \leq N), \varepsilon_{+}, \varepsilon_{-}, \phi_{\infty}$, and $B$, but does not depend on $\Gamma$, such that

$$
\left\|\psi_{\Gamma}-\hat{\phi}_{\Gamma, \infty}\right\|_{W^{1, \infty}(\Omega)} \leq C
$$

(2) A function $\psi \in \hat{\phi}_{\Gamma, \infty}+H^{1}(\Omega)$ with $\chi_{+} B^{\prime}\left(\psi-\phi_{\Gamma, \infty} / 2\right) \in L^{1}(\Omega) \cap H^{-1}(\Omega)$ is the weak solution to the boundary-value problem of the dielectric-boundary PB equation (1.8) if and only if it is the unique solution to the following elliptic interface problem:

$$
\begin{cases}\Delta\left(\psi-\hat{\phi}_{\Gamma, \infty}\right)=0 & \text { in } \Omega_{-}, \\ \varepsilon_{+} \Delta \psi-B^{\prime}\left(\psi-\frac{\phi_{\Gamma, \infty}}{2}\right)=0 & \text { in } \Omega_{+}, \\ \llbracket \psi \rrbracket_{\Gamma}=0 \quad \text { and } \quad \llbracket \varepsilon_{\Gamma} \partial_{n} \psi \rrbracket_{\Gamma}=0 & \text { on } \Gamma, \\ \psi=\psi_{\infty} & \text { on } \partial \Omega .\end{cases}
$$


Proof. (1) With $u=\psi-\hat{\phi}_{\Gamma, \infty}$ and by 2.11) and 2.20 , it is equivalent to show that there exists a unique $u_{\Gamma} \in H_{0}^{1}(\Omega)$ such that $\chi_{+} B^{\prime}\left(u_{\Gamma}+\hat{\phi}_{\Gamma, \infty}-\phi_{\Gamma, \infty} / 2\right) \in L^{1}(\Omega) \cap H^{-1}(\Omega)$, and

$$
\int_{\Omega}\left[\varepsilon_{\Gamma} \nabla u_{\Gamma} \cdot \nabla \eta+\chi_{+} B^{\prime}\left(u_{\Gamma}+\hat{\phi}_{\Gamma, \infty}-\frac{\phi_{\Gamma, \infty}}{2}\right) \eta\right] d x=0 \quad \forall \eta \in H_{0}^{1}(\Omega) .
$$

Define

$$
I[u]=\int_{\Omega}\left[\frac{\varepsilon_{\Gamma}}{2}|\nabla u|^{2}+\chi_{+} B\left(u+\hat{\phi}_{\Gamma, \infty}-\frac{\phi_{\Gamma, \infty}}{2}\right)\right] d x \quad \forall u \in H_{0}^{1}(\Omega) .
$$

Since $B \geq 0$ and $B$ is convex, we can use the direct method in the calculus of variations to obtain a unique minimizer $u_{\Gamma} \in H_{0}^{1}(\Omega)$ of the functional $I: H_{0}^{1}(\Omega) \rightarrow[0, \infty]$. Moreover, comparing the values $I\left[u_{\Gamma}\right]$ and $I\left[u_{\Gamma, \lambda}\right]$ for any constant $\lambda>0$ large enough, where $u_{\Gamma, \lambda}=u_{\Gamma}$ if $\left|u_{\Gamma}\right| \leq \lambda$ and $u_{\Gamma, \lambda}=\lambda \operatorname{sign}\left(u_{\Gamma}\right)$ otherwise, we have by the convexity of $B$ that $u_{\Gamma}=u_{\Gamma, \lambda}$ a.e. $\Omega$ for some $\lambda$ independent on $\Gamma$. Hence, $u_{\Gamma} \in L^{\infty}(\Omega)$, and $\left\|u_{\Gamma}\right\|_{L^{\infty}(\Omega)} \leq C$ for some constant $C>0$ independent of $\Gamma$; cf. [35]. This allows the use of the Lebesgue Dominated Convergence Theorem in the routine calculations of $\left.(d / d t)\right|_{t=0} I\left[u_{\Gamma}+t \eta\right]=0$ for any $\eta \in C_{\mathrm{c}}^{1}(\Omega)$ to obtain the equation in 2.23 . Since $C_{\mathrm{c}}^{1}(\Omega)$ is dense in $H_{0}^{1}(\Omega), 2.23$ holds true. The convexity of $B$ now imiplies that $u_{\Gamma}$ is the unique solution as desired.

The regularity of the soluton $\psi_{\Gamma}$ follows from the elliptic regularity theory $12,21,25,28,29$, 32, 39], with the same argument above for the regularity of the function $\hat{\phi}_{\Gamma, \infty}$; cf. (2.15) and (2.16). Note that the piecewise $C^{\infty}$ smoothness follows from a usual bootstrapping method.

(2) This part of the proof is the same as that given in [34].

\subsection{Electrostatic Free-Energy Functional of Ionic Concentrations}

We define

$$
\begin{aligned}
& \mathcal{X}=\left\{\left(c_{1}, \ldots, c_{M}\right) \in L^{1}\left(\Omega, \mathbb{R}^{M}\right): c_{j}=0 \text { a.e. } \Omega_{-} \text {for } j=1, \ldots, M \text { and } \sum_{j=1}^{M} q_{j} c_{j} \in H^{-1}(\Omega)\right\}, \\
& \mathcal{X}_{+}=\left\{\left(c_{1}, \ldots, c_{M}\right) \in \mathcal{X}: c_{j} \geq 0 \text { a.e. } \Omega_{+} \text {for } j=1, \ldots, M\right\} .
\end{aligned}
$$

Here, for any $g \in L^{1}(\Omega)$, we define $g \in L^{1}(\Omega) \cap H^{-1}(\Omega)$ by $(2.19)$. The space $\mathcal{X}$ is a Banach space equipped with the norm

$$
\|c\|_{\mathcal{X}}=\sum_{j=1}^{M}\left\|c_{j}\right\|_{L^{1}(\Omega)}+\left\|\sum_{j=1}^{M} q_{j} c_{j}\right\|_{H^{-1}(\Omega)} \quad \forall c=\left(c_{1}, \ldots, c_{M}\right) \in \mathcal{X} .
$$

Moreover, $\mathcal{X}_{+}$is a convex and closed subset of $\mathcal{X}$. For any $c=\left(c_{1}, \ldots, c_{M}\right) \in \mathcal{X}$, standard arguments (cf. 21, 22, 25, 40]) imply that there exists a unique weak solution $\psi$ to the boundary-value problem (1.4), defined by $\psi \in \hat{\phi}_{\mathrm{C}}+H^{1}(\Omega), \psi=\phi_{\infty}$ on $\partial \Omega$, and

$$
\int_{\Omega} \varepsilon_{\Gamma} \nabla \psi \cdot \nabla \eta d x=\sum_{i=1}^{N} Q_{i} \eta\left(x_{i}\right)+\int_{\Omega_{+}}\left(\sum_{j=1}^{M} q_{j} c_{j}\right) \eta d x \quad \forall \eta \in C_{\mathrm{c}}^{1}(\Omega),
$$


Equivalently, if $\hat{\phi} \in \hat{\phi}_{\mathrm{C}}+H^{1}(\Omega)$ satisfies 2.3 , then

$$
\int_{\Omega} \varepsilon_{\Gamma} \nabla(\psi-\hat{\phi}) \cdot \nabla \eta d x=\int_{\Omega_{+}}\left[\left(\varepsilon_{-}-\varepsilon_{+}\right) \nabla \hat{\phi} \cdot \nabla \eta+\left(\sum_{j=1}^{M} q_{j} c_{j}\right) \eta\right] d x \quad \forall \eta \in H_{0}^{1}(\Omega) .
$$

Clearly, $\psi-\hat{\phi}$ is harmonic in $\Omega_{-}$. Moreover, it follows from the definition of $\hat{\phi}_{\Gamma, \infty}$ (cf. (2.11) and $L_{\Gamma}$ (cf. (2.17) ) that

$$
\psi=\hat{\phi}_{\Gamma, \infty}+L_{\Gamma}\left(\sum_{j=1}^{M} q_{j} c_{j}\right)
$$

Since the function $s \mapsto s \log s(s \geq 0)$ is bounded below and $\Omega$ is bounded, $F_{\Gamma}[c]>-\infty$ for any $c \in \mathcal{X}_{+}$, where $F_{\Gamma}[c]$ is defined in (1.3).

Theorem 2.2. Let $\psi_{\Gamma}$ be the unique weak solution to the dielectric-boundary $P B$ equation (1.8). For each $j \in\{1, \ldots, M\}$, define $c_{\Gamma, j}: \Omega \rightarrow[0, \infty)$ by

$$
c_{\Gamma, j}(x)= \begin{cases}0 & \text { if } x \in \overline{\Omega_{-}}, \\ c_{j}^{\infty} e^{-\beta q_{j}\left[\psi_{\Gamma}(x)-\phi_{\Gamma, \infty}(x) / 2\right]} & \text { if } x \in \Omega_{+} .\end{cases}
$$

Then $c_{\Gamma}:=\left(c_{\Gamma, 1}, \ldots, c_{\Gamma, M}\right) \in \mathcal{X}_{+}$and $\psi_{\Gamma}$ is the electrostatic potential corresponding to $c_{\Gamma}$, i.e., the unique weak solution to (1.4) with $c_{j}$ replaced by $c_{\Gamma, j}(j=1, \ldots, M)$. Moreover, $c_{\Gamma}$ is the unique minimizer of the functional $F_{\Gamma}: \mathcal{X}_{+} \rightarrow(-\infty, \infty]$ defined in (1.3), and

$$
\begin{aligned}
F_{\Gamma}\left[c_{\Gamma}\right]=\frac{1}{2} & \sum_{i=1}^{N} Q_{i}\left(\psi_{\Gamma}-\hat{\phi}_{\mathrm{C}}\right)\left(x_{i}\right) \\
& +\int_{\Omega_{+}}\left[\frac{1}{2}\left(\psi_{\Gamma}-\phi_{\Gamma, \infty}\right) B^{\prime}\left(\psi_{\Gamma}-\frac{\phi_{\Gamma, \infty}}{2}\right)-B\left(\psi_{\Gamma}-\frac{\phi_{\Gamma, \infty}}{2}\right)\right] d x
\end{aligned}
$$

Proof. By the properties of $\psi_{\Gamma}$ (cf. Theorem 2.1) and $\phi_{\Gamma, \infty}$ (cf. (2.7)), we have $c_{\Gamma} \in \mathcal{X}_{+}$. If we replace $c_{j}$ in (1.4) by $c_{\Gamma, j}$ defined in (2.26) and note the definition of $B$ in (1.2), we get exactly the PB equation (1.8). Therefore, the unique solution $\psi_{\Gamma}$ to the boundary-value problem of the $\mathrm{PB}$ equation (1.8) is also the unique solution to the boundary-value problem of Poisson's equation 1.4 corresponding to $c_{\Gamma}$.

We now prove that $c_{\Gamma}$ is the unique minimizer of $F_{\Gamma}: \mathcal{X}_{+} \rightarrow(-\infty, \infty]$. To do so, we first re-write the functional $F_{\Gamma}$. Let $c=\left(c_{1}, \ldots, c_{M}\right) \in \mathcal{X}_{+}$and let $\psi \in \hat{\phi}_{\mathrm{C}}+H^{1}(\Omega)$ be the corresponding electrostatic potential, i.e., the weak solution to (1.4) defined in (2.24). Denote $f=\sum_{j=1}^{M} q_{j} c_{j}$. Since $f=0$ a.e. in $\Omega_{-}$, we have by the definition of $L_{\Gamma}($ cf. 2.17$)$ ) that $L_{\Gamma} f$ is harmonic in $\Omega_{-}$. Moreover,

$$
\sum_{i=1}^{N} Q_{i}\left(L_{\Gamma} f\right)\left(x_{i}\right)=\int_{\Omega_{+}}\left(\hat{\phi}_{\Gamma, \infty}-\phi_{\Gamma, \infty}\right) f d x
$$


cf. Lemma 3.2 in 34] (where $L /(4 \pi)$ and $G /(4 \pi)$ are our $L_{\Gamma}$ and $\hat{\phi}_{\Gamma, \infty}-\phi_{\Gamma, \infty}$ here, respectively). This, together with 2.25 and the fact that all $\psi-\hat{\phi}_{\mathrm{C}}, \hat{\phi}_{\Gamma, \infty}-\hat{\phi}$, and $L_{\Gamma} f$ are harmonic in $\Omega_{-}$, implies that

$$
\begin{aligned}
\sum_{i=1}^{N} Q_{i}\left(\psi-\hat{\phi}_{\mathrm{C}}\right)\left(x_{i}\right) & =\sum_{i=1}^{N} Q_{i}\left(L_{\Gamma} f\right)\left(x_{i}\right)+\sum_{i=1}^{N} Q_{i}\left(\hat{\phi}_{\Gamma, \infty}-\hat{\phi}_{\mathrm{C}}\right)\left(x_{i}\right) \\
& =\int_{\Omega_{+}}\left(\hat{\phi}_{\Gamma, \infty}-\phi_{\Gamma, \infty}\right)\left(\sum_{j=1}^{M} q_{j} c_{j}\right) d x+\sum_{i=1}^{N} Q_{i}\left(\hat{\phi}_{\Gamma, \infty}-\hat{\phi}_{\mathrm{C}}\right)\left(x_{i}\right) .
\end{aligned}
$$

With this and (2.25), we can rewrite $F_{\Gamma}[c](1.3)$ as

$$
F_{\Gamma}[c]=\int_{\Omega_{+}}\left[\frac{1}{2}\left(\sum_{j=1}^{M} q_{j} c_{j}\right) L_{\Gamma}\left(\sum_{j=1}^{M} q_{j} c_{j}\right)+\sum_{j=1}^{M}\left(\beta^{-1} c_{j} \log c_{j}+\alpha_{j} c_{j}\right)\right] d x+E_{0, \Gamma}
$$

where all $\alpha_{j}=\alpha_{j}(x)(j=1, \ldots, M)$ and $E_{0, \Gamma}$ are independent of $c$, given by

$$
\begin{aligned}
& \alpha_{j}(x)=q_{j}\left[\hat{\phi}_{\Gamma, \infty}(x)-\frac{1}{2} \phi_{\Gamma, \infty}(x)\right]+\beta^{-1}(3 \log \Lambda-1)-\mu_{j} \quad \forall x \in \Omega, j=1, \ldots, M, \\
& E_{0, \Gamma}=\frac{1}{2} \sum_{i=1}^{N} Q_{i}\left(\hat{\phi}_{\Gamma, \infty}-\hat{\phi}_{\mathrm{C}}\right)\left(x_{i}\right)+\beta^{-1}\left|\Omega_{+}\right| \sum_{j=1}^{M} c_{j}^{\infty} .
\end{aligned}
$$

Here and below, we denote by $|A|$ the Lebesgue measure of $A$ when no confusion arises.

We now compare $F_{\Gamma}[c]$ and $F_{\Gamma}\left[c_{\Gamma}\right]$. By Taylor's expansion, we have for any $s, t \in(0, \infty)$ that

$$
s \log s-t \log t=(1+\log t)(s-t)+\frac{1}{2 r}(s-t)^{2} \geq(1+\log t)(s-t),
$$

where $r$ is in between $s$ and $t$. Consequently, by (2.28) and the fact that $L_{\Gamma}$ is self-adjoint, we have

$$
\begin{aligned}
F_{\Gamma}[c]-F_{\Gamma}\left[c_{\Gamma}\right]= & \int_{\Omega} \frac{1}{2}\left(\sum_{j=1}^{M} q_{j}\left(c_{j}-c_{\Gamma, j}\right)\right) L_{\Gamma}\left(\sum_{j=1}^{M} q_{j}\left(c_{j}-c_{\Gamma, j}\right)\right) d x \\
& +\int_{\Omega}\left(\sum_{j=1}^{M} q_{j}\left(c_{j}-c_{\Gamma, j}\right)\right) L_{\Gamma}\left(\sum_{k=1}^{M} q_{k} c_{\Gamma, k}\right) d x \\
& +\beta^{-1} \sum_{j=1}^{M} \int_{\Omega}\left(c_{j} \log c_{j}-c_{\Gamma, j} \log c_{\Gamma, j}\right) d x+\sum_{j=1}^{M} \int_{\Omega}\left(c_{j}-c_{\Gamma, j}\right) \alpha_{j} d x \\
\geq & \sum_{j=1}^{M} \int_{\Omega}\left(c_{j}-c_{\Gamma, j}\right)\left[q_{j} L_{\Gamma}\left(\sum_{k=1}^{M} q_{k} c_{\Gamma, k}\right)+\beta^{-1}\left(1+\log c_{\Gamma, j}\right)+\alpha_{j}\right] d x .
\end{aligned}
$$


It follows from the fact that $c_{j}^{\infty}=\Lambda^{-3} e^{\beta \mu_{j}}$ (cf. the assumption (A2)), 2.25), 2.26), and 2.29 ) that the quantity inside the brackets in the above integral vanishes. Thus, $F[c] \geq \bar{F}\left[c_{\Gamma}\right]$. Hence, $c_{\Gamma}$ is a minimizer of $F_{\Gamma}: \mathcal{X}_{+} \rightarrow(-\infty, \infty]$. Since $F_{\Gamma}$ is convex, and in particular, $s \mapsto s \log s$ is strictly convex on $(0, \infty)$, the minimizer of $F_{\Gamma}$ is unique; cf. [34].

Finally, we obtain (2.27) from (1.3) with $\psi_{\Gamma}$ and $c_{\Gamma}$ replacing $\psi$ and $c$, respectively, 1.2), and 2.26 .

\section{Dielectric Boundary Force}

\subsection{Electrostatic Free Energy of a Dielectric Boundary}

Given any dielectric boundary $\Gamma$, we denote by

$$
E[\Gamma]=\min _{c \in \mathcal{X}_{+}} F_{\Gamma}[c]
$$

the minimum electrostatic free energy given in Theorem 2.2 (cf. (2.27)). We reformulate $E[\Gamma]$ to convert the discrete part of the energy into volume integrals that will be useful when we calculate the variation of $E[\Gamma]$ with respect to the boundary variation of $\Gamma$.

Lemma 3.1. Let $\Gamma$ be a dielectric boundary satisfying the part of the assumption A1 on $\Gamma$ in Subsection 2.1. Let $\psi_{\Gamma} \in \hat{\phi}_{\mathrm{C}}+H^{1}(\Omega)$ be the corresponding solution to the boundary-value problem of $P B$ equation (1.8). We have

$$
\begin{aligned}
E[\Gamma]=- & \int_{\Omega} \frac{\varepsilon_{\Gamma}}{2}\left|\nabla\left(\psi_{\Gamma}-\hat{\phi}_{\Gamma, \infty}\right)\right|^{2} d x-\int_{\Omega_{+}} B\left(\psi_{\Gamma}-\frac{\phi_{\Gamma, \infty}}{2}\right) d x \\
& +\frac{\varepsilon_{-}-\varepsilon_{+}}{2} \int_{\Omega_{+}} \nabla \hat{\phi}_{\Gamma, \infty} \cdot \nabla \hat{\phi}_{0} d x+\frac{1}{2} \sum_{i=1}^{N} Q_{i}\left(\hat{\phi}_{\infty}-\hat{\phi}_{\mathrm{C}}\right)\left(x_{i}\right),
\end{aligned}
$$

where all the functions $\hat{\phi}_{\mathrm{C}}, \hat{\phi}_{0}, \hat{\phi}_{\infty}, \phi_{\Gamma, \infty}$, and $\hat{\phi}_{\Gamma, \infty}$ are defined in Subsection 2.1.

Proof. We first prove an elementary identity. Let $u \in C^{2}\left(\Omega_{-}\right) \cap C^{1}\left(\overline{\Omega_{-}}\right)$be such that $\Delta u=0$ in $\Omega_{-}$. Let $v \in \hat{\phi}_{\mathrm{C}}+H^{1}\left(\Omega_{-}\right) \cap C\left(\overline{\Omega_{-}}\right)$, in particular, $v=\hat{\phi}_{\mathrm{C}}, \hat{\phi}_{0}, \hat{\phi}_{\infty}$, or $\hat{\phi}_{\Gamma, \infty}$ (restricted onto $\left.\Omega_{-}\right)$. Denote $B_{\alpha}=\cup_{i=1}^{N} B\left(x_{i}, \alpha\right)$ for $0<\alpha \ll 1$ and $\nu$ the unit normal at $\partial B(\alpha)=\cup_{i=1}^{N} \partial B\left(x_{i}, \alpha\right)$, pointing toward $x_{i}(i=1, \ldots, N)$. Since the unit normal $n$ at $\Gamma$ points from $\Omega_{-}$to $\Omega_{+}$, and since $v=\hat{\phi}_{\mathrm{C}}+\hat{v}$ for some $\hat{v} \in H^{1}\left(\Omega_{-}\right) \cap C\left(\overline{\Omega_{-}}\right)$and $\hat{\phi}_{\mathrm{C}}$ is given in $(1.6)$, we have

$$
\begin{aligned}
\int_{\Omega_{-}} \nabla u \cdot \nabla v d x & =\lim _{\alpha \rightarrow 0^{+}} \int_{\Omega_{-} \backslash B_{\alpha}} \nabla u \cdot \nabla v d x \\
& =\int_{\Gamma} \partial_{n} u v d S+\lim _{\alpha \rightarrow 0^{+}} \sum_{i=1}^{N} \int_{\partial B\left(x_{i}, \alpha\right)} \partial_{\nu} u v d S \\
& =\int_{\Gamma} \partial_{n} u v d S .
\end{aligned}
$$


Denoting now $W=(1 / 2) \sum_{i=1}^{N} Q_{i}\left(\hat{\phi}_{\infty}-\hat{\phi}_{\mathrm{C}}\right)\left(x_{i}\right)$, we have by 3.1) and 2.27) that

$$
\begin{aligned}
E[\Gamma]=\frac{1}{2} & \sum_{i=1}^{N} Q_{i}\left(\hat{\phi}_{\Gamma, \infty}-\hat{\phi}_{\infty}\right)\left(x_{i}\right)+\frac{1}{2} \sum_{i=1}^{N} Q_{i}\left(\psi_{\Gamma}-\hat{\phi}_{\Gamma, \infty}\right)\left(x_{i}\right) \\
& +\int_{\Omega_{+}}\left[\frac{1}{2}\left(\psi_{\Gamma}-\phi_{\Gamma, \infty}\right) B^{\prime}\left(\psi_{\Gamma}-\frac{\phi_{\Gamma, \infty}}{2}\right)-B\left(\psi_{\Gamma}-\frac{\phi_{\Gamma, \infty}}{2}\right)\right] d x+W .
\end{aligned}
$$

We first consider the first term in (3.4). Note that the unit vector $n$ normal to $\Gamma$ points from $\Omega_{-}$to $\Omega_{+}$. We have by Green's formula that

$$
\begin{aligned}
& \frac{1}{2} \sum_{i=1}^{N} Q_{i}\left(\hat{\phi}_{\Gamma, \infty}-\hat{\phi}_{\infty}\right)\left(x_{i}\right) \\
& =\int_{\Omega} \frac{\varepsilon_{-}}{2} \nabla \hat{\phi}_{0} \cdot \nabla\left(\hat{\phi}_{\Gamma, \infty}-\hat{\phi}_{\infty}\right) d x \quad\left[\text { by } 2.3 \text { with } \hat{\phi}=\hat{\phi}_{0} \text { and } \eta=\hat{\phi}_{\Gamma, \infty}-\hat{\phi}_{\infty}\right] \\
& =\int_{\Omega_{-}} \frac{\varepsilon_{-}}{2} \nabla \hat{\phi}_{0} \cdot \nabla\left(\hat{\phi}_{\Gamma, \infty}-\hat{\phi}_{\infty}\right) d x+\int_{\Omega_{+}} \frac{\varepsilon_{-}}{2} \nabla \hat{\phi}_{0} \cdot \nabla\left(\hat{\phi}_{\Gamma, \infty}-\hat{\phi}_{\infty}\right) d x \\
& =\int_{\Gamma} \frac{\varepsilon_{-}}{2} \hat{\phi}_{0} \partial_{n}\left(\hat{\phi}_{\Gamma, \infty}^{-}-\hat{\phi}_{\infty}\right) d S+\int_{\Omega_{+}} \frac{\varepsilon_{-}}{2} \nabla \hat{\phi}_{0} \cdot \nabla\left(\hat{\phi}_{\Gamma, \infty}-\hat{\phi}_{\infty}\right) d x \quad \text { [by (3.3)] } \\
& \left.=\int_{\Gamma} \frac{\varepsilon_{+}}{2} \hat{\phi}_{0} \partial_{n} \hat{\phi}_{\Gamma, \infty}^{+} d S-\int_{\Gamma} \frac{\varepsilon_{-}}{2} \hat{\phi}_{0} \partial_{n} \hat{\phi}_{\infty} d S \quad \text { [by 2.14] }\right] \\
& +\int_{\Omega_{+}} \frac{\varepsilon_{-}}{2} \nabla \hat{\phi}_{0} \cdot \nabla\left(\hat{\phi}_{\Gamma, \infty}-\hat{\phi}_{\infty}\right) d x \\
& =-\int_{\partial \Omega_{+}} \frac{\varepsilon_{+}}{2} \hat{\phi}_{0} \partial_{n} \hat{\phi}_{\Gamma, \infty} d S+\int_{\partial \Omega_{+}} \frac{\varepsilon_{-}}{2} \hat{\phi}_{0} \partial_{n} \hat{\phi}_{\infty} d S \quad\left[\text { since } \hat{\phi}_{0}=0 \text { on } \partial \Omega\right] \\
& +\int_{\Omega_{+}} \frac{\varepsilon_{-}}{2} \nabla \hat{\phi}_{0} \cdot \nabla\left(\hat{\phi}_{\Gamma, \infty}-\hat{\phi}_{\infty}\right) d x \\
& =-\int_{\Omega_{+}} \frac{\varepsilon_{+}}{2} \nabla \hat{\phi}_{0} \cdot \nabla \hat{\phi}_{\Gamma, \infty} d x+\int_{\Omega_{+}} \frac{\varepsilon_{-}}{2} \nabla \hat{\phi}_{0} \cdot \nabla \hat{\phi}_{\infty} d x \\
& +\int_{\Omega_{+}} \frac{\varepsilon_{-}}{2} \nabla \hat{\phi}_{0} \cdot \nabla\left(\hat{\phi}_{\Gamma, \infty}-\hat{\phi}_{\infty}\right) d x \\
& =\frac{\varepsilon_{-}-\varepsilon_{+}}{2} \int_{\Omega_{+}} \nabla \hat{\phi}_{\Gamma, \infty} \cdot \nabla \hat{\phi}_{0} d x .
\end{aligned}
$$

Considering now the second and third terms in $(3.4)$, we have

$$
\begin{aligned}
& \frac{1}{2} \sum_{i=1}^{N} Q_{i}\left(\psi_{\Gamma}-\hat{\phi}_{\Gamma, \infty}\right)\left(x_{i}\right) \\
& \quad+\int_{\Omega_{+}}\left[\frac{1}{2}\left(\psi_{\Gamma}-\phi_{\Gamma, \infty}\right) B^{\prime}\left(\psi_{\Gamma}-\frac{\phi_{\Gamma, \infty}}{2}\right)-B\left(\psi_{\Gamma}-\frac{\phi_{\Gamma, \infty}}{2}\right)\right] d x
\end{aligned}
$$




$$
\begin{aligned}
& =\sum_{i=1}^{N} Q_{i}\left(\psi_{\Gamma}-\hat{\phi}_{\Gamma, \infty}\right)\left(x_{i}\right)-\frac{1}{2} \sum_{i=1}^{N} Q_{i}\left(\psi_{\Gamma}-\hat{\phi}_{\Gamma, \infty}\right)\left(x_{i}\right) \\
& +\int_{\Omega_{+}}\left[\frac{1}{2}\left(\psi_{\Gamma}-\phi_{\Gamma, \infty}\right) B^{\prime}\left(\psi_{\Gamma}-\frac{\phi_{\Gamma, \infty}}{2}\right)-B\left(\psi_{\Gamma}-\frac{\phi_{\Gamma, \infty}}{2}\right)\right] d x \\
& =\int_{\Omega} \varepsilon_{\Gamma} \nabla \hat{\phi}_{\Gamma, \infty} \cdot \nabla\left(\psi_{\Gamma}-\hat{\phi}_{\Gamma, \infty}\right) d x \quad[\text { by 2.11] }] \\
& -\frac{1}{2} \int_{\Omega}\left[\varepsilon_{\Gamma} \nabla \psi_{\Gamma} \cdot \nabla\left(\psi_{\Gamma}-\hat{\phi}_{\Gamma, \infty}\right)+\chi_{+} B^{\prime}\left(\psi_{\Gamma}-\frac{\phi_{\Gamma, \infty}}{2}\right)\left(\psi_{\Gamma}-\hat{\phi}_{\Gamma, \infty}\right)\right] \\
& +\int_{\Omega_{+}}\left[\frac{1}{2}\left(\psi_{\Gamma}-\phi_{\Gamma, \infty}\right) B^{\prime}\left(\psi_{\Gamma}-\frac{\phi_{\Gamma, \infty}}{2}\right)-B\left(\psi_{\Gamma}-\frac{\phi_{\Gamma, \infty}}{2}\right)\right] d x \\
& =-\int_{\Omega} \frac{\varepsilon_{\Gamma}}{2}\left|\nabla\left(\psi_{\Gamma}-\hat{\phi}_{\Gamma, \infty}\right)\right|^{2} d x-\int_{\Omega_{+}} B\left(\psi_{\Gamma}-\frac{\phi_{\Gamma, \infty}}{2}\right) d x \\
& +\int_{\Omega} \frac{\varepsilon_{\Gamma}}{2} \nabla\left(\psi_{\Gamma}-\hat{\phi}_{\Gamma, \infty}\right) \cdot \nabla \hat{\phi}_{\Gamma, \infty} d x+\int_{\Omega_{+}} \frac{1}{2}\left(\hat{\phi}_{\Gamma, \infty}-\phi_{\Gamma, \infty}\right) B^{\prime}\left(\psi_{\Gamma}-\frac{\phi_{\Gamma, \infty}}{2}\right) d x \\
& =-\int_{\Omega} \frac{\varepsilon_{\Gamma}}{2}\left|\nabla\left(\psi_{\Gamma}-\hat{\phi}_{\Gamma, \infty}\right)\right|^{2} d x-\int_{\Omega_{+}} B\left(\psi_{\Gamma}-\frac{\phi_{\Gamma, \infty}}{2}\right) d x \\
& +\int_{\Omega} \frac{\varepsilon_{\Gamma}}{2} \nabla\left(\psi_{\Gamma}-\hat{\phi}_{\Gamma, \infty}\right) \cdot \nabla \hat{\phi}_{\Gamma, \infty} d x \\
& +\int_{\Omega_{+}} \frac{\varepsilon_{+}}{2}\left(\hat{\phi}_{\Gamma, \infty}-\phi_{\Gamma, \infty}\right) \Delta\left(\psi_{\Gamma}-\hat{\phi}_{\Gamma, \infty}\right) d x \quad \text { [by 2.22 and 2.13] } \\
& =-\int_{\Omega} \frac{\varepsilon_{\Gamma}}{2}\left|\nabla\left(\psi_{\Gamma}-\hat{\phi}_{\Gamma, \infty}\right)\right|^{2} d x-\int_{\Omega_{+}} B\left(\psi_{\Gamma}-\frac{\phi_{\Gamma, \infty}}{2}\right) d x \\
& +\int_{\Omega} \frac{\varepsilon_{\Gamma}}{2} \nabla\left(\psi_{\Gamma}-\hat{\phi}_{\Gamma, \infty}\right) \cdot \nabla\left(\hat{\phi}_{\Gamma, \infty}-\phi_{\Gamma, \infty}\right) d x \quad[\text { by 2.5 }] \\
& -\int_{\Omega_{+}} \frac{\varepsilon_{+}}{2} \nabla\left(\hat{\phi}_{\Gamma, \infty}-\phi_{\Gamma, \infty}\right) \cdot \nabla\left(\psi_{\Gamma}-\hat{\phi}_{\Gamma, \infty}\right) d x \\
& -\int_{\Gamma} \frac{\varepsilon_{+}}{2} \partial_{n}\left(\psi_{\Gamma}^{+}-\hat{\phi}_{\Gamma, \infty}^{+}\right)\left(\hat{\phi}_{\Gamma, \infty}-\phi_{\Gamma, \infty}\right) d S \quad\left[\text { since } \hat{\phi}_{\Gamma, \infty}-\phi_{\Gamma, \infty}=0 \text { on } \partial \Omega\right] \\
& =-\int_{\Omega} \frac{\varepsilon_{\Gamma}}{2}\left|\nabla\left(\psi_{\Gamma}-\hat{\phi}_{\Gamma, \infty}\right)\right|^{2} d x-\int_{\Omega_{+}} B\left(\psi_{\Gamma}-\frac{\phi_{\Gamma, \infty}}{2}\right) d x \\
& +\int_{\Omega_{-}} \frac{\varepsilon_{-}}{2} \nabla\left(\psi_{\Gamma}-\hat{\phi}_{\Gamma, \infty}\right) \cdot \nabla\left(\hat{\phi}_{\Gamma, \infty}-\phi_{\Gamma, \infty}\right) d x \\
& -\int_{\Gamma} \frac{\varepsilon_{-}}{2} \partial_{n}\left(\psi_{\Gamma}^{-}-\hat{\phi}_{\Gamma, \infty}^{-}\right)\left(\hat{\phi}_{\Gamma, \infty}-\phi_{\Gamma, \infty}\right) d S \quad \text { [by 2.22) with } \psi=\psi_{\Gamma} \text { and (2.14)] } \\
& =-\int_{\Omega} \frac{\varepsilon_{\Gamma}}{2}\left|\nabla\left(\psi_{\Gamma}-\hat{\phi}_{\Gamma, \infty}\right)\right|^{2} d x-\int_{\Omega_{+}} B\left(\psi_{\Gamma}-\frac{\phi_{\Gamma, \infty}}{2}\right) d x . \quad[\text { by (3.3)] }
\end{aligned}
$$

Now (3.2) follows directly from (3.4) and the above two expressions. 
We define $G_{\Gamma}: \hat{\phi}_{\Gamma, \infty}+H_{0}^{1}(\Omega) \rightarrow \mathbb{R} \cup\{-\infty\}$ by

$$
G_{\Gamma}[\psi]=-\int_{\Omega} \frac{\varepsilon_{\Gamma}}{2}\left|\nabla\left(\psi-\hat{\phi}_{\Gamma, \infty}\right)\right|^{2} d x-\int_{\Omega_{+}} B\left(\psi-\frac{\phi_{\Gamma, \infty}}{2}\right) d x+g_{\Gamma, \infty}
$$

where

$$
g_{\Gamma, \infty}=\frac{\varepsilon_{-}-\varepsilon_{+}}{2} \int_{\Omega_{+}} \nabla \hat{\phi}_{\Gamma, \infty} \cdot \nabla \hat{\phi}_{0} d x+\frac{1}{2} \sum_{i=1}^{N} Q_{i}\left(\hat{\phi}_{\infty}-\hat{\phi}_{\mathrm{C}}\right)\left(x_{i}\right) .
$$

We shall call $G_{\Gamma}$ the PB energy functional. Note that by Lemma 3.1, $E[\Gamma]=G_{\Gamma}\left[\psi_{\Gamma}\right]$. In fact, we have the following variational principle for the PB energy functional.

Theorem 3.1. The Euler-Lagrange equation of the PB energy functional $G_{\Gamma}: \hat{\phi}_{\Gamma, \infty}+$ $H_{0}^{1}(\Omega) \rightarrow \mathbb{R} \cup\{-\infty\}$ is exactly the dielectric-boundary PB equation. Moreover, the functional $G_{\Gamma}[\cdot]$ is uniquely maximized over $\hat{\phi}_{\Gamma, \infty}+H_{0}^{1}(\Omega)$ by the solution $\psi_{\Gamma}$ to the boundary-value problem of the $P B$ equation (1.8), and the maximum value is exactly $E[\Gamma]$.

Proof. Direct calculations verify that the Euler-Lagrange equation for the PB energy functional $G_{\Gamma}[\cdot]$ is indeed the dielectric-boundary PB equation; cf. Definition 2.1. The existence of a unique maximizer can be proved exactly the same way as in the proof of Theorem 2.1. These, together with Lemma 3.1, imply that the maximum value of the free energy is $G_{\Gamma}\left[\psi_{\Gamma}\right]=E[\Gamma]$.

We remark that the $\mathrm{PB}$ functional $G_{\Gamma}[\cdot]$ is maximized, not minimized, among all the admissible electrostatic potentials. In general, for a charged system occupying a region $D \subseteq$ $\mathbb{R}^{3}$ with the dielectric coefficient $\varepsilon$ and charge density $\rho \in L^{2}(D)$, the commonly used energy functional of electrostatic potentials $\psi$ is given by

$$
\psi \mapsto \int_{D}\left(-\frac{\varepsilon}{2}|\nabla \psi|^{2}+\rho \psi\right) d x
$$

With suitable boundary conditions, this functional is maximized by a unique electrostatic potential. This maximizer is exactly the solution to Poisson's equation, which is the EulerLagrange equation of this functional. Moreover, the maximum value of the functional is exactly the electrostatic energy corresponding to the potential determined by Poisson's equation. See [7] for more related discussions.

\subsection{Definition and Formula of the Dielectric Boundary Force}

Let $\Gamma$ be a dielectric boundary as given in the assumption A1 in Subsection 2.1. Let $\phi: \mathbb{R}^{3} \rightarrow$ $\mathbb{R}$ be the signed distance function to $\Gamma$, negative in $\Omega_{-}$(inside $\Gamma$ ) and positive in $\mathbb{R}^{3} \backslash \overline{\Omega_{-}}$ (outside $\Gamma$ ). Then, $n=\nabla \phi$ is exactly the unit normal along $\Gamma$, pointing from $\Omega_{-}$to $\Omega_{+}$. Since $\Gamma$ is assumed to be of the class $C^{3}$, there exists $d_{0}>0$ with

$$
d_{0}<\frac{1}{2} \min \left(\operatorname{dist}(\Gamma, \partial \Omega), \min _{1 \leq i \leq N} \operatorname{dist}\left(x_{i}, \Gamma\right)\right)
$$


such that the signed distance function $\phi$ is a $C^{3}$-function and $\nabla \phi \neq 0$ in the neighborhood

$$
\mathcal{N}_{0}(\Gamma)=\left\{x \in \Omega: \operatorname{dist}(x, \Gamma)<d_{0}\right\}
$$

in $\Omega$ of $\Gamma$; cf. 25] (Section 14.6) and [31]. Define

$$
\mathcal{V}=\left\{V \in C_{\mathrm{c}}^{2}\left(\mathbb{R}^{3}, \mathbb{R}^{3}\right): \operatorname{supp}(V) \subset \mathcal{N}_{0}(\Gamma)\right\}
$$

Let $V \in \mathcal{V}$. For any $X \in \mathbb{R}^{3}$, let $x=x(t, X)$ be the unique solution to the initial-value problem

$$
\dot{x}=V(x) \quad(t \in \mathbb{R}) \quad \text { and } \quad x(0, X)=X,
$$

where a dot denotes the derivative with respect to $t$. Define $T_{t}(X)=x(t, X)$ for any $X \in \mathbb{R}^{3}$ and any $t \in \mathbb{R}$. Then, $\left\{T_{t}\right\}_{t \in \mathbb{R}}$ is a family of diffeomorphisms and $C^{2}$-maps from $\mathbb{R}^{3}$ to $\mathbb{R}^{3}$ with $T_{0}=I$ the identity map and $T_{-t}=T_{t}^{-1}$ for any $t \in \mathbb{R}$.

Let $t \in \mathbb{R}$. Since $\operatorname{supp}(V) \subset \mathcal{N}_{0}(\Gamma) \subset \Omega$, we have $T_{t}(\Omega)=\Omega$ and $T_{t}(\partial \Omega)=\partial \Omega$. Clearly, $T_{t}\left(\Omega_{-}\right) \subset \Omega$ and $T_{t}\left(\Omega_{+}\right)=\Omega \backslash \overline{T_{t}\left(\Omega_{-}\right)}$. Moreover, $\Gamma_{t}:=T_{t}(\Gamma)=\partial T_{t}\left(\Omega_{-}\right)=\overline{T_{t}\left(\Omega_{-}\right)} \cap \overline{T_{t}\left(\Omega_{+}\right)}$is of class $C^{2}$. Note that $x_{i} \in T_{t}\left(\Omega_{-}\right)$and $T_{t}\left(x_{i}\right)=x_{i}$ for all $i=1, \ldots, N$. Analogous to $(1.5), \varepsilon_{\Gamma_{t}}$ is defined correspondingly with respect to $T_{t}\left(\Omega_{-}\right)$and $T_{t}\left(\Omega_{+}\right)$. We shall denote $\Gamma_{t}=\Gamma_{t}(V)$ to indicate the dependence of $\Gamma_{t}$ on $V \in \mathcal{V}$. For each $t \in \mathbb{R}$, the electrostatic free energy $E\left[\Gamma_{t}(V)\right]$ is defined in (3.1) (cf. also (3.2) with $\Gamma_{t}=\Gamma_{t}(V)$ replacing $\Gamma$.

Definition 3.1. Let $V \in \mathcal{V}$. The first variation of $E[\Gamma]$ with respect to the perturbation of $\Gamma$ defined by $V$ is

$$
\delta_{\Gamma, V} E[\Gamma]=\left.\frac{d}{d t} E\left[\Gamma_{t}(V)\right]\right|_{t=0}=\lim _{t \rightarrow 0^{+}} \frac{E\left[\Gamma_{t}(V)\right]-E[\Gamma]}{t}
$$

if the limit exists.

We recall that the tangential gradient along a dielectric boundary $\Gamma$ is given by $\nabla_{\Gamma}=$ $(I-n \otimes n) \nabla$, where $I$ is the identity matrix. The following theorem provides an explicit formula of the first variation $\delta_{\Gamma, V} E[\Gamma]$, and its proof is given in Section 5 ;

Theorem 3.2. Let $\Gamma$ be a given dielectric boundary as described in the assumption $A 1$ in Subsection 2.1. Let $\psi_{\Gamma} \in \hat{\phi}_{\Gamma, \infty}+H_{0}^{1}(\Omega)$ be the unique weak solution to the boundary-value problem of the dielectric-boundary $P B$ equation (1.8). Then, for any $V \in \mathcal{V}$, the first variation $\delta_{\Gamma, V} E[\Gamma]$ exists, and is given by

$$
\delta_{\Gamma, V} E[\Gamma]=\int_{\Gamma} q_{\Gamma}(V \cdot n) d S
$$

where

$$
\begin{aligned}
q_{\Gamma}= & -\frac{1}{2}\left(\frac{1}{\varepsilon_{+}}-\frac{1}{\varepsilon_{-}}\right)\left(\left|\varepsilon_{\Gamma} \partial_{n} \psi_{\Gamma}\right|^{2}-\varepsilon_{\Gamma} \partial_{n} \psi_{\Gamma} \varepsilon_{\Gamma} \partial_{n} \phi_{\Gamma, \infty}\right) \\
& +\frac{\varepsilon_{+}-\varepsilon_{-}}{2}\left(\left|\nabla_{\Gamma} \psi_{\Gamma}\right|^{2}-\nabla_{\Gamma} \psi_{\Gamma} \cdot \nabla_{\Gamma} \phi_{\Gamma, \infty}\right)+B\left(\psi_{\Gamma}-\frac{\phi_{\Gamma, \infty}}{2}\right) .
\end{aligned}
$$

We identify $q_{\Gamma}$ in $(3.8)$ as the first variation of $E[\Gamma]$ and denote it as $q_{\Gamma}=\delta_{\Gamma} E[\Gamma]$. We call $-\delta_{\Gamma} E[\Gamma]$ the (normal component of the) dielectric boundary force. 


\section{Some Lemmas: The Calculus of Boundary Variations}

\subsection{Properties of the Transformation $T_{t}$}

We first recall some properties of the family of transformations $T_{t}: \mathbb{R}^{3} \rightarrow \mathbb{R}^{3}(t \in \mathbb{R})$ defined by 3.7 in Subsection 3.2 above via a vector field $V \in C_{\mathrm{c}}^{2}\left(\mathbb{R}^{3}, \mathbb{R}^{3}\right)$. These properties hold true if we change $\mathbb{R}^{3}$ to $\mathbb{R}^{d}$ with a general dimension $d \geq 2$. They can be proved by direct calculations; cf. [18] (Section 4 of Chapter 9).

(1) Let $X \in \mathbb{R}^{3}$ and $t \in \mathbb{R}$. Let $\nabla T_{t}(X)$ be the gradient matrix of $T_{t}$ at $X$ with its entries $\left(\nabla T_{t}(X)\right)_{i j}=\partial_{j} T_{t}^{i}(X)(i, j=1,2,3)$, where $T_{t}^{i}$ is the $i$ th component of $T_{t}$. Let

$$
J_{t}(X)=\operatorname{det} \nabla T_{t}(X) .
$$

Then for each $X \in \mathbb{R}^{3}$ the function $t \mapsto J_{t}(X)$ is a $C^{2}$-function and

$$
\frac{d J_{t}}{d t}=\left((\nabla \cdot V) \circ T_{t}\right) J_{t}
$$

where $\circ$ denotes the composition of functions or maps. Clearly, $\nabla T_{0}=I$, the identity matrix, and $J_{0}=1$. Moreover,

$$
J_{t}(X)=1+t(\nabla \cdot V)(X)+H(t, X) t^{2} \quad \forall t \in \mathbb{R} \quad \forall X \in \mathbb{R}^{3},
$$

where $H(t, X)$ satisfies

$$
\sup \left\{|H(t, X)|: t \in \mathbb{R}, X \in \mathbb{R}^{3}\right\}<\infty,
$$

since $V$ is compactly supported.

(2) For each $t \in \mathbb{R}$, we define $A_{V}(t): \mathbb{R}^{3} \rightarrow \mathbb{R}$ by

$$
A_{V}(t)(X)=J_{t}(X)\left(\nabla T_{t}(X)\right)^{-1}\left(\nabla T_{t}(X)\right)^{-T},
$$

where a superscript $T$ denotes the matrix transpose. Clearly, $A(t) \in C^{1}\left(\mathbb{R}^{3}, \mathbb{R}^{3 \times 3}\right)$, and the $t$-derivative of $A_{V}(t)$ at each point in $\mathbb{R}^{3}$ is

$$
\begin{gathered}
A_{V}^{\prime}(t)=\left[\left((\nabla \cdot V) \circ T_{t}\right)-\left(\nabla T_{t}\right)^{-1}\left((\nabla V) \circ T_{t}\right) \nabla T_{t}\right. \\
\left.-\left(\nabla T_{t}\right)^{-1}\left((\nabla V) \circ T_{t}\right)^{T}\left(\nabla T_{t}\right)\right] A_{V}(t) .
\end{gathered}
$$

In particular

$$
A_{V}^{\prime}(0)=(\nabla \cdot V) I-\nabla V-(\nabla V)^{T}
$$

Moreover,

$$
A_{V}(t)(X)=I+t A_{V}^{\prime}(0)(X)+K(t, X) t^{2} \quad \forall t \in \mathbb{R} \quad \forall X \in \mathbb{R}^{3},
$$

where $K(t, X)$ satisfies

$$
\sup \left\{|K(t, X)|: t \in \mathbb{R}, X \in \mathbb{R}^{3}\right\}<\infty .
$$


(3) For any $u \in L^{2}(\Omega)$ and $t \in \mathbb{R}, u \circ T_{t} \in L^{2}(\Omega)$ and $u \circ T_{t}^{-1} \in L^{2}(\Omega)$. Moreover,

$$
\lim _{t \rightarrow 0} u \circ T_{t}=u \quad \text { and } \quad \lim _{t \rightarrow 0} u \circ T_{t}^{-1}=u \quad \text { in } L^{2}(\Omega) .
$$

For any $u \in H^{1}(\Omega)$ and $t \in \mathbb{R}, u \circ T_{t} \in H^{1}(\Omega)$ and $u \circ T_{t}^{-1} \in H^{1}(\Omega)$. Moreover,

$$
\begin{aligned}
& \nabla\left(u \circ T_{t}^{-1}\right)=\left(\nabla T_{t}^{-1}\right)^{T}\left(\nabla u \circ T_{t}^{-1}\right) \quad \text { and } \quad \nabla\left(u \circ T_{t}\right)=\left(\nabla T_{t}\right)^{T}\left(\nabla u \circ T_{t}\right), \\
& \lim _{t \rightarrow 0} u \circ T_{t}=u \quad \text { and } \quad \lim _{t \rightarrow 0} u \circ T_{t}^{-1}=u \quad \text { in } H^{1}(\Omega) .
\end{aligned}
$$

If $u \in H^{2}(\Omega)$, then

$$
\lim _{t \rightarrow 0}\left\|\frac{u \circ T_{t}-u}{t}-\nabla u \cdot V\right\|_{H^{1}(\Omega)}=0
$$

\subsection{Tangential Force}

This is a geometrical property on the effect of tangential component of a velocity vector field to the motion of an interface. We shall state and prove it for a general $d$-dimensional space $\mathbb{R}^{d}$ with $d \geq 2$. We assume that $\Gamma$ is a $C^{3}$, closed, hypersurface in $\mathbb{R}^{d}$. We denote as before the interior and exterior of $\Gamma$ by $\Omega_{-}$and $\Omega_{+}$, respectively. We also denote by $n$ the unit vector normal to the surface $\Gamma$ at a point on $\Gamma$, pointing from the interior to exterior of $\Gamma$. We assume that $V \in C_{\mathrm{c}}^{2}\left(\mathbb{R}^{d}, \mathbb{R}^{d}\right)$ and define the transformation $T_{t}: \mathbb{R}^{d} \rightarrow \mathbb{R}^{d}(t \in \mathbb{R})$ by $T_{t}(X)=x(t, X)$ for any $X \in \mathbb{R}^{d}$ and $t \in \mathbb{R}$, where $x=x(t, X)$ is the unique solution to the initial-value problem (3.7).

Lemma 4.1. If $V \cdot n=0$ on $\Gamma$, then there exist $t_{0}>0$ and $C>0$, depending on $\Gamma$ and $V$, such that

$$
\begin{aligned}
& \operatorname{dist}\left(T_{t}(X), \Gamma\right) \leq C t^{2} \quad \forall X \in \Gamma \forall t \in \mathbb{R} \quad \text { with }|t| \leq t_{0}, \\
& \left|\left\{x \in \mathbb{R}^{d}: \chi_{T_{t}\left(\Omega_{\mathrm{s}}\right)}(x) \neq \chi_{\Omega_{\mathrm{s}}}(x)\right\}\right| \leq C t^{2} \quad \text { if }|t| \leq t_{0}, \quad \mathrm{~s}=- \text { or }+.
\end{aligned}
$$

Proof. We first prove (4.13). Since $\Gamma$ is of $C^{3}$, there exist finitely many open balls in $\mathbb{R}^{d}$ such that their union covers $\Gamma$ and that the intersection of $\Gamma$ with each of such open balls is the graph of a $C^{3}$ function in a local coordinate system. Let us fix one of such open balls, $\mathcal{B}$, and assume without loss of generality that the corresponding $C^{3}$-function is given by $h: \prod_{j=1}^{d-1}\left(a_{j}-\delta, b_{j}+\delta\right) \rightarrow \mathbb{R}$ for some $a_{j}, b_{j} \in \mathbb{R}$ with $a_{j}<b_{j}(j=1, \ldots, d-1)$ and $\delta>0$, where $\mathcal{B} \cap \Gamma$ is the graph of $h$ on $\prod_{j=1}^{d-1}\left(a_{j}-\delta, b_{j}+\delta\right)$. Here, we use the local coordinate system depending on $\mathcal{B}$ with the notation

$$
X=\left(X^{\prime}, X_{d}\right) \in \mathbb{R}^{d}, \quad X^{\prime}=\left(X_{1}, \cdots, X_{d-1}\right) \in \mathbb{R}^{d-1}, \quad X_{d} \in \mathbb{R} .
$$

So, $X_{d}=h\left(X^{\prime}\right)$ for all $X^{\prime} \in \prod_{j=1}^{d-1}\left(a_{j}-\delta, b_{j}+\delta\right)$. We shall assume that $\delta>0$ is small enough so that the corresponding concentric balls with radius reduced by $\delta$ still cover $\Gamma$, and that in particular the union of the graphs of $h$ on $\prod_{j=1}^{d-1}\left(a_{j}, b_{j}\right)$ with all open balls $\mathcal{B}$ is still $\Gamma$. 
Moreover, since all $\Gamma$ and $T_{t}(t \in \mathbb{R})$ are smooth, there exists $t_{0}^{\prime} \in(0,1)$ such that, for any $t \in \mathbb{R}$ with $|t| \leq t_{0}^{\prime}$ and for any $X=\left(X^{\prime}, X_{d}\right) \in \Gamma$ with $X^{\prime} \in \prod_{j=1}^{d-1}\left(a_{j}, b_{j}\right)$, the coordinate $\left(T_{t}(X)\right)^{\prime}$ of $T_{t}(X)=\left(\left(T_{t}(X)\right)^{\prime},\left(T_{t}(X)\right)_{d}\right)$ is still in $\prod_{j=1}^{d-1}\left(a_{j}-\delta, b_{j}+\delta\right)$, the domain of $h$. With this setup, we shall prove that there exist $t_{0} \in\left(0, t_{0}^{\prime}\right)$ and $C>0$ such that

$$
\operatorname{dist}\left(T_{t}\left(X_{0}\right), \Gamma\right) \leq C t^{2} \quad \text { if } X_{0}=\left(X_{0}^{\prime}, X_{0 d}\right) \in \Gamma \text { with } X_{0}^{\prime} \in \prod_{j=1}^{d-1}\left(a_{j}, b_{j}\right) \text { and }|t| \leq t_{0} .
$$

This then implies 4.13.

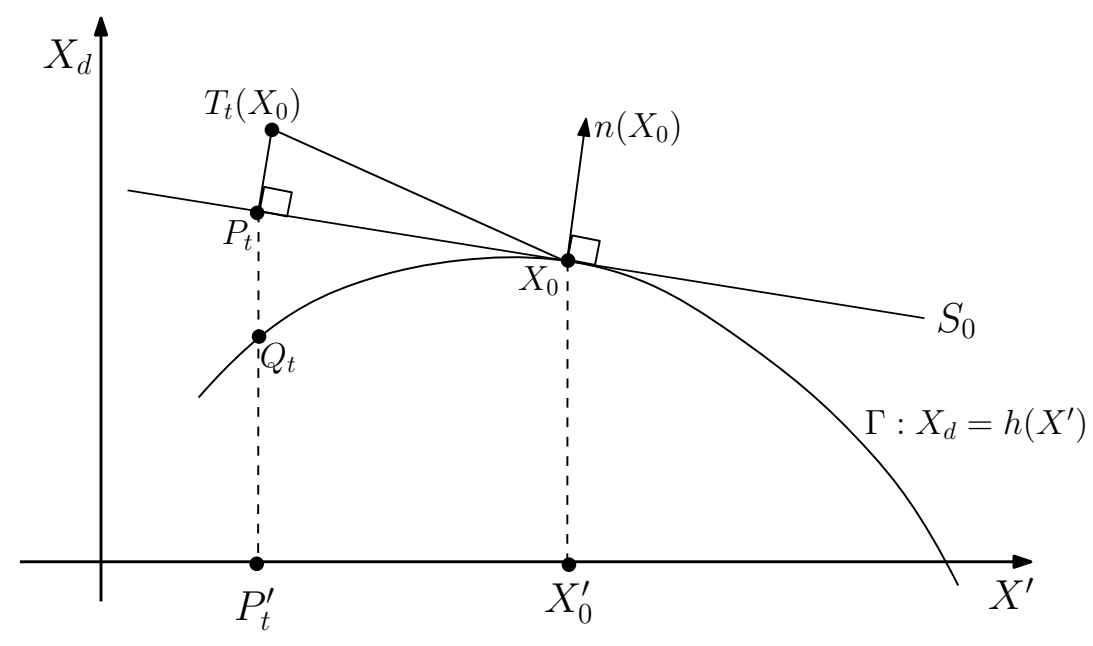

Figure 2: A local graph representation of the surface $\Gamma$.

Let us fix an arbitrary point $X_{0}=\left(X_{0}^{\prime}, X_{0 d}\right) \in \Gamma$ with $X_{0}^{\prime} \in \prod_{j=1}^{d-1}\left(a_{j}, b_{j}\right)$ and $X_{0 d}=h\left(X_{0}^{\prime}\right)$; cf. Figure 2. The equation for the plane, $S_{0}$, that is tangent to the surface $\Gamma$ at $X_{0} \in \Gamma$ is given by

$$
\left(X-X_{0}\right) \cdot n\left(X_{0}\right)=0 \quad \text { with } X=\left(X^{\prime}, X_{d}\right) \in \mathbb{R}^{d},
$$

where $n\left(X_{0}\right)$ is the unit vector normal to $\Gamma$ at $X_{0}$,

$$
n\left(X_{0}\right)=\frac{\left(-\nabla_{X^{\prime}} h\left(X_{0}^{\prime}\right), 1\right)}{\sqrt{1+\left|\nabla_{X^{\prime}} h\left(X_{0}^{\prime}\right)\right|^{2}}}
$$

Let $t \in \mathbb{R}$ with $|t| \leq t_{0}^{\prime}$. Denote by $P_{t}=\left(P_{t}^{\prime}, P_{t d}\right) \in S_{0}$ the point of the orthogonal projection of the vector $T_{t}\left(X_{0}\right)-X_{0}$ on this tangent plane $S_{0}$, given by

$$
P_{t}=-\left[\left(T_{t}\left(X_{0}\right)-X_{0}\right) \cdot n\left(X_{0}\right)\right] n\left(X_{0}\right)+T_{t}\left(X_{0}\right)
$$

Denote

$$
Q_{t}=\left(Q_{t}^{\prime}, Q_{t d}\right)=\left(P_{t}^{\prime}, h\left(P_{t}^{\prime}\right)\right) \in \Gamma .
$$

We show that

$$
\left|T_{t}\left(X_{0}\right)-Q_{t}\right| \leq C t^{2} \quad \text { if }|t| \leq t_{0}
$$


for some constants $t_{0} \in(0,1)$ and $C>0$ independent of $X_{0}$. This should imply (4.15) as

$$
\operatorname{dist}\left(T_{t}\left(X_{0}\right), \Gamma\right) \leq\left|T_{t}\left(X_{0}\right)-Q_{t}\right| .
$$

To prove (4.19), it suffices by the triangle inequality to prove

$$
\begin{aligned}
& \left|T_{t}\left(X_{0}\right)-P_{t}\right| \leq C t^{2}, \\
& \left|P_{t}-Q_{t}\right|=\left|P_{t d}-Q_{t d}\right| \leq C t^{2},
\end{aligned}
$$

for all $t$ with $|t| \leq t_{0}$. Here and below in the proof, $C$ denotes a generic constant independent of $X_{0}$ and $t \in\left[-t_{0}, t_{0}\right]$. Since $V \in C_{\mathrm{c}}^{2}\left(\mathbb{R}^{3}, \mathbb{R}^{3}\right)$, we have by (3.7) that

$$
\begin{aligned}
\partial_{t t} T_{t}(X) & =\partial_{t t} x(t, X)=\partial_{t}(V(x(t, X)))=(\nabla V(x(t, X))) \partial_{t} x(t, X) \\
& =(\nabla V(x(t, X))) V(x(t, X)) \leq C \quad \forall t \in \mathbb{R} \forall X \in \mathbb{R}^{d} .
\end{aligned}
$$

Therefore, by Taylor's expansion, (4.17), (3.7), the fact that $\left|n\left(X_{0}\right)\right|=1$, and the assumption that $V\left(X_{0}\right) \cdot n\left(X_{0}\right)=0$, we have

$$
\begin{aligned}
\left|T_{t}\left(X_{0}\right)-P_{t}\right| & =\left|\left[x\left(t, X_{0}\right)-X_{0}\right] \cdot n\left(X_{0}\right)\right| \\
& \leq\left|x\left(0, X_{0}\right)-X_{0}\right|+\left|t \partial_{t} x\left(0, X_{0}\right) \cdot n\left(X_{0}\right)\right|+C t^{2} \\
& =\left|t V\left(X_{0}\right) \cdot n\left(X_{0}\right)\right|+C t^{2} \\
& =C t^{2},
\end{aligned}
$$

proving 4.20).

Since $V$ is compactly supported, we have by (3.7) and Taylor's expansion that

$$
\left|T_{t}\left(X_{0}\right)-X_{0}\right|=\left|x\left(t, X_{0}\right)-X_{0}\right|=\left|t \partial_{t} x\left(\xi_{t}, X_{0}\right)\right|=|t|\left|V\left(x\left(\xi_{t}, X_{0}\right)\right)\right| \leq C|t|,
$$

where $\xi_{t}$ is in between 0 and $t$. This and 4.20 imply

$$
\left|P_{t}^{\prime}-X_{0}^{\prime}\right| \leq\left|P_{t}-X_{0}\right| \leq\left|P_{t}-T_{t}\left(X_{0}\right)\right|+\left|T_{t}\left(X_{0}\right)-X_{0}\right| \leq C|t| \quad \text { if }|t|<t_{0} .
$$

By (4.18) and Taylor's expansion,

$$
\begin{aligned}
Q_{t d} & =h\left(P_{t}^{\prime}\right) \\
& =h\left(X_{0}^{\prime}\right)+\nabla_{X^{\prime}} h\left(X_{0}^{\prime}\right) \cdot\left(P_{t}^{\prime}-X_{0}^{\prime}\right)+\frac{1}{2} \nabla_{X^{\prime}}^{2} h\left(Y_{t}^{\prime}\right)\left(P_{t}^{\prime}-X_{0}^{\prime}\right) \cdot\left(P_{t}^{\prime}-X_{0}^{\prime}\right)
\end{aligned}
$$

for some $Y_{t}^{\prime} \in \prod_{j=1}^{d-1}\left(a_{j}, b_{j}\right)$. Since $\left(P_{t}-X_{0}\right) \cdot n\left(X_{0}\right)=0, X_{0 d}=h\left(X_{0}^{\prime}\right)$, and $n\left(X_{0}\right)$ is given by (4.16), we have that

$$
P_{t d}=h\left(X_{0}^{\prime}\right)+\nabla_{X^{\prime}} h\left(X_{0}\right) \cdot\left(P_{t}^{\prime}-X_{0}^{\prime}\right) .
$$

This, together with (4.23) and 4.22), implies (4.21). The constant $C$ depends on $h$ and $V$, and hence on $\Gamma$ and $V$ only. 
We now prove (4.14). We only consider the case $s=-$, as the case $s=+$ can be treated similarly. Moreover, for $t=0$, the inequality in (4.14) holds true obviously, since $T_{0}$ is the identity map. So, let $t \in \mathbb{R}$ be such that $0<|t| \leq t_{0}$. We further assume that $0<t \leq t_{0}$ as the case $-t_{0} \leq t<0$ is similar.

We claim that

$$
\Omega_{-} \Delta T_{t}\left(\Omega_{-}\right):=\left(\Omega_{-} \backslash T_{t}\left(\Omega_{-}\right)\right) \cup\left(T_{t}\left(\Omega_{-}\right) \backslash \Omega_{-}\right) \subseteq\left\{X \in \mathbb{R}^{d}: \operatorname{dist}(X, \Gamma) \leq C t^{2}\right\},
$$

where the constant $C$ is exactly the same as in 4.13$)$. In fact, if $X \in \Omega_{-} \backslash T_{t}\left(\Omega_{-}\right)$, then $X \in \Omega_{-}$and $T_{-t}(X) \in \overline{\Omega_{+}}$. Hence, $\left\{T_{s}\left(T_{-t}(X)\right)\right\}_{0 \leq s \leq t}$ is a $C^{3}$-curve in $\mathbb{R}^{d}$, connecting one endpont $T_{0}\left(T_{-t}(X)\right)=T_{-t}(X) \in \overline{\Omega_{+}}$to the other $T_{t}\left(T_{-t}(X)\right)=X \in \Omega_{-}$. Since $\Gamma$ is a closed hypersurface in $\mathbb{R}^{d}$, there must exist $s_{0} \in(0, t)$ such that $T_{s_{0}}\left(T_{-t}(X)\right)=T_{s_{0}-t}(X) \in \Gamma$. Hence, by 4.13 with $t-s_{0}$ and $T_{s_{0}-t}(X)$ replacing $t$ and $X$, respectively,

$$
\operatorname{dist}(X, \Gamma)=\operatorname{dist}\left(T_{t-s_{0}}\left(T_{s_{0}-t}(X)\right), \Gamma\right) \leq C\left(t-s_{0}\right)^{2} \leq C t^{2} .
$$

Similarly, if $X \in T_{t}\left(\Omega_{-}\right) \backslash \Omega_{-}$, then $\operatorname{dist}(X, \Gamma) \leq C t^{2}$. Hence, 4.24 holds true.

By 4.24, we have

$$
\left|\left\{X \in \mathbb{R}^{d}: \chi_{\Omega_{-}(t)} \neq \chi_{\Omega_{-}(X)}\right\}\right|=\left|\Omega_{-} \Delta T_{t}\left(\Omega_{-}\right)\right| \leq\left|\left\{X \in \mathbb{R}^{d}: \operatorname{dist}(X, \Gamma) \leq C t^{2}\right\}\right| .
$$

This implies (4.14) (for $\mathrm{s}=-$ ), as the right-hand side of the above inequality is bounded by $C t^{2}$ if $|t| \leq t_{0}$ with a possible smaller $t_{0}$ (cf. Lemma 2.1 in $[33]$ ).

\subsection{Continuity and Differentiability}

Let $\Gamma$ be a dielectric boundary satisfying the assumptions in A1 of Subsection 2.1 and $V \in \mathcal{V}$ (cf. (3.6)). Let $\left\{T_{t}\right\}_{t \in \mathbb{R}}$ be the corresponding family of diffeomorphisms defined by (3.7). Let $\hat{\phi} \in W^{1,1}(\Omega)$ satisfy 2.3 . We consider the approximations $\hat{\phi} \circ T_{t}$. Note that $\hat{\phi} \circ T_{t}-\hat{\phi}$ and $\nabla \hat{\phi} \cdot V$ vanish in any small neighborhood of $\cup_{i=1}^{N} x_{i}$, as $V(X)=0$ and $T_{t}(X)=X$ for any $X$ in such a neighborhood and any $t \in \mathbb{R}$.

Lemma 4.2. Let $\hat{\phi} \in \hat{\phi}_{\mathrm{C}}+H^{1}(\Omega)$ satisfy 2.3. We have

$$
\lim _{t \rightarrow 0}\left\|\hat{\phi} \circ T_{t}-\hat{\phi}\right\|_{H^{1}(\Omega)}=0 .
$$

Moreover, $\nabla \hat{\phi} \cdot V \in H^{1}(\Omega)$ and

$$
\lim _{t \rightarrow 0}\left\|\frac{\hat{\phi} \circ T_{t}-\hat{\phi}}{t}-\nabla \hat{\phi} \cdot V\right\|_{H^{1}(\Omega)}=0 .
$$

Proof. Let $\sigma>0$ be such that $B_{\sigma}:=\cup_{i=1}^{N} B\left(x_{i}, \sigma\right) \subset \Omega$ and $V=0$ on $B_{\sigma}$. Then, there exists $\tilde{\phi} \in C^{\infty}(\Omega) \cap H^{2}(\Omega)$ such that $\tilde{\phi}=0$ in $B_{\sigma / 2}$ and $\tilde{\phi}=\hat{\phi}$ a.e. in $\Omega \backslash B_{\sigma}$. These imply that 
$\tilde{\phi} \circ T_{t}-\tilde{\phi}=\hat{\phi} \circ T_{t}-\hat{\phi}$, and $\nabla \tilde{\phi} \cdot V=\nabla \hat{\phi} \cdot V$ a.e. in $\Omega$ for all $t$. This implies that $\nabla \hat{\phi} \cdot V \in H^{1}(\Omega)$. Moreover, it follows from (4.11) that

$$
\lim _{t \rightarrow 0}\left\|\hat{\phi} \circ T_{t}-\hat{\phi}\right\|_{H^{1}(\Omega)}=\lim _{t \rightarrow 0}\left\|\tilde{\phi} \circ T_{t}-\tilde{\phi}\right\|_{H^{1}(\Omega)}=0
$$

implying 4.25), and from 4.12 that

$$
\lim _{t \rightarrow 0}\left\|\frac{\hat{\phi} \circ T_{t}-\hat{\phi}}{t}-\nabla \hat{\phi} \cdot V\right\|_{H^{1}(\Omega)}=\lim _{t \rightarrow 0}\left\|\frac{\tilde{\phi} \circ T_{t}-\tilde{\phi}}{t}-\nabla \tilde{\phi} \cdot V\right\|_{H^{1}(\Omega)}=0,
$$

implying 4.26).

We recall that $\phi_{\Gamma, \infty} \in H^{1}(\Omega) \cap C(\bar{\Omega})$ is the unique weak solution to the boundary-value problem (1.7), defined in 2.5 . Similarly, $\phi_{\Gamma_{t}, \infty} \in H^{1}(\Omega) \cap C(\bar{\Omega})$ for each $t \in \mathbb{R}$ is the unique weak solution to the same boundary-value problem with $\Gamma_{t}=T_{t}(\Gamma)$ replacing $\Gamma$.

Lemma 4.3. (1) There exists a unique $\zeta_{\Gamma, V} \in H_{0}^{1}(\Omega)$ such that

$$
\int_{\Omega} \varepsilon_{\Gamma} \nabla \zeta_{\Gamma, V} \cdot \nabla \eta d x=-\int_{\Omega} \varepsilon_{\Gamma} A_{V}^{\prime}(0) \nabla \phi_{\Gamma, \infty} \cdot \nabla \eta d x \quad \forall \eta \in H_{0}^{1}(\Omega),
$$

where $A_{V}^{\prime}(0)$ is defined in (4.6). Moreover, the mapping $V \mapsto \zeta_{\Gamma, V}$ is linear in $V$, i.e.,

$$
\zeta_{\Gamma, c_{1} V_{1}+c_{2} V_{2}}=c_{1} \zeta_{\Gamma, V_{1}}+c_{2} \zeta_{\Gamma, V_{2}} \quad \text { for all } V_{1}, V_{2} \in \mathcal{V} \text { and } c_{1}, c_{2} \in \mathbb{R} .
$$

(2) By modifying the value of $\zeta_{\Gamma, V}$ on a set of zero Lebesgue measure, we have that $\zeta_{\Gamma, V}^{\mathrm{s}} \in$ $H^{2}\left(\Omega_{\mathrm{s}}\right) \cap C^{1}\left(\Omega_{\mathrm{s}}\right)$ for $\mathrm{s}=-$ or + . Moreover,

$$
\begin{aligned}
& \Delta \zeta_{\Gamma, V}=-\nabla \cdot\left[A_{V}^{\prime}(0) \nabla \phi_{\Gamma, \infty}\right]=\Delta\left(\nabla \phi_{\Gamma, \infty} \cdot V\right) \quad \text { in } \Omega_{-} \cup \Omega_{+}, \\
& \llbracket \varepsilon_{\Gamma} \partial_{n} \zeta_{\Gamma, V} \rrbracket_{\Gamma}=-\llbracket \varepsilon_{\Gamma} A_{V}^{\prime}(0) \nabla \phi_{\Gamma, \infty} \cdot n \rrbracket_{\Gamma} \quad \text { on } \Gamma .
\end{aligned}
$$

(3) We have

$$
\begin{aligned}
& \lim _{t \rightarrow 0}\left\|\phi_{\Gamma_{t}, \infty} \circ T_{t}-\phi_{\Gamma, \infty}\right\|_{H^{1}(\Omega)}=0, \\
& \lim _{t \rightarrow 0}\left\|\frac{\phi_{\Gamma_{t}, \infty} \circ T_{t}-\phi_{\Gamma, \infty}}{t}-\zeta_{\Gamma, V}\right\|_{H^{1}(\Omega)}=0 .
\end{aligned}
$$

(4) If $V \cdot n=0$ on $\Gamma$, then $\zeta_{\Gamma, V}=\nabla \phi_{\Gamma, \infty} \cdot V$ in $\Omega$.

Proof. (1) The existence and uniqueness of $\zeta_{\Gamma, V} \in H_{0}^{1}(\Omega)$ that satisfies (4.27) follow from the Lax-Milgram Lemma 22, 25]. By (4.6), $A_{V}^{\prime}(0)$ is linear in $V$. Therefore, by the definition (4.27) of $\zeta_{\Gamma, V} \in H_{0}^{1}(\Omega), \zeta_{\Gamma, V}$ is linear in $V$.

(2) Let $\mathrm{s}$ denote - or + . Note by (2.6), (3.6), and (4.6) that $A_{V}^{\prime}(0) \nabla \phi_{\Gamma, \infty} \in C^{1}\left(\Omega_{\mathrm{s}}\right) \cap$ $H^{1}\left(\Omega_{\mathrm{s}}\right)$. For any $\eta \in C_{\mathrm{c}}^{1}(\Omega)$ with $\operatorname{supp}(\eta) \subset \Omega_{\mathrm{s}}$, we have by 4.27 and the Divergence Theorem that

$$
\int_{\Omega_{\mathrm{s}}} \varepsilon_{\mathrm{s}} \nabla \zeta_{\Gamma, V} \cdot \nabla \eta d x=\int_{\Omega_{\mathrm{s}}} \varepsilon_{\mathrm{s}} \nabla \cdot\left[A_{V}^{\prime}(0) \nabla \phi_{\Gamma, \infty}\right] \eta d x
$$


Hence, $-\Delta \zeta_{\Gamma, V}=\nabla \cdot\left[A_{V}^{\prime}(0) \nabla \phi_{\Gamma, \infty}\right]$ in $\Omega_{\mathrm{s}}$. Since the right-hand side is in $L^{2}\left(\Omega_{\mathrm{s}}\right) \cap C\left(\Omega_{\mathrm{s}}\right)$, it follows from the elliptic regularity theory 25,32 that $\zeta_{\Gamma, V}^{\mathrm{s}} \in H^{2}\left(\Omega_{\mathrm{s}}\right) \cap C^{1}\left(\Omega_{\mathrm{s}}\right)$, after a possible modification of the value of $\zeta_{\Gamma, V}$ on a set of zero Lebesgue measure. Moreover, the first equality in 4.28 follows.

Let us denote by $V^{i}(i=1,2,3)$ the components of $V$. With the conventional summation notation (i.e., repeated indices are summed), we have by (4.6), (2.6), and (2.8) that

$$
\begin{aligned}
- & \nabla \cdot\left(A_{V}^{\prime}(0) \nabla \phi_{\Gamma, \infty}\right) \\
& =\nabla \cdot\left[\nabla V+(\nabla V)^{T}-(\nabla \cdot V) I\right] \nabla \phi_{\Gamma, \infty} \\
& =\partial_{i}\left(\partial_{j} V^{i} \partial_{j} \phi_{\Gamma, \infty}+\partial_{i} V^{j} \partial_{j} \phi_{\Gamma, \infty}-\partial_{k} V^{k} \partial_{i} \phi_{\Gamma, \infty}\right) \\
& =2 \partial_{i j} \phi_{\Gamma, \infty} \partial_{i} V^{j}+\partial_{i i} V^{j} \partial_{j} \phi_{\Gamma, \infty} \\
& \left.=\partial_{i i} \partial_{j} \phi_{\Gamma, \infty} V^{j}+2 \partial_{i j} \phi_{\Gamma, \infty} \partial_{i} V^{j}+\partial_{i i} V^{j} \partial_{j} \phi_{\Gamma, \infty} \quad \text { [since } \partial_{i i} \phi_{\Gamma, \infty}=0\right] \\
& =\partial_{i i}\left(\partial_{j} \phi_{\Gamma, \infty} V^{j}\right) \\
& =\Delta\left(\nabla \phi_{\Gamma, \infty} \cdot V\right) \quad \text { in } \Omega_{-} \cup \Omega_{+},
\end{aligned}
$$

implying the second equation in (4.28).

Since $\zeta_{\Gamma, V} \in H_{0}^{1}(\Omega)$ and $\Delta \zeta_{\Gamma, V} \in L^{2}\left(\Omega_{\mathrm{s}}\right)$ for s being - or + , and since the unit normal $n$ at the $\Gamma$ points from $\Omega_{-}$to $\Omega_{+}$, we have by the Divergence Theorem that both sides of the equation in 4.27) are

$$
\begin{aligned}
\int_{\Omega} \varepsilon_{\Gamma} \nabla \zeta_{\Gamma, V} \cdot \nabla \eta d x & =\int_{\Omega_{-}} \varepsilon_{-} \nabla \zeta_{\Gamma, V} \cdot \nabla \eta d x+\int_{\Omega_{+}} \varepsilon_{+} \nabla \zeta_{\Gamma, V} \cdot \nabla \eta d x \\
& =-\int_{\Omega_{-}} \varepsilon_{-} \Delta \zeta_{\Gamma, V} \eta d x-\int_{\Omega_{+}} \varepsilon_{+} \Delta \zeta_{\Gamma, V} \eta d x-\int_{\Gamma} \llbracket \varepsilon_{\Gamma} \partial_{n} \zeta_{\Gamma, V} \rrbracket_{\Gamma} \eta d S,
\end{aligned}
$$

and

$$
\begin{aligned}
- & \int_{\Omega} \varepsilon_{\Gamma} A_{V}^{\prime}(0) \nabla \phi_{\Gamma, \infty} \cdot \nabla \eta d x \\
= & -\int_{\Omega_{-}} \varepsilon_{-} A_{V}^{\prime}(0) \nabla \phi_{\Gamma, \infty} \cdot \nabla \eta d x-\int_{\Omega_{+}} \varepsilon_{+} A_{V}^{\prime}(0) \nabla \phi_{\Gamma, \infty} \cdot \nabla \eta d x \\
= & \int_{\Omega_{-}} \varepsilon_{-} \nabla \cdot\left(A_{V}^{\prime}(0) \nabla \phi_{\Gamma, \infty}\right) \eta d x+\int_{\Omega_{+}} \varepsilon_{+} \nabla \cdot\left(A_{V}^{\prime}(0) \nabla \phi_{\Gamma, \infty}\right) \eta d x \\
& +\int_{\Gamma} \llbracket \varepsilon_{\Gamma} A_{V}^{\prime}(0) \nabla \phi_{\Gamma, \infty} \cdot n \rrbracket_{\Gamma} \eta d S,
\end{aligned}
$$

respectively. These, together with and (4.28), imply (4.29).

(3) Replacing $\Gamma, \phi_{\Gamma, \infty}$, and $\eta$ by $\Gamma_{t}, \phi_{\Gamma_{t}, \infty}$, and $\eta \circ T_{t}^{-1}$ for $t \in \mathbb{R}$, respectively, in the weak formulation (2.5), we get by the change of variable $x=T_{t}(X)$ that

$$
\int_{\Omega} \varepsilon_{\Gamma} A_{V}(t) \nabla\left(\phi_{\Gamma_{t}, \infty} \circ T_{t}\right) \cdot \nabla \eta d X=0 \quad \forall \eta \in H_{0}^{1}(\Omega) .
$$


This and 2.5 imply for any $\eta \in H_{0}^{1}(\Omega)$ that

$$
\int_{\Omega} \varepsilon_{\Gamma} \nabla\left(\phi_{\Gamma_{t}, \infty} \circ T_{t}-\phi_{\Gamma, \infty}\right) \cdot \nabla \eta d X=\int_{\Omega} \varepsilon_{\Gamma}\left[I-A_{V}(t)\right] \nabla\left(\phi_{\Gamma_{t}, \infty} \circ T_{t}\right) \cdot \nabla \eta d X .
$$

It follows from a change of variable, (4.2), 4.3$),(4.7)$, and $(4.8)$ that $\left\|\nabla\left(\phi_{\Gamma_{t}, \infty} \circ T_{t}\right)\right\|_{L^{2}(\Omega)}$ is bounded uniformly in $t$. Setting $\eta=\phi_{\Gamma_{t}, \infty} \circ T_{t}-\phi_{\Gamma, \infty} \in H_{0}^{\mathrm{T}}(\Omega)$ in (4.33), we then obtain (4.30) by (4.7), (4.8), and the Cauchy-Schwarz and Poincaré inequalities.

Dividing both sides of (4.33) by $t \neq 0$ and setting now $\eta=\left(\phi_{\Gamma_{t}, \infty} \circ T_{t}-\phi_{\Gamma, \infty}\right) / t-\zeta_{\Gamma, V}$ in the resulting equation and also in (4.27), we have by the Cauchy-Schwarz inequality that

$$
\begin{aligned}
\int_{\Omega} \varepsilon_{\Gamma} & \left|\nabla\left(\frac{\phi_{\Gamma_{t}, \infty} \circ T_{t}-\phi_{\Gamma, \infty}}{t}-\zeta_{\Gamma, V}\right)\right|^{2} d X \\
= & \int_{\Omega} \varepsilon_{\Gamma}\left[\frac{I-A_{V}(t)}{t}+A_{V}^{\prime}(0)\right] \nabla\left(\phi_{\Gamma_{t}, \infty} \circ T_{t}\right) \cdot \nabla\left(\frac{\phi_{\Gamma_{t}, \infty} \circ T_{t}-\phi_{\Gamma, \infty}}{t}-\zeta_{\Gamma, V}\right) d X \\
& \left.+\int_{\Omega} \varepsilon_{\Gamma} A_{V}^{\prime}(0) \nabla\left[\phi_{\Gamma, \infty}-\phi_{\Gamma_{t}, \infty} \circ T_{t}\right)\right] \cdot \nabla\left(\frac{\phi_{\Gamma_{t}, \infty} \circ T_{t}-\phi_{\Gamma, \infty}}{t}-\zeta_{\Gamma, V}\right) d X \\
\leq & C\left\|\frac{A_{V}(t)-I-t A_{V}^{\prime}(0)}{t}\right\|_{L^{\infty}(\Omega)}\left\|\phi_{\Gamma_{t}, \infty} \circ T_{t}\right\|_{H^{1}(\Omega)}\left\|\frac{\phi_{\Gamma_{t}, \infty} \circ T_{t}-\phi_{\Gamma, \infty}}{t}-\zeta_{\Gamma, V}\right\|_{H^{1}(\Omega)} \\
& +C\left\|\phi_{\Gamma, \infty}-\phi_{\Gamma_{t}, \infty} \circ T_{t}\right\|_{H^{1}(\Omega)}\left\|\frac{\phi_{\Gamma_{t}, \infty} \circ T_{t}-\phi_{\Gamma, \infty}}{t}-\zeta_{\Gamma, V}\right\|_{H^{1}(\Omega)} .
\end{aligned}
$$

This, together with Poincaré's inequality, 4.7), 4.8), and (4.30), leads to (4.31).

(4) Assume now $V \cdot n=0$ on $\Gamma$. Recall from subsection 3.2 that the signed distance to $\Gamma$, $\phi: \mathbb{R}^{3} \rightarrow \mathbb{R}$, which is negative in $\Omega_{-}$and positive outside $\Gamma$, is in fact a $C^{3}$-function and also $\nabla \phi \neq 0$ in $\mathcal{N}_{0}(\Gamma)$, a neighborhood of $\Gamma$ in $\Omega$; cf. (3.5). Moreover, $n=\nabla \phi$ on $\Gamma$. We define $n=\nabla \phi$ on $\mathcal{N}_{0}(\Gamma)$. Now, since $V \cdot n=0$ on $\Gamma$, by Lemma 4.1 , there exists $t_{0}=t_{0}(V)>0$ and a constant $C_{0}>0$ which may depend on $\Gamma$, such that dist $(x, \Gamma) \leq C_{0} t^{2}$ for all $x \in \Gamma_{t}$ and all $t \in\left[-t_{0}, t_{0}\right]$. Let $D(t)=\left\{x \in \Omega: \operatorname{dist}(x, \Gamma) \leq C_{0} t^{2}\right\}$. Then $\Gamma_{t} \subset D(t)$, and hence $\varepsilon_{\Gamma_{t}}=\varepsilon_{\Gamma}$ on $\Omega \backslash D(t)$, for all $|t| \leq t_{0}$. Moreover, the measure $|D(t)|=O\left(t^{2}\right)$ as $t \rightarrow 0$; cf. Lemma 2.1 in 33 .

Let $h_{t}=\left(\phi_{\Gamma_{t}, \infty}-\phi_{\Gamma, \infty}\right) / t$ with $|t| \leq t_{0}$. We have now by $(1.7)$ and that with $\Gamma_{t}$ replacing $\Gamma$ that

$$
\int_{\Omega} \varepsilon_{\Gamma_{t}} \nabla h_{t} \cdot \nabla \eta d x=-\int_{\Omega} \frac{\varepsilon_{\Gamma_{t}}-\varepsilon_{\Gamma}}{t} \nabla \phi_{\Gamma, \infty} \cdot \nabla \eta d x \quad \forall \eta \in H_{0}^{1}(\Omega) .
$$

Setting $\eta=h_{t} \in H_{0}^{1}(\Omega)$, we have by the uniform boundedness of $\nabla \phi_{\Gamma_{t}, \infty}$ (cf. (2.7)), the Poincaré and Cauchy-Schwarz inequalities, and the fact that $|D(t)|=O\left(t^{2}\right)$ as $t \rightarrow 0$ that

$$
\left\|h_{t}\right\|_{H^{1}(\Omega)} \leq C\left(\int_{D(t)}\left|\frac{\varepsilon_{\Gamma_{t}}-\varepsilon_{\Gamma}}{t}\right|^{2} d x\right)^{1 / 2} \leq C,
$$

where $C>0$ is a generic constant, independent of $t$. Thus, there exists a subsequence of $h_{t}$ $\left(|t| \leq t_{0}\right)$, not relabeled, and some $h \in H_{0}^{1}(\Omega)$, such that $h_{t} \rightarrow h$ weakly in $H^{1}(\Omega)$ as $t \rightarrow 0$. 
Working on this subsequence, we have by (2.5) (with $\phi_{\Gamma_{t}, \infty}$ replacing $\left.\phi_{\Gamma, \infty}\right),(4.34),(2.7)$, and the fact that the measure $|D(t)|=O\left(t^{2}\right) \rightarrow 0$ as $t \rightarrow 0$ that for any $\eta \in H_{0}^{1}(\Omega)$

$$
\begin{aligned}
\left|\int_{\Omega} \varepsilon_{\Gamma} \nabla h_{t} \cdot \nabla \eta d x\right| & =\left|\int_{\Omega}\left(\varepsilon_{\Gamma}-\varepsilon_{\Gamma_{t}}\right) \nabla h_{t} \cdot \nabla \eta d x-\int_{\Omega} \frac{\varepsilon_{\Gamma_{t}}-\varepsilon_{\Gamma}}{t} \nabla \phi_{\Gamma, \infty} \cdot \nabla \eta d x\right| \\
& \leq\left|\int_{D(t)}\left(\varepsilon_{\Gamma_{t}}-\varepsilon_{\Gamma}\right) \nabla h_{t} \cdot \nabla \eta d x\right|+\left|\int_{D(t)} \frac{\varepsilon_{\Gamma_{t}}-\varepsilon_{\Gamma}}{t} \nabla \phi_{\Gamma, \infty} \cdot \nabla \eta d x\right| \\
& \leq C\left[\left\|h_{t}\right\|_{H^{1}(\Omega)}+\frac{1}{|t|}|D(t)|^{1 / 2}\right]\left(\int_{D(t)}|\nabla \eta|^{2} d x\right)^{1 / 2} \\
& \leq C\left(\int_{D(t)}|\nabla \eta|^{2} d x\right)^{1 / 2} \\
& \rightarrow 0 \quad \text { as } t \rightarrow 0 .
\end{aligned}
$$

Since $h_{t} \rightarrow h$ weakly in $H^{1}(\Omega)$, we have

$$
\int_{\Omega} \varepsilon_{\Gamma} \nabla h \cdot \nabla \eta d x=0 \quad \forall \eta \in H_{0}^{1}(\Omega) .
$$

Setting $\eta=h \in H_{0}^{1}(\Omega)$, we see that $h=0$ in $H_{0}^{1}(\Omega)$.

We now show that $\zeta_{\Gamma}=\nabla \phi_{\Gamma, \infty} \cdot V$ in $\Omega$. Let $\eta \in L^{2}(\Omega)$ and $t \neq 0$. Since $h_{t} \rightarrow h=0$ weakly in $H^{1}(\Omega)$, we have by the properties of the transformations $T_{t}(t \in \mathbb{R})(4.9),(4.12)$, and (4.1) -4.3 that

$$
\begin{aligned}
\int_{\Omega} & \frac{\phi_{\Gamma t, \infty} \circ T_{t}-\phi_{\Gamma, \infty}}{t} \eta d X \\
= & \int_{\Omega} \frac{\phi_{\Gamma t, \infty} \circ T_{t}-\phi_{\Gamma, \infty} \circ T_{t}}{t} \eta d X+\int_{\Omega} \frac{\phi_{\Gamma, \infty} \circ T_{t}}{t} \eta d X-\int_{\Omega} \frac{\phi_{\Gamma, \infty} \eta}{t} d X \\
= & \int_{\Omega} h_{t}\left(\eta \circ T_{t}^{-1}\right) \operatorname{det} \nabla T_{t}^{-1} d x+\int_{\Omega} \frac{\phi_{\Gamma, \infty}}{t}\left(\eta \circ T_{t}^{-1}\right) \operatorname{det} \nabla T_{t}^{-1} d x-\int_{\Omega} \frac{\phi_{\Gamma, \infty} \eta}{t} d x \\
= & \int_{\Omega} h_{t}\left(\eta \circ T_{t}^{-1}\right) \operatorname{det} \nabla T_{t}^{-1} d x \\
& +\int_{\Omega} \phi_{\Gamma, \infty}\left(\frac{\eta \circ T_{t}^{-1}-\eta}{t} \operatorname{det} \nabla T_{t}^{-1}+\eta \frac{\operatorname{det} \nabla T_{t}^{-1}-1}{t}\right) d x \\
\rightarrow & -\int_{\Omega} \phi_{\Gamma, \infty} \nabla \eta \cdot V d x-\int_{\Omega} \phi_{\Gamma, \infty} \eta(\nabla \cdot V) d x \\
= & \int_{\Omega}\left(\nabla \phi_{\Gamma, \infty} \cdot V\right) \eta d x \quad \text { as } t \rightarrow 0 .
\end{aligned}
$$

This and 4.31), together with the arbitrariness of $\eta \in L^{2}(\Omega)$, imply that $\zeta_{\Gamma, V}=\nabla \phi_{\Gamma, \infty} \cdot V$ in $\Omega$. 
We recall that $\hat{\phi}_{\Gamma, \infty}$ is determined by 2.11 and the boundary condition $\hat{\phi}_{\Gamma, \infty}=\phi_{\infty}$ on $\partial \Omega$. For each $t \in \mathbb{R}$, we denote by $\hat{\phi}_{\Gamma_{t}, \infty}$ the unique function that is defined by 2.11 ) with $\Gamma_{t}$ replacing $\Gamma$ and the same boundary condition $\hat{\phi}_{\Gamma_{t}, \infty}=\phi_{\infty}$ on $\partial \Omega$. Note that all the singularities $x_{i}(i=1, \ldots, N)$ are outside the support of the vector field $V$.

Lemma 4.4. (1) There exists a unique $\xi_{\Gamma, V} \in H_{0}^{1}(\Omega)$ such that

$$
\int_{\Omega} \varepsilon_{\Gamma} \nabla \xi_{\Gamma, V} \cdot \nabla \eta d x=-\int_{\Omega} \varepsilon_{\Gamma} A_{V}^{\prime}(0) \nabla \hat{\phi}_{\Gamma, \infty} \cdot \nabla \eta d x \quad \forall \eta \in H_{0}^{1}(\Omega) .
$$

(2) By modifying the value of $\xi_{\Gamma, V}$ on a set of zero Lebesgue measure, we have that $\xi_{\Gamma, V}^{\mathrm{s}} \in$ $H^{2}\left(\Omega_{\mathrm{s}}\right) \cap C^{1}\left(\Omega_{\mathrm{s}}\right)$ for $\mathrm{s}=-$ or + . Moreover,

$$
\begin{aligned}
& \Delta \xi_{\Gamma, V}=-\nabla \cdot A_{V}^{\prime}(0) \nabla \hat{\phi}_{\Gamma, \infty}=\Delta\left(\nabla \hat{\phi}_{\Gamma, \infty} \cdot V\right) \quad \text { in } \Omega_{-} \cup \Omega_{+}, \\
& \llbracket \varepsilon_{\Gamma} \partial_{n} \xi_{\Gamma, V} \rrbracket_{\Gamma}=-\llbracket \varepsilon_{\Gamma} A_{V}^{\prime}(0) \partial_{n} \hat{\phi}_{\Gamma, \infty} \rrbracket_{\Gamma} \quad \text { on } \Gamma .
\end{aligned}
$$

(3) We have

$$
\begin{aligned}
& \lim _{t \rightarrow 0}\left\|\hat{\phi}_{\Gamma_{t}, \infty} \circ T_{t}-\hat{\phi}_{\Gamma, \infty}\right\|_{H^{1}(\Omega)}=0, \\
& \lim _{t \rightarrow 0}\left\|\frac{\hat{\phi}_{\Gamma_{t}, \infty} \circ T_{t}-\hat{\phi}_{\Gamma, \infty}}{t}-\xi_{\Gamma, V}\right\|_{H^{1}(\Omega)}=0 .
\end{aligned}
$$

Proof. The proof is the same as and simpler than that of the next lemma, Lemma 4.5, as there is an extra term $B$ there, which can be set to 0 here. The only exception is the second equality in 4.36) which can be obtained by the calculations same as in 4.32.

We recall that $\psi_{\Gamma} \in \hat{\phi}_{\mathrm{C}}+H^{1}(\Omega) \cap C(\bar{\Omega})=\hat{\phi}_{\Gamma, \infty}+H^{1}(\Omega) \cap C(\bar{\Omega})$ is the unique weak solution to the boundary-value problem of the dielectric-boundary PB equation (1.8); cf. Definition 2.1. For each $t \in \mathbb{R}$, we denote by $\psi_{\Gamma_{t}} \in \hat{\phi}_{\mathrm{C}}+H^{1}(\Omega) \cap C(\bar{\Omega})$ the unique solution to the same boundary-value problem with $\Gamma_{t}$ replacing $\Gamma$.

Lemma 4.5. (1) There exists a unique $\omega_{\Gamma, V} \in H_{0}^{1}(\Omega)$ such that

$$
\begin{aligned}
\int_{\Omega} & {\left[\varepsilon_{\Gamma} \nabla \omega_{\Gamma, V} \cdot \nabla \eta+\chi_{+} B^{\prime \prime}\left(\psi_{\Gamma}-\frac{\phi_{\Gamma, \infty}}{2}\right) \omega_{\Gamma, V} \eta\right] d x } \\
= & -\int_{\Omega} \varepsilon_{\Gamma} A_{V}^{\prime}(0) \nabla \psi_{\Gamma} \cdot \nabla \eta d x \\
& -\int_{\Omega_{+}}\left[(\nabla \cdot V) B^{\prime}\left(\psi_{\Gamma}-\frac{\phi_{\Gamma, \infty}}{2}\right)-\frac{\zeta_{\Gamma, V}}{2} B^{\prime \prime}\left(\psi_{\Gamma}-\frac{\phi_{\Gamma, \infty}}{2}\right)\right] \eta d x \quad \forall \eta \in H_{0}^{1}(\Omega) .
\end{aligned}
$$

(2) By modifying the value of $\omega_{\Gamma, V}$ on a set of zero Lebesgue measure, we have that $\omega_{\Gamma, V}^{\mathrm{s}} \in$ $H^{2}\left(\Omega_{\mathrm{s}}\right) \cap C^{1}\left(\Omega_{\mathrm{s}}\right)$ for $\mathrm{s}=-$ or + . Moreover,

$$
\Delta \omega_{\Gamma, V}=-\nabla \cdot A_{V}^{\prime}(0) \nabla \psi_{\Gamma}=\Delta\left(\nabla \psi_{\Gamma} \cdot V\right) \quad \text { in } \Omega_{-},
$$




$$
\begin{aligned}
& \varepsilon_{+} \Delta \omega_{\Gamma, V}-B^{\prime \prime}\left(\psi_{\Gamma}-\frac{\phi_{\Gamma, \infty}}{2}\right) \omega_{\Gamma, V}=-\varepsilon_{+} \nabla \cdot A_{V}^{\prime}(0) \nabla \psi_{\Gamma} \\
& +(\nabla \cdot V) B^{\prime}\left(\psi_{\Gamma}-\frac{\phi_{\Gamma, \infty}}{2}\right)-\frac{\zeta_{\Gamma, V}}{2} B^{\prime \prime}\left(\psi_{\Gamma}-\frac{\phi_{\Gamma, \infty}}{2}\right) \quad \text { in } \Omega_{+}, \\
& \llbracket \varepsilon_{\Gamma} \partial_{n} \omega_{\Gamma, V} \rrbracket_{\Gamma}=-\llbracket \varepsilon_{\Gamma} A_{V}^{\prime}(0) \partial_{n} \psi_{\Gamma} \rrbracket_{\Gamma} \quad \text { on } \Gamma .
\end{aligned}
$$

(3) We have

$$
\begin{aligned}
& \lim _{t \rightarrow 0}\left\|\psi_{\Gamma_{t}} \circ T_{t}-\psi_{\Gamma}\right\|_{H^{1}(\Omega)}=0, \\
& \lim _{t \rightarrow 0}\left\|\frac{\psi_{\Gamma_{t}} \circ T_{t}-\psi_{\Gamma}}{t}-\omega_{\Gamma, V}\right\|_{H^{1}(\Omega)}=0 .
\end{aligned}
$$

Proof. (1) Since $B^{\prime \prime}>0$, the support of $V$ does not contain any of the singularities $x_{i}$ $(i=1, \ldots, N)$, and $\psi_{\Gamma}$ and $\phi_{\Gamma, \infty}$ are uniformly bounded on the union of the support of $V$ and $\Omega_{+}$(cf. 2.16) and (2.21) $)$, the existence and uniqueness of $\omega_{\Gamma, V} \in H_{0}^{1}(\Omega)$ that satisfies (4.37) follows from the Lax-Milgram Lemma 22, 25].

(2) Choosing $\eta \in C_{\mathrm{c}}^{1}(\Omega)$ in (4.37) with $\operatorname{supp}(\eta) \subset \Omega_{-}$and applying the Divergence Theorem, we obtain the first equation of 4.38 in a.e. $\Omega_{-}$. Since the right-hand side of this first equation is in $L^{2}\left(\Omega_{-}\right) \cap C\left(\Omega_{-}\right)$, it follows from the regularity theory [25, 32] that, with a possible modification of the value of $\omega_{\Gamma, V}$ on a set of zero Lebesgue measure, $\omega_{\Gamma, V}^{-} \in$ $H^{2}\left(\Omega_{-}\right) \cap C^{1}\left(\Omega_{-}\right)$. Now, the first equation in 4.38 holds for each point in $\Omega_{-}$. The second equation is similar to that in (4.28) (cf. (4.32)). By similar arguments, we obtain that $\omega_{\Gamma, V}^{+} \in H^{2}\left(\Omega_{+}\right) \cap C^{1}\left(\Omega_{+}\right)$and 4.39 . By splitting each of those two integrals in (4.37) that has the term $\nabla \eta$ into integrals over $\Omega_{-}$and $\Omega_{+}$, respectively, using the Divergence Theorem, and using (4.38) and 4.39), we obtain 4.40).

(3) Let $\phi_{\mathrm{C}}$ be given as in (1.6) and $t \in \mathbb{R}$. Denote

$$
\psi_{\mathrm{r}}=\psi_{\Gamma}-\hat{\phi}_{\mathrm{C}} \quad \text { and } \quad \psi_{\mathrm{r}, t}=\psi_{\Gamma_{t}}-\hat{\phi}_{\mathrm{C}}
$$

We first prove 4.41). By 4.25 (with $\hat{\phi}=\hat{\phi}_{\mathrm{C}}$ ) in Lemma 4.2, it suffices to prove that

$$
\lim _{t \rightarrow 0}\left\|\psi_{\mathrm{r}, t} \circ T_{t}-\psi_{\mathrm{r}}\right\|_{H^{1}(\Omega)}=0 .
$$

By Definition 2.1 and (2.3) (cf. also (2.12), we have

$$
\begin{aligned}
& \int_{\Omega}\left[\varepsilon_{\Gamma} \nabla \psi_{r} \cdot \nabla \eta+\chi_{+} B^{\prime}\left(\psi_{r}+\hat{\phi}_{\mathrm{C}}-\frac{\phi_{\Gamma, \infty}}{2}\right) \eta\right] d x \\
& \quad=-\left(\varepsilon_{+}-\varepsilon_{-}\right) \int_{\Omega_{+}} \nabla \hat{\phi}_{\mathrm{C}} \cdot \nabla \eta d x \quad \forall \eta \in H_{0}^{1}(\Omega) .
\end{aligned}
$$

Replacing $\Gamma, \Omega_{+}, \psi$, and $\eta$ in 4.44 by $\Gamma_{t}=T_{t}(\Gamma), T_{t}\left(\Omega_{+}\right), \psi_{\Gamma_{t}}$, and $\eta=\eta \circ T_{t}^{-1}$, respectively, we obtain by the change of variable $x=T_{t}(X)$ and (4.4) that

$$
\int_{\Omega}\left[\varepsilon_{\Gamma} A_{V}(t) \nabla\left(\psi_{r, t} \circ T_{t}\right) \cdot \nabla \eta+\chi_{+} B^{\prime}\left(\left(\psi_{\mathrm{r}, t}+\hat{\phi}_{\mathrm{C}}-\frac{\phi_{\Gamma_{t}, \infty}}{2}\right) \circ T_{t}\right) J_{t} \eta\right] d X
$$




$$
=-\left(\varepsilon_{+}-\varepsilon_{-}\right) \int_{\Omega_{+}} A_{V}(t) \nabla\left(\hat{\phi}_{\mathrm{C}} \circ T_{t}\right) \cdot \nabla \eta d X \quad \forall \eta \in H_{0}^{1}(\Omega) .
$$

Subtracting 4.44 from 4.45) and rearranging terms, we get

$$
\begin{aligned}
\int_{\Omega} \varepsilon_{\Gamma} & {\left[\nabla\left(\psi_{\mathrm{r}, t} \circ T_{t}\right)-\nabla \psi_{\mathrm{r}}\right] \cdot \nabla \eta d X } \\
=- & \int_{\Omega} \varepsilon_{\Gamma}\left[A_{V}(t)-I\right] \nabla\left(\psi_{\mathrm{r}, t} \circ T_{t}\right) \cdot \nabla \eta d X \\
& -\int_{\Omega_{+}} B^{\prime}\left(\left(\psi_{\mathrm{r}, t}+\hat{\phi}_{\mathrm{C}}-\frac{\phi_{\Gamma_{t}, \infty}}{2}\right) \circ T_{t}\right)\left(J_{t}-1\right) \eta d X \\
& -\int_{\Omega_{+}}\left[B^{\prime}\left(\left(\psi_{\mathrm{r}, t}+\hat{\phi}_{\mathrm{C}}-\frac{\phi_{\Gamma_{t}, \infty}}{2}\right) \circ T_{t}\right)-B^{\prime}\left(\psi_{\mathrm{r}}+\hat{\phi}_{\mathrm{C}}-\frac{\phi_{\Gamma, \infty}}{2}\right)\right] \eta d X \\
& -\left(\varepsilon_{+}-\varepsilon_{-}\right) \int_{\Omega_{+}}\left[\nabla\left(\hat{\phi}_{\mathrm{C}} \circ T_{t}\right)-\nabla \hat{\phi}_{\mathrm{C}}\right] \cdot \nabla \eta d X \\
& -\left(\varepsilon_{+}-\varepsilon_{-}\right) \int_{\Omega_{+}}\left[A_{V}(t)-I\right] \nabla\left(\hat{\phi}_{\mathrm{C}} \circ T_{t}\right) \cdot \nabla \eta d X \quad \forall \eta \in H_{0}^{1}(\Omega) .
\end{aligned}
$$

Setting $\eta=\psi_{\mathrm{r}, t} \circ T_{t}-\psi_{\mathrm{r}}$, we have by the uniform bound of all $\psi_{\mathrm{r}, t}$ and $\phi_{\Gamma_{t}, \infty}$ (cf. (2.21) and (2.7)), the Mean-Value Theorem, and the convexity of $B$ that

$$
\begin{aligned}
- & {\left[B^{\prime}\left(\left(\psi_{\mathrm{r}, t}+\hat{\phi}_{\mathrm{C}}-\frac{\phi_{\Gamma_{t}, \infty}}{2}\right) \circ T_{t}\right)-B^{\prime}\left(\psi_{\mathrm{r}}+\hat{\phi}_{\mathrm{C}}-\frac{\phi_{\Gamma, \infty}}{2}\right)\right] \eta } \\
= & -B^{\prime \prime}\left(\lambda_{t}\right)\left(\psi_{\mathrm{r}, t} \circ T_{t}-\psi_{\mathrm{r}}+\hat{\phi}_{\mathrm{C}} \circ T_{t}-\hat{\phi}_{\mathrm{C}}+\frac{1}{2} \phi_{\Gamma_{t}, \infty} \circ T_{t}-\frac{1}{2} \phi_{\Gamma, \infty}\right)\left(\psi_{\mathrm{r}, t} \circ T_{t}-\psi_{\mathrm{r}}\right) \\
= & -B^{\prime \prime}\left(\lambda_{t}\right)\left(\psi_{\mathrm{r}, t} \circ T_{t}-\psi_{\mathrm{r}}\right)^{2} \\
& -B^{\prime \prime}\left(\lambda_{t}\right)\left(\hat{\phi}_{\mathrm{C}} \circ T_{t}-\hat{\phi}_{\mathrm{C}}+\frac{1}{2} \phi_{\Gamma_{t}, \infty} \circ T_{t}-\frac{1}{2} \phi_{\Gamma, \infty}\right)\left(\psi_{\mathrm{r}, t} \circ T_{t}-\psi_{\mathrm{r}}\right) \\
\leq & C\left|\left(\hat{\phi}_{\mathrm{C}} \circ T_{t}-\hat{\phi}_{\mathrm{C}}\right)\left(\psi_{\mathrm{r}, t} \circ T_{t}-\psi_{\mathrm{r}}\right)\right|+C\left|\left(\phi_{\Gamma_{t}, \infty} \circ T_{t}-\phi_{\Gamma, \infty}\right)\left(\psi_{\mathrm{r}, t} \circ T_{t}-\psi_{\mathrm{r}}\right)\right|
\end{aligned}
$$

where $\lambda_{t}$ is in between $\left(\psi_{\mathrm{r}, t}+\hat{\phi}_{\mathrm{C}}-\phi_{\Gamma_{t}, \infty} / 2\right) \circ T_{t}$ and $\psi_{\mathrm{r}}+\hat{\phi}_{\mathrm{C}}-\phi_{\Gamma, \infty} / 2$ at each point in $\Omega_{+}$, and the constant $C>0$ is independent of $t$ and $\Gamma$. Now, the combination of (4.46) with $\eta=\psi_{\mathrm{r}, t} \circ T_{t}-\psi_{\mathrm{r}}$ and (4.47), together with the uniform bounds for $\psi_{\mathrm{r}, t}$ and $\phi_{\Gamma_{t}, \infty}$, and the Cauchy-Schwarz and Poincaré inequalities, leads to

$$
\begin{aligned}
\left\|\psi_{\mathrm{r}, t} \circ T_{t}-\psi_{\mathrm{r}}\right\|_{H^{1}(\Omega)} \leq & C\left\|A_{V}(t)-I\right\|_{L^{\infty}(\Omega)}\left\|\psi_{\mathrm{r}, t} \circ T_{t}\right\|_{H^{1}(\Omega)}+C\left\|J_{t}-1\right\|_{L^{\infty}(\Omega)} \\
& +C\left\|\hat{\phi}_{\mathrm{C}} \circ T_{t}-\hat{\phi}_{\mathrm{C}}\right\|_{H^{1}\left(\Omega_{+}\right)}+C\left\|\phi_{\Gamma_{t}, \infty} \circ T_{t}-\phi_{\Gamma, \infty}\right\|_{H^{1}(\Omega)} \\
& +C\left\|A_{V}(t)-I\right\|_{L^{\infty}\left(\Omega_{+}\right)}\left\|\hat{\phi}_{\mathrm{C}} \circ T_{t}\right\|_{H^{1}\left(\Omega_{+}\right)} .
\end{aligned}
$$

Now the convergence (4.43) follows from (4.2), (4.3), (4.7), (4.8), the uniform bound of $\psi_{\mathrm{r}, t}$, Lemma 4.2 (with $\hat{\phi}=\hat{\phi}_{\mathrm{C}}$ ), and Lemma 4.3 . 
We now prove 4.42). Let us denote $\hat{\omega}_{\Gamma, V}=\omega_{\Gamma, V}-\nabla \hat{\phi}_{\mathrm{C}} \cdot V$. By Lemma 4.2 (cf. 4.26) with $\hat{\phi}=\hat{\phi}_{\mathrm{C}}$ ), we need only to prove that

$$
\lim _{t \rightarrow 0}\left\|\frac{\psi_{\mathrm{r}, t} \circ T_{t}-\psi_{\mathrm{r}}}{t}-\hat{\omega}_{\Gamma, V}\right\|_{H^{1}(\Omega)}=0 .
$$

We first note that the Divergence Theorem and the calculations in 4.32 imply that

$$
\begin{aligned}
\int_{\Omega} & {\left[A_{V}^{\prime}(0) \nabla \hat{\phi}_{\mathrm{C}}+\nabla\left(\nabla \hat{\phi}_{\mathrm{C}} \cdot V\right)\right] \cdot \nabla \eta d x } \\
& =-\int_{\Omega}\left[\nabla \cdot\left(A_{V}^{\prime}(0) \nabla \hat{\phi}_{\mathrm{C}}\right)+\Delta\left(\nabla \hat{\phi}_{\mathrm{C}} \cdot V\right)\right] \eta d x \\
& =0 \quad \forall \eta \in H_{0}^{1}(\Omega) .
\end{aligned}
$$

This allows us to rewrite 4.37) into the following equation for $\hat{\omega}_{\Gamma, V}$ :

$$
\begin{aligned}
\int_{\Omega}\left[\varepsilon_{\Gamma} \nabla \hat{\omega}_{\Gamma, V} \cdot \nabla \eta+\chi_{+} B^{\prime \prime}\left(\psi_{\mathrm{r}}+\hat{\phi}_{\mathrm{C}}-\frac{\phi_{\Gamma, \infty}}{2}\right) \hat{\omega}_{\Gamma, V} \eta\right] d X \\
=-\int_{\Omega} \varepsilon_{\Gamma} A_{V}^{\prime}(0) \nabla\left(\psi_{\Gamma}-\hat{\phi}_{\mathrm{C}}\right) \cdot \nabla \eta d X \\
\quad-\int_{\Omega_{+}}\left[B^{\prime}\left(\psi_{\mathrm{r}}+\hat{\phi}_{\mathrm{C}}-\frac{\phi_{\Gamma, \infty}}{2}\right)(\nabla \cdot V)\right. \\
\left.\quad+B^{\prime \prime}\left(\psi_{\mathrm{r}}+\hat{\phi}_{\mathrm{C}}-\frac{\phi_{\Gamma, \infty}}{2}\right)\left(\nabla \hat{\phi}_{\mathrm{C}} \cdot V-\frac{\zeta_{\Gamma, V}}{2}\right)\right] \eta d X \quad \\
\quad-\left(\varepsilon_{+}-\varepsilon_{-}\right) \int_{\Omega_{+}}\left[A_{V}^{\prime}(0) \nabla \hat{\phi}_{\mathrm{C}}+\nabla\left(\nabla \hat{\phi}_{\mathrm{C}} \cdot V\right)\right] \cdot \nabla \eta d X \quad \forall \eta \in H_{0}^{1}(\Omega) .
\end{aligned}
$$

Multiplying both sides of (4.46) by $1 / t$ and combining the resulting equation with (4.49), we obtain by rearranging terms that

$$
\begin{aligned}
\int_{\Omega} \varepsilon_{\Gamma} & \nabla \\
=- & \int_{\Omega} \varepsilon_{\Gamma}\left[\left(\frac{A_{\mathrm{r}, t} \circ T_{t}-\psi_{\mathrm{r}}}{t}-\hat{\omega}_{\Gamma, V}\right) \cdot \nabla \eta d X\right. \\
- & \int_{\Omega_{+}}\left[B^{\prime}\left(\left(\psi_{\mathrm{r}, t}+\hat{\phi}_{\mathrm{C}}-\frac{\phi_{\Gamma t, \infty}}{2}\right) \circ T_{t}\right)\left(\frac{J_{t}-1}{t}\right)\right. \\
& \left.-B^{\prime}\left(\psi_{\mathrm{r}}+\hat{\phi}_{\mathrm{C}}-\frac{\phi_{\Gamma, \infty}}{2}\right)(\nabla \cdot V)\right] \eta d X \\
- & \int_{\Omega_{+}}\left\{\frac{1}{t}\left[B^{\prime}\left(\left(\psi_{\mathrm{r}, t}+\hat{\phi}_{\mathrm{C}}-\frac{\phi_{\Gamma t, \infty}}{2}\right) \circ T_{t}\right)-B^{\prime}\left(\psi_{\mathrm{r}}+\hat{\phi}_{\mathrm{C}}-\frac{\phi_{\Gamma, \infty}}{2}\right)\right]\right. \\
& \left.-B^{\prime \prime}\left(\psi_{\mathrm{r}}+\hat{\phi}_{\mathrm{C}}-\frac{\phi_{\Gamma, \infty}}{2}\right)\left(\hat{\omega}_{\Gamma, V}+\nabla \hat{\phi}_{\mathrm{C}} \cdot V-\frac{\zeta_{\Gamma, V}}{2}\right)\right\} \eta d X
\end{aligned}
$$




$$
\begin{aligned}
& -\left(\varepsilon_{+}-\varepsilon_{-}\right) \int_{\Omega_{+}}\left[\left(\frac{A_{V}(t)-I}{t}\right) \nabla\left(\hat{\phi}_{\mathrm{C}} \circ T_{t}\right)-A_{V}^{\prime}(0) \nabla \hat{\phi}_{\mathrm{C}}\right] \cdot \nabla \eta d X \\
& -\left(\varepsilon_{+}-\varepsilon_{-}\right) \int_{\Omega_{+}} \nabla\left(\frac{\hat{\phi}_{\mathrm{C}} \circ T_{t}-\hat{\phi}_{\mathrm{C}}}{t}-\nabla \hat{\phi}_{\mathrm{C}} \cdot V\right) \cdot \nabla \eta d X \quad \forall \eta \in H_{0}^{1}(\Omega) .
\end{aligned}
$$

Specifying $\eta=\left(\psi_{\mathrm{r}, t} \circ T_{t}-\psi_{\mathrm{r}}\right) / t-\hat{\omega}_{\Gamma, V} \in H_{0}^{1}(\Omega)$, we have by the fact that $B^{\prime \prime}>0$, the Mean-Value Theorem, the uniform bound for all the functions $\psi_{\mathrm{r}, t}, \zeta_{\Gamma, V}$, and $\omega_{\Gamma, V}$ (cf. (2.7), (2.16), (2.21) ) that in $\Omega_{+}$

$$
\begin{aligned}
& -\left\{\frac{1}{t}\left[B^{\prime}\left(\left(\psi_{\mathrm{r}, t}+\hat{\phi}_{\mathrm{C}}-\frac{\phi_{\Gamma_{t}, \infty}}{2}\right) \circ T_{t}\right)-B^{\prime}\left(\psi_{\mathrm{r}}+\hat{\phi}_{\mathrm{C}}-\frac{\phi_{\Gamma, \infty}}{2}\right)\right]\right. \\
& \left.-B^{\prime \prime}\left(\psi_{\mathrm{r}}+\hat{\phi}_{\mathrm{C}}-\frac{\phi_{\Gamma, \infty}}{2}\right)\left(\hat{\omega}_{\Gamma, V}+\nabla \hat{\phi}_{\mathrm{C}} \cdot V-\frac{\zeta_{\Gamma, V}}{2}\right)\right\}\left(\frac{\psi_{\mathrm{r}, t} \circ T_{t}-\psi_{\mathrm{r}}}{t}-\hat{\omega}_{\Gamma, V}\right) \\
& =-B^{\prime \prime}\left(\xi_{t}\right)\left(\frac{\psi_{\mathrm{r}, t} \circ T_{t}-\psi_{\mathrm{r}}}{t}+\frac{\hat{\phi}_{\mathrm{C}} \circ T_{t}-\hat{\phi}_{\mathrm{C}}}{t}-\frac{\phi_{\Gamma t, \infty} \circ T_{t}-\phi_{\Gamma, \infty}}{2 t}\right)\left(\frac{\psi_{\mathrm{r}, t} \circ T_{t}-\psi_{\mathrm{r}}}{t}-\hat{\omega}_{\Gamma, V}\right) \\
& +B^{\prime \prime}\left(\psi_{\mathrm{r}}+\hat{\phi}_{\mathrm{C}}-\frac{\phi_{\Gamma, \infty}}{2}\right)\left(\hat{\omega}_{\Gamma, V}+\nabla \hat{\phi}_{\mathrm{C}} \cdot V-\frac{\zeta_{\Gamma, V}}{2}\right)\left(\frac{\psi_{\mathrm{r}, t} \circ T_{t}-\psi_{\mathrm{r}}}{t}-\hat{\omega}_{\Gamma, V}\right) \\
& =-B^{\prime \prime}\left(\xi_{t}\right)\left(\frac{\psi_{\mathrm{r}, t} \circ T_{t}-\psi_{\mathrm{r}}}{t}-\hat{\omega}_{\Gamma, V}\right)^{2} \\
& -B^{\prime \prime}\left(\xi_{t}\right)\left(\hat{\omega}_{\Gamma, V}+\frac{\hat{\phi}_{\mathrm{C}} \circ T_{t}-\hat{\phi}_{\mathrm{C}}}{t}-\frac{\phi_{\Gamma t, \infty} \circ T_{t}-\phi_{\Gamma, \infty}}{2 t}\right)\left(\frac{\psi_{\mathrm{r}, t} \circ T_{t}-\psi_{\mathrm{r}}}{t}-\hat{\omega}_{\Gamma, V}\right) \\
& +\left[B^{\prime \prime}\left(\psi_{\mathrm{r}}+\hat{\phi}_{\mathrm{C}}-\frac{\phi_{\Gamma, \infty}}{2}\right)-B^{\prime \prime}\left(\xi_{t}\right)\right]\left(\hat{\omega}_{\Gamma, V}+\nabla \hat{\phi}_{\mathrm{C}} \cdot V-\frac{\zeta_{\Gamma, V}}{2}\right)\left(\frac{\psi_{\mathrm{r}, t} \circ T_{t}-\psi_{\mathrm{r}}}{t}-\hat{\omega}_{\Gamma, V}\right) \\
& +B^{\prime \prime}\left(\xi_{t}\right)\left(\hat{\omega}_{\Gamma, V}+\nabla \hat{\phi}_{\mathrm{C}} \cdot V-\frac{\zeta_{\Gamma, V}}{2}\right)\left(\frac{\psi_{\mathrm{r}, t} \circ T_{t}-\psi_{\mathrm{r}}}{t}-\hat{\omega}_{\Gamma, V}\right) \\
& \leq-B^{\prime \prime}\left(\xi_{t}\right)\left(\frac{\hat{\phi}_{\mathrm{C}} \circ T_{t}-\hat{\phi}_{\mathrm{C}}}{t}-\nabla \hat{\phi}_{\mathrm{C}} \cdot V-\frac{\phi_{\Gamma t, \infty} \circ T_{t}-\phi_{\Gamma, \infty}}{2 t}+\frac{\zeta_{\Gamma, V}}{2}\right) \\
& \cdot\left(\frac{\psi_{\mathrm{r}, t} \circ T_{t}-\psi_{\mathrm{r}}}{t}-\hat{\omega}_{\Gamma, V}\right) \\
& +B^{\prime \prime \prime}\left(\sigma_{t}\right)\left(\psi_{\mathrm{r}}+\hat{\phi}_{\mathrm{C}}-\frac{\phi_{\Gamma, \infty}}{2}-\xi_{t}\right)\left(\hat{\omega}_{\Gamma, V}+\nabla \hat{\phi}_{\mathrm{C}} \cdot V-\frac{\zeta_{\Gamma, V}}{2}\right)\left(\frac{\psi_{\mathrm{r}, t} \circ T_{t}-\psi_{\mathrm{r}}}{t}-\hat{\omega}_{\Gamma, V}\right) \\
& \leq C\left(\left|\frac{\hat{\phi}_{\mathrm{C}} \circ T_{t}-\hat{\phi}_{\mathrm{C}}}{t}-\nabla \hat{\phi}_{\mathrm{C}} \cdot V\right|+\left|\frac{\phi_{\Gamma_{t}, \infty} \circ T_{t}-\phi_{\Gamma, \infty}}{t}-\zeta_{\Gamma, V}\right|\right)\left|\frac{\psi_{\mathrm{r}, t} \circ T_{t}-\psi_{\mathrm{r}}}{t}-\hat{\omega}_{\Gamma, V}\right| \\
& +C\left(\left|\psi_{\mathrm{r}, t} \circ T_{t}-\psi_{\mathrm{r}}\right|+\left|\hat{\phi}_{\mathrm{C}} \circ T_{t}-\hat{\phi}_{\mathrm{C}}\right|+\left|\phi_{\Gamma_{t}, \infty} \circ T_{t}-\phi_{\Gamma, \infty}\right|\right)\left|\frac{\psi_{\mathrm{r}, t} \circ T_{t}-\psi_{\mathrm{r}}}{t}-\hat{\omega}_{\Gamma, V}\right|,
\end{aligned}
$$

where $\xi_{t}$ and $\sigma_{t}$ are in between $\left(\psi_{\mathrm{r}, t}+\hat{\phi}_{\mathrm{C}}-\phi_{\Gamma_{t}, \infty} / 2\right) \circ T_{t}$ and $\psi_{\mathrm{r}}+\hat{\phi}_{\mathrm{C}}-\phi_{\Gamma, \infty} / 2$ at each point in $\Omega_{+}$. Now, combining this inequality and the identity (4.50) with $\eta=\left(\psi_{\mathrm{r}, t} \circ T_{t}-\psi_{\mathrm{r}}\right) / t-\hat{\omega}_{\Gamma, V} \in$ 
$H_{0}^{1}(\Omega)$, we obtain by the Poincaré and Cauchy-Schwarz inequalities and rearranging terms that

$$
\begin{aligned}
& \left\|\frac{\psi_{\mathrm{r}, t} \circ T_{t}-\psi_{\mathrm{r}}}{t}-\hat{\omega}_{\Gamma, V}\right\|_{H^{1}(\Omega)}^{2} \\
& \leq C \int_{\Omega}\left|\left(\frac{A_{V}(t)-I}{t}\right) \nabla\left(\psi_{\mathrm{r}, t} \circ T_{t}\right)-A_{V}^{\prime}(0) \nabla \psi_{\mathrm{r}}\right|^{2} d X \\
& +C \int_{\Omega_{+}} \mid B^{\prime}\left(\left(\psi_{\mathrm{r}, t}+\hat{\phi}_{\mathrm{C}}-\frac{\phi_{\Gamma_{t}, \infty}}{2}\right) \circ T_{t}\right)\left(\frac{J_{t}-1}{t}\right) \\
& -\left.B^{\prime}\left(\psi_{\mathrm{r}}+\hat{\phi}_{\mathrm{C}}-\frac{\phi_{\Gamma, \infty}}{2}\right)(\nabla \cdot V)\right|^{2} d X \\
& +C\left(\left\|\frac{\hat{\phi}_{\mathrm{C}} \circ T_{t}-\hat{\phi}_{\mathrm{C}}}{t}-\nabla \hat{\phi}_{\mathrm{C}} \cdot V\right\|_{H^{1}\left(\Omega_{+}\right)}^{2}+\left\|\frac{\phi_{\Gamma_{t}, \infty} \circ T_{t}-\phi_{\Gamma, \infty}}{t}-\zeta_{\Gamma, V}\right\|_{L^{2}\left(\Omega_{+}\right)}^{2}\right. \\
& \left.+\left\|\psi_{\mathrm{r}, t} \circ T_{t}-\psi_{\mathrm{r}}\right\|_{L^{2}\left(\Omega_{+}\right)}^{2}+\left\|\hat{\phi}_{\mathrm{C}} \circ T_{t}-\hat{\phi}_{\mathrm{C}}\right\|_{L^{2}\left(\Omega_{+}\right)}^{2}+\left\|\phi_{\Gamma_{t}, \infty} \circ T_{t}-\phi_{\Gamma, \infty}\right\|_{L^{2}\left(\Omega_{+}\right)}^{2}\right) \\
& +C \int_{\Omega_{+}}\left|\left(\frac{A_{V}(t)-I}{t}\right) \nabla\left(\hat{\phi}_{\mathrm{C}} \circ T_{t}\right)-A_{V}^{\prime}(0) \nabla \hat{\phi}_{\mathrm{C}}\right|^{2} d X \\
& =C\left[S_{1}(t)+S_{2}(t)+S_{3}(t)+S_{4}(t)\right] .
\end{aligned}
$$

It follows from 4.6- 4.8 , Lemma 4.2 (with $\hat{\phi}=\hat{\phi}_{\mathrm{C}}$ ), and 4.41) that

$$
\begin{aligned}
S_{1}(t) & =\int_{\Omega}\left|\left[\frac{A_{V}(t)-I}{t}\right] \nabla\left(\psi_{\mathrm{r}, t} \circ T_{t}\right)-A_{V}^{\prime}(0) \nabla \psi_{\mathrm{r}}\right|^{2} d X \\
& \leq 2 \int_{\Omega}\left|\left[\frac{A_{V}(t)-I}{t}-A_{V}^{\prime}(0)\right] \nabla\left(\psi_{\mathrm{r}, t} \circ T_{t}\right)\right|^{2} d X+2 \int_{\Omega}\left|A_{V}^{\prime}(0) \nabla\left(\psi_{\mathrm{r}, t} \circ T_{t}-\psi_{\mathrm{r}}\right)\right|^{2} d X \\
& \rightarrow 0 \quad \text { as } t \rightarrow 0 .
\end{aligned}
$$

By the uniform boundedness of $\psi_{\mathrm{r}, t}$ and $\phi_{\Gamma_{t}, \infty}$ (cf. (2.7), (2.16), 2.21) $)$ the Mean-Value Theorem, 4.2) and (4.3), Lemmas 4.2 and 4.3, and 4.41), we have

$$
\begin{aligned}
S_{2}(t)= & \int_{\Omega_{+}}\left|B^{\prime}\left(\left(\psi_{\mathrm{r}, t}+\hat{\phi}_{\mathrm{C}}-\frac{\phi_{\Gamma_{t}, \infty}}{2}\right) \circ T_{t}\right)\left(\frac{J_{t}-1}{t}\right)-B^{\prime}\left(\psi_{\mathrm{r}}+\hat{\phi}_{\mathrm{C}}-\frac{\phi_{\Gamma, \infty}}{2}\right)(\nabla \cdot V)\right|^{2} d X \\
\leq & 2 \int_{\Omega_{+}}\left|B^{\prime}\left(\left(\psi_{\mathrm{r}, t}+\hat{\phi}_{\mathrm{C}}-\frac{\phi_{\Gamma_{t}, \infty}}{2}\right) \circ T_{t}\right)\left(\frac{J_{t}-1}{t}-\nabla \cdot V\right)\right|^{2} d X \\
& +2 \int_{\Omega_{+}}\left|B^{\prime}\left(\left(\psi_{\mathrm{r}, t}+\hat{\phi}_{\mathrm{C}}-\frac{\phi_{\Gamma_{t}, \infty}}{2}\right) \circ T_{t}\right)-B^{\prime}\left(\psi_{\mathrm{r}}+\hat{\phi}_{\mathrm{C}}-\frac{\phi_{\Gamma, \infty}}{2}\right)\right|^{2}|\nabla \cdot V|^{2} d X \\
\leq & C \int_{\Omega_{+}}\left|\frac{J_{t}-1}{t}-\nabla \cdot V\right|^{2} d X
\end{aligned}
$$




$$
\begin{aligned}
& +C \int_{\Omega_{+}}\left(\left|\psi_{\mathrm{r}, t} \circ T_{t}-\psi_{\mathrm{r}}\right|^{2}+\left|\hat{\phi}_{\mathrm{C}} \circ T_{t}-\hat{\phi}_{\mathrm{C}}\right|^{2}+\left|\phi_{\Gamma_{t}, \infty} \circ T_{t}-\phi_{\Gamma, \infty}\right|^{2}\right) d X \\
& \rightarrow 0 \quad \text { as } t \rightarrow 0 .
\end{aligned}
$$

By Lemma 4.2 (with $\hat{\phi}=\hat{\phi}_{\mathrm{C}}$ ), Lemma 4.3, and 4.41, we have

$$
\begin{aligned}
S_{3}(t)= & \left\|\frac{\hat{\phi}_{\mathrm{C}} \circ T_{t}-\hat{\phi}_{\mathrm{C}}}{t}-\nabla \hat{\phi}_{\mathrm{C}} \cdot V\right\|_{H^{1}\left(\Omega_{+}\right)}^{2}+\left\|\frac{\phi_{\Gamma_{t}, \infty} \circ T_{t}-\phi_{\Gamma, \infty}}{t}-\zeta_{\Gamma, V}\right\|_{L^{2}\left(\Omega_{+}\right)} \\
& +\left\|\psi_{\mathrm{r}, t} \circ T_{t}-\psi_{\mathrm{r}}\right\|_{L^{2}\left(\Omega_{+}\right)}+\left\|\hat{\phi}_{\mathrm{C}} \circ T_{t}-\hat{\phi}_{\mathrm{C}}\right\|_{L^{2}\left(\Omega_{+}\right)}^{2}+\left\|\phi_{\Gamma_{t}, \infty} \circ T_{t}-\phi_{\Gamma, \infty}\right\|_{L^{2}\left(\Omega_{+}\right)}^{2} \\
& \rightarrow 0 \quad \text { as } t \rightarrow 0 .
\end{aligned}
$$

It follows from 4.6-4.8 and Lemma 4.2 (with $\left.\hat{\phi}=\hat{\phi}_{\mathrm{C}}\right)$ that

$$
\begin{aligned}
S_{4}(t) & =\int_{\Omega_{+}}\left|\left[\frac{A_{V}(t)-I}{t}\right] \nabla\left(\hat{\phi}_{\mathrm{C}} \circ T_{t}\right)-A_{V}^{\prime}(0) \nabla \hat{\phi}_{\mathrm{C}}\right|^{2} d X \\
& \leq C \int_{\Omega_{+}}\left|\left[\frac{A_{V}(t)-I}{t}-A_{V}^{\prime}(0)\right] \nabla\left(\hat{\phi}_{\mathrm{C}} \circ T_{t}\right)\right|^{2}+C \int_{\Omega_{+}}\left|\nabla\left(\hat{\phi}_{\mathrm{C}} \circ T_{t}-\hat{\phi}_{\mathrm{C}}\right)\right|^{2} d X \\
& \rightarrow 0 \quad \text { as } t \rightarrow 0 .
\end{aligned}
$$

Now the desired convergence 4.48) follows from 4.51-4.55).

\section{Proof of Theorem 3.2}

Proof of Theorem 3.2. Fix $V \in \mathcal{V}\left(\mathrm{cf}\right.$. (3.6)). Let $\left\{T_{t}\right\}_{t \in \mathbb{R}}$ be the family of diffeomorphisms from $\mathbb{R}^{3}$ to $\mathbb{R}^{3}$ defined by $T_{t}(X)=x(t, X)$ as the solution to the initial-value problem (3.7). We proceed in five steps. In Step 1, we calculate the limit as $t \rightarrow 0$ that defines the variation $\delta_{\Gamma, V} E[\Gamma]$; cf. Definition 3.1. In Step 2, we simplify the expression of $\delta_{\Gamma, V} E[\Gamma]$. In Step 3, we convert all the volume integrals in $\delta_{\Gamma, V} E[\Gamma]$ into surface integrals on the boundary $\Gamma$, except one volume integral that involves the $B^{\prime}$ term. In Step 4, we rewrite the surface integrals to have the desired form (i.e., with a factor $V \cdot n$ in the integrand). Finally, in Step 5, we treat the only volume integral term that involves $B^{\prime}$ to get the desired formula.

Step 1. Let $t \in \mathbb{R}$. We recall that $\phi_{\Gamma_{t}, \infty}, \hat{\phi}_{\Gamma_{t}, \infty}$, and $\psi_{\Gamma_{t}}$ are the solutions to 2.5), 2.11, and 2.20) with $\Gamma_{t}=T_{t}(\Gamma)$ replacing $\Gamma$, respectively, and that all these functions have the boundary value $\phi_{\infty}$ on $\partial \Omega$. Recall that $\hat{\phi}_{0}$ and $\hat{\phi}_{\infty}$ are defined by 2.3) and 2.4). We denote in this proof

$$
\psi_{\mathrm{r}}=\psi_{\Gamma}-\hat{\phi}_{\Gamma, \infty} \quad \text { and } \quad \psi_{\mathrm{r}, t}=\psi_{\Gamma_{t}}-\hat{\phi}_{\Gamma_{t}, \infty}
$$

By (3.1) and (3.2) with $\Gamma_{t}$ replacing $\Gamma$, the definition of $A_{V}(t)$ (4.4) and $J_{t}$ (4.1), and the change of variable $x=T_{t}(X)$, we have

$$
E\left[\Gamma_{t}\right]=-\int_{\Omega} \frac{\varepsilon_{\Gamma_{t}}}{2}\left|\nabla \psi_{\mathrm{r}, t}\right|^{2} d x-\int_{T_{t}\left(\Omega_{+}\right)} B\left(\psi_{\Gamma_{t}}-\frac{\phi_{\Gamma_{t}, \infty}}{2}\right) d x
$$




$$
\begin{aligned}
& +\frac{\varepsilon_{-}-\varepsilon_{+}}{2} \int_{T_{t}\left(\Omega_{+}\right)} \nabla \hat{\phi}_{\Gamma_{t}, \infty} \cdot \nabla \hat{\phi}_{0} d x+W \\
= & -\int_{\Omega} \frac{\varepsilon_{\Gamma}}{2}\left[A_{V}(t) \nabla\left(\psi_{r, t} \circ T_{t}\right) \cdot \nabla\left(\psi_{r, t} \circ T_{t}\right)\right] d X \\
& -\int_{\Omega_{+}} B\left(\left(\psi_{\Gamma_{t}}-\frac{\phi_{\Gamma_{t}, \infty}}{2}\right) \circ T_{t}\right) J_{t} d X \\
& +\frac{\varepsilon_{-}-\varepsilon_{+}}{2} \int_{\Omega_{+}} A_{V}(t) \nabla\left(\hat{\phi}_{\Gamma_{t}, \infty} \circ T_{t}\right) \cdot \nabla\left(\hat{\phi}_{0} \circ T_{t}\right) d X+W,
\end{aligned}
$$

where $W=(1 / 2) \sum_{i=1}^{N} Q_{i}\left(\hat{\phi}_{\infty}-\hat{\phi}_{0}\right)\left(x_{i}\right)$ is independent of $\Gamma$. Consequently,

$$
\begin{aligned}
\frac{E\left[\Gamma_{t}\right]-E[\Gamma]}{t}=- & \int_{\Omega} \frac{\varepsilon_{\Gamma}}{2 t}\left[A_{V}(t) \nabla\left(\psi_{\mathrm{r}, t} \circ T_{t}\right) \cdot \nabla\left(\psi_{\mathrm{r}, t} \circ T_{t}\right)-\nabla \psi_{\mathrm{r}} \cdot \nabla \psi_{\mathrm{r}}\right] d X \\
& -\int_{\Omega_{+}} \frac{1}{t}\left[B\left(\left(\psi_{\Gamma_{t}}-\frac{\phi_{\Gamma_{t}, \infty}}{2}\right) \circ T_{t}\right) J_{t}-B\left(\psi_{\Gamma}-\frac{\phi_{\Gamma, \infty}}{2}\right)\right] d X \\
& +\frac{\varepsilon_{-}-\varepsilon_{+}}{2} \int_{\Omega_{+}} \frac{1}{t}\left[A_{V}(t) \nabla\left(\hat{\phi}_{\Gamma_{t}, \infty} \circ T_{t}\right) \cdot \nabla\left(\hat{\phi}_{0} \circ T_{t}\right)-\nabla \hat{\phi}_{\Gamma, \infty} \cdot \nabla \hat{\phi}_{0}\right] d X \\
=- & \delta_{1}(t)-\delta_{2}(t)+\frac{\varepsilon_{-}-\varepsilon_{+}}{2} \delta_{3}(t) .
\end{aligned}
$$

By rearranging the terms, we obtain that

$$
\begin{aligned}
\delta_{1}(t)=\int_{\Omega} & \frac{\varepsilon_{\Gamma}}{2}\left[\frac{A_{V}(t)-I-t A_{V}^{\prime}(0)}{t}\right] \nabla\left(\psi_{\mathrm{r}, t} \circ T_{t}\right) \cdot \nabla\left(\psi_{\mathrm{r}, t} \circ T_{t}\right) d X \\
& +\int_{\Omega} \frac{\varepsilon_{\Gamma}}{2} A_{V}^{\prime}(0) \nabla\left(\psi_{\mathrm{r}, t} \circ T_{t}\right) \cdot \nabla\left(\psi_{\mathrm{r}, t} \circ T_{t}\right) d X \\
& +\int_{\Omega} \frac{\varepsilon_{\Gamma}}{2}\left[\nabla\left(\psi_{\mathrm{r}, t} \circ T_{t}\right)+\nabla \psi_{\mathrm{r}}\right] \cdot \nabla\left(\frac{\psi_{\mathrm{r}, t} \circ T_{t}-\psi_{\mathrm{r}}}{t}\right) d X .
\end{aligned}
$$

It thus follows from 4.7, 4.8, Lemma 4.4, and Lemma 4.5 that

$$
\lim _{t \rightarrow 0} \delta_{1}(t)=\int_{\Omega} \varepsilon_{\Gamma}\left[\frac{1}{2} A_{V}^{\prime}(0) \nabla \psi_{\mathrm{r}} \cdot \nabla \psi_{\mathrm{r}}+\nabla \psi_{\mathrm{r}} \cdot \nabla\left(\omega_{\Gamma, V}-\xi_{\Gamma, V}\right)\right] d X,
$$

where $\xi_{\Gamma, V}$ and $\omega_{\Gamma, V}$ are defined in 4.35) in Lemma 4.4 and 4.37) in Lemma 4.5, respectively.

Denote $q=\psi_{\Gamma}-\phi_{\Gamma, \infty} / 2$ and $q_{t}=\left(\psi_{\Gamma_{t}}-\phi_{\Gamma_{t}, \infty} / 2\right) \circ T_{t}$. The second term $\delta_{2}(t)$ in (5.2) can be written as

$$
\delta_{2}(t)=\int_{\Omega_{+}} \frac{J_{t}-1}{t} B\left(q_{t}\right) d X+\int_{\Omega_{+}} \frac{B\left(q_{t}\right)-B(q)}{t} d X
$$

Since the $L^{\infty}(\Omega)$-norm of $q_{t}$ is bounded uniformly in $t \in \mathbb{R}(\operatorname{cf} .(2.15)$ and $(2.21)$ ), it follows from Lemma 4.3 and Lemma 4.5 that $q_{t} \rightarrow q$ in $L^{2}(\Omega)$. Hence, $B\left(q_{t}\right) \rightarrow B(q)$ in $L^{2}\left(\Omega_{+}\right)$as 
$t \rightarrow 0$. This, together with (4.2) and (4.3), implies that

$$
\lim _{t \rightarrow 0} \int_{\Omega_{+}} \frac{J_{t}-1}{t} B\left(q_{t}\right) d X=\int_{\Omega_{+}}(\nabla \cdot V) B(q) d X .
$$

Now Taylor's expansion implies that

$$
\begin{aligned}
& \frac{B\left(q_{t}(X)\right)-B(q(X))}{t} \\
& \quad=B^{\prime}(q(X)) \frac{q_{t}(X)-q(X)}{t}+\frac{1}{2} B^{\prime \prime}\left(\eta_{t}(X)\right)\left[q_{t}(X)-q(X)\right] \frac{q_{t}(X)-q(X)}{t}, \quad \text { a.e. } X \in \Omega_{+},
\end{aligned}
$$

where $\eta_{t}(X)$ is in between $q(X)$ and $q_{t}(X)$, and its $L^{\infty}(\Omega)$-norm is bounded uniformly in $t$. It then follows from Lemma 4.3 and Lemma 4.5 that

$$
\left|\int_{\Omega_{+}} B^{\prime \prime}\left(\eta_{t}\right)\left(q_{t}-q\right) \frac{q_{t}-q}{t} d X\right| \leq C\left\|q_{t}-q\right\|_{L^{2}\left(\Omega_{+}\right)}\left\|\frac{q_{t}-q}{t}\right\|_{L^{2}\left(\Omega_{+}\right)} \rightarrow 0 \quad \text { as } t \rightarrow 0,
$$

where $C$ is a constant independent of $t$. Consequently, by Lemma 4.3 and Lemma 4.5 that

$$
\lim _{t \rightarrow 0} \int_{\Omega_{+}} \frac{B\left(q_{t}\right)-B(q)}{t} d X=\lim _{t \rightarrow 0} \int_{\Omega_{+}} B^{\prime}(q) \frac{q_{t}-q}{t} d X=\int_{\Omega_{+}} B^{\prime}(q)\left(\omega_{\Gamma, V}-\frac{\zeta_{\Gamma, V}}{2}\right) d X,
$$

where $\omega_{\Gamma, V}$ and $\zeta_{\Gamma, V}$ are given in (4.37) and 4.27), respectively. This, together with (5.4) and (5.5), and our definition of $q$ and $q_{t}$, implies that

$$
\lim _{t \rightarrow 0} \delta_{2}(t)=\int_{\Omega_{+}}\left[(\nabla \cdot V) B\left(\psi_{\Gamma}-\frac{\phi_{\Gamma, \infty}}{2}\right)+B^{\prime}\left(\psi_{\Gamma}-\frac{\phi_{\Gamma, \infty}}{2}\right)\left(\omega_{\Gamma, V}-\frac{\zeta_{\Gamma, V}}{2}\right)\right] d X .
$$

Rearranging the terms, we have

$$
\begin{aligned}
\delta_{3}(t)= & \int_{\Omega_{+}} \frac{A_{V}(t)-I-t A_{V}^{\prime}(0)}{t} \nabla\left(\hat{\phi}_{\Gamma_{t}, \infty} \circ T_{t}\right) \cdot \nabla\left(\hat{\phi}_{0} \circ T_{t}\right) d X \\
& +\int_{\Omega_{+}} \frac{\nabla\left(\hat{\phi}_{\Gamma_{t}, \infty} \circ T_{t}\right)-\nabla \hat{\phi}_{\Gamma, \infty}}{t} \cdot \nabla\left(\hat{\phi}_{0} \circ T_{t}\right) d x \\
& +\int_{\Omega_{+}} \nabla \hat{\phi}_{\Gamma, \infty} \cdot \frac{\nabla\left(\hat{\phi}_{0} \circ T_{t}\right)-\nabla \hat{\phi}_{0}}{t} d X \\
& +\int_{\Omega_{+}} A_{V}^{\prime}(0) \nabla\left(\hat{\phi}_{\Gamma_{t}, \infty} \circ T_{t}\right) \cdot \nabla\left(\hat{\phi}_{0} \circ T_{t}\right) d X .
\end{aligned}
$$

Therefore, we have by 4.7 , 4.8), Lemma 4.2 (with $\hat{\phi}=\hat{\phi}_{\mathrm{C}}$ ), and Lemma 4.4 that

$$
\lim _{t \rightarrow 0} \delta_{3}(t)=\int_{\Omega_{+}}\left[\nabla \xi_{\Gamma, V} \cdot \nabla \hat{\phi}_{0}+\nabla \hat{\phi}_{\Gamma, \infty} \cdot \nabla\left(\nabla \hat{\phi}_{0} \cdot V\right)+A_{V}^{\prime}(0) \nabla \hat{\phi}_{\Gamma, \infty} \cdot \nabla \hat{\phi}_{0}\right] d X .
$$


It now follows from Definition 3.1, (5.2), (5.3), (5.6), and (5.7) that the first variation $\delta_{\Gamma} E[\Gamma]$ exists and is given by

$$
\begin{aligned}
\delta_{\Gamma, V} E[\Gamma]= & -\int_{\Omega} \frac{\varepsilon_{\Gamma}}{2} A_{V}^{\prime}(0) \nabla \psi_{\mathrm{r}} \cdot \nabla \psi_{\mathrm{r}} d X+\int_{\Omega} \varepsilon_{\Gamma} \nabla \psi_{\mathrm{r}} \cdot \nabla \xi_{\Gamma, V} d X-\int_{\Omega} \varepsilon_{\Gamma} \nabla \psi_{\mathrm{r}} \cdot \nabla \omega_{\Gamma, V} d X \\
& -\int_{\Omega_{+}}\left[(\nabla \cdot V) B\left(\psi_{\Gamma}-\frac{\phi_{\Gamma, \infty}}{2}\right)+B^{\prime}\left(\psi_{\Gamma}-\frac{\phi_{\Gamma, \infty}}{2}\right)\left(\omega_{\Gamma, V}-\frac{\zeta_{\Gamma, V}}{2}\right)\right] d X \\
& +\frac{\varepsilon_{-}-\varepsilon_{+}}{2} \int_{\Omega_{+}} \nabla \xi_{\Gamma, V} \cdot \nabla \hat{\phi}_{0} d X \\
& +\frac{\varepsilon_{-}-\varepsilon_{+}}{2} \int_{\Omega_{+}}\left[\nabla \hat{\phi}_{\Gamma, \infty} \cdot \nabla\left(\nabla \hat{\phi}_{0} \cdot V\right)+A_{V}^{\prime}(0) \nabla \hat{\phi}_{\Gamma, \infty} \cdot \nabla \hat{\phi}_{0}\right] d X \\
= & M_{1}+M_{2}+M_{3}+M_{4}+M_{5}+M_{6} .
\end{aligned}
$$

Step 2. We now simplify this expression. By Lemma 4.4 and our notation $\psi_{\mathrm{r}}=\psi_{\Gamma}-\hat{\phi}_{\Gamma, \infty}$, we can express the sum of the first two integrals above as

$$
\begin{aligned}
M_{1}+M_{2}= & -\int_{\Omega} \frac{\varepsilon_{\Gamma}}{2} A_{V}^{\prime}(0) \nabla\left(\psi_{\Gamma}-\hat{\phi}_{\Gamma, \infty}\right) \cdot \nabla\left(\psi_{\Gamma}-\hat{\phi}_{\Gamma, \infty}\right) d X \\
& -\int_{\Omega} \varepsilon_{\Gamma} A_{V}^{\prime}(0) \nabla \hat{\phi}_{\Gamma, \infty} \cdot \nabla\left(\psi_{\Gamma}-\hat{\phi}_{\Gamma, \infty}\right) d X \\
= & -\int_{\Omega} \frac{\varepsilon_{\Gamma}}{2} A_{V}^{\prime}(0) \nabla \psi_{\Gamma} \cdot \nabla \psi_{\Gamma} d X+\int_{\Omega} \frac{\varepsilon_{\Gamma}}{2} A_{V}^{\prime}(0) \nabla \hat{\phi}_{\Gamma, \infty} \cdot \nabla \hat{\phi}_{\Gamma, \infty} d X .
\end{aligned}
$$

Note that the last two integrals exist as the singularities $x_{i}(1 \leq i \leq N)$ of $\psi_{\Gamma}$ and $\hat{\phi}_{\Gamma, \infty}$ are outside the support of $V$ and $A_{V}^{\prime}(0)$ is given in (4.6). By (2.20) in Definition 2.1 and (2.11) we have

$$
\int_{\Omega}\left[\varepsilon_{\Gamma} \nabla \psi_{r} \cdot \nabla \eta+\chi_{+} B^{\prime}\left(\psi_{\Gamma}-\frac{\phi_{\Gamma, \infty}}{2}\right) \eta\right] d X=0
$$

for all $\eta \in C_{\mathrm{c}}^{1}(\Omega)$ and hence all $\eta \in H_{0}^{1}(\Omega)$. Setting $\eta=\omega_{\Gamma, V}$, we get the two- $\omega_{\Gamma, V}$ terms in (5.8) (one is $M_{3}$ and the other is part of $M_{4}$ ) cancelled:

$$
\int_{\Omega}\left[\varepsilon_{\Gamma} \nabla \psi_{\mathrm{r}} \cdot \nabla \omega_{\Gamma, V}+\chi_{+} B^{\prime}\left(\psi_{\Gamma}-\frac{\phi_{\Gamma, \infty}}{2}\right) \omega_{\Gamma, V}\right] d X=0 .
$$

To simplify $M_{5}$, we note that we can replace $\eta$ in 2.3 (with $\hat{\phi}=\hat{\phi}_{0}$ ) and 2.11) by $\xi_{\Gamma, V} \in H_{0}^{1}(\Omega)$, as $\left.\xi_{\Gamma, V}\right|_{\Omega_{-}} \in C^{2}\left(\Omega_{-}\right)$; cf. the remark below $(2.4)$ and that below $(2.12)$. It then follows that

$$
\begin{aligned}
M_{5} & =\frac{\varepsilon_{-}}{2} \int_{\Omega_{+}} \nabla \xi_{\Gamma, V} \cdot \nabla \hat{\phi}_{0} d X-\frac{\varepsilon_{+}}{2} \int_{\Omega_{+}} \nabla \xi_{\Gamma, V} \cdot \nabla \hat{\phi}_{0} d X \\
& =-\int_{\Omega} \frac{\varepsilon_{\Gamma}}{2} \nabla \xi_{\Gamma, V} \cdot \nabla \hat{\phi}_{0} d X+\frac{1}{2} \sum_{i=1}^{N} Q_{i} \xi_{\Gamma, V}\left(x_{i}\right) \quad\left[\text { by } 2.3 \text { with } \hat{\phi}=\hat{\phi}_{0}\right]
\end{aligned}
$$




$$
\begin{aligned}
& =-\int_{\Omega} \frac{\varepsilon_{\Gamma}}{2} \nabla \xi_{\Gamma, V} \cdot \nabla \hat{\phi}_{0} d X+\int_{\Omega} \frac{\varepsilon_{\Gamma}}{2} \nabla \hat{\phi}_{\Gamma, \infty} \cdot \nabla \xi_{\Gamma, V} d X \quad[\text { by 2.11] }] \\
& \left.=\int_{\Omega} \frac{\varepsilon_{\Gamma}}{2} \nabla \xi_{\Gamma, V} \cdot \nabla\left(\hat{\phi}_{\Gamma, \infty}-\hat{\phi}_{0}-\phi_{\Gamma, \infty}\right) d X \quad[\text { by } 2.5]\right] \\
& =-\int_{\Omega} \frac{\varepsilon_{\Gamma}}{2} A_{V}^{\prime}(0) \nabla \hat{\phi}_{\Gamma, \infty} \cdot \nabla\left(\hat{\phi}_{\Gamma, \infty}-\hat{\phi}_{0}-\phi_{\Gamma, \infty}\right) d X . \quad \text { [by Lemma 4.4 }
\end{aligned}
$$

Since $\hat{\phi}_{0}$ is harmonic in the support of $V$ that excludes all $x_{i}(i=1, \ldots, N)$, we have by the same calculations as in 4.32 that

$$
\nabla \cdot\left[\nabla\left(\nabla \hat{\phi}_{0} \cdot V\right)+A_{V}^{\prime}(0) \nabla \hat{\phi}_{0}\right]=0 \quad \text { in } \Omega .
$$

Thus, since the normal $n$ along $\Gamma$ points from $\Omega_{-}$to $\Omega_{+}$, we have by the Divergence Theorem that

$$
\begin{aligned}
& \frac{\varepsilon_{-}}{2} \int_{\Omega_{+}} \nabla \hat{\phi}_{\Gamma, \infty} \cdot\left[\nabla\left(\nabla \hat{\phi}_{0} \cdot V\right)+A_{V}^{\prime}(0) \nabla \hat{\phi}_{0}\right] d X \\
& =-\frac{\varepsilon_{-}}{2} \int_{\Gamma} \hat{\phi}_{\Gamma, \infty}\left[\nabla\left(\nabla \hat{\phi}_{0} \cdot V\right)+A_{V}^{\prime}(0) \nabla \hat{\phi}_{0}\right] \cdot n d S \\
& =-\frac{\varepsilon_{-}}{2} \int_{\Omega_{-}} \nabla \hat{\phi}_{\Gamma, \infty} \cdot\left[\nabla\left(\nabla \hat{\phi}_{0} \cdot V\right)+A_{V}^{\prime}(0) \nabla \hat{\phi}_{0}\right] d X
\end{aligned}
$$

Therefore, since $A_{V}^{\prime}(0)$ (cf. (4.6)) is symmetric,

$$
\begin{aligned}
M_{6} & =\frac{\varepsilon_{-}-\varepsilon_{+}}{2} \int_{\Omega_{+}} \nabla \hat{\phi}_{\Gamma, \infty} \cdot\left[\nabla\left(\nabla \hat{\phi}_{0} \cdot V\right)+A_{V}^{\prime}(0) \nabla \hat{\phi}_{0}\right] d X \\
& =-\int_{\Omega} \frac{\varepsilon_{\Gamma}}{2}\left[\nabla \hat{\phi}_{\Gamma, \infty} \cdot \nabla\left(\nabla \hat{\phi}_{0} \cdot V\right)+A_{V}^{\prime}(0) \nabla \hat{\phi}_{\Gamma, \infty} \cdot \nabla \hat{\phi}_{0}\right] d X \\
& =-\int_{\Omega} \frac{\varepsilon_{\Gamma}}{2} A_{V}^{\prime}(0) \nabla \hat{\phi}_{\Gamma, \infty} \cdot \nabla \hat{\phi}_{0} d X . \quad[\text { by 2.11) }]
\end{aligned}
$$

It now follows from $(5.8)-(5.12)$ that

$$
\begin{aligned}
\delta_{\Gamma, V} E[\Gamma]= & -\int_{\Omega} \frac{\varepsilon_{\Gamma}}{2} A_{V}^{\prime}(0) \nabla \psi_{\Gamma} \cdot \nabla \psi_{\Gamma} d X+\int_{\Omega} \frac{\varepsilon_{\Gamma}}{2} A_{V}^{\prime}(0) \nabla \hat{\phi}_{\Gamma, \infty} \cdot \nabla \phi_{\Gamma, \infty} d X \\
& +\int_{\Omega_{+}}\left[\frac{\zeta_{\Gamma, V}}{2} B^{\prime}\left(\psi_{\Gamma}-\frac{\phi_{\Gamma, \infty}}{2}\right)-(\nabla \cdot V) B\left(\psi_{\Gamma}-\frac{\phi_{\Gamma, \infty}}{2}\right)\right] d X \\
= & P_{1}+P_{2}+P_{3} .
\end{aligned}
$$

Step 3. We convert most of these volume integrals into surface integrals on $\Gamma$. We shall use the following identities that can be verified by using the Divergence Theorem and approximations by smooth functions:

$$
\int_{D}(\nabla \cdot U) \nabla a \cdot \nabla b d x=-\int_{D} U \cdot\left(\nabla^{2} a \nabla b+\nabla^{2} b \nabla a\right) d x+\int_{\partial D}(\nabla a \cdot \nabla b)(U \cdot \nu) d x,
$$




$$
\int_{D}(\nabla U) \nabla a \cdot \nabla b d x=-\int_{D} U \cdot\left(\Delta a \nabla b+\nabla^{2} b \nabla a\right) d x+\int_{\partial D}(\nabla a \cdot \nu)(\nabla b \cdot U) d x .
$$

Here, $D \subset \mathbb{R}^{3}$ is a bounded open set with a $C^{1}$ boundary $\partial D, U \in H^{1}\left(D, \mathbb{R}^{3}\right), a, b \in H^{2}(D)$, $\nabla^{2} a$ is the Hessian matrix of $a$, and $\nu$ is the unit exterior normal at the boundary $\partial D$. If in addition $\Delta a=\Delta b=0$ in $D$, then we have by (5.14) and (5.15) that

$$
\begin{aligned}
\int_{D}\left(\nabla U+(\nabla U)^{T}-(\nabla \cdot U) I\right) \nabla a \cdot \nabla b d x \\
\quad=\int_{\partial D}[(\nabla a \cdot U) \cdot(\nabla b \cdot \nu)+(\nabla b \cdot U) \cdot(\nabla a \cdot \nu)-(\nabla a \cdot \nabla b) \cdot(U \cdot \nu)] d S .
\end{aligned}
$$

Note that $V=0$ in a neighborhood of all $x_{i}(1 \leq i \leq N)$ and $V=0$ on $\partial \Omega$ and that the unit normal vector $n$ on $\Gamma$ points from $\Omega_{-}$to $\Omega_{+}$. By Theorem 2.1, $\Delta \psi_{\Gamma}=0$ on $\Omega_{-} \cap \operatorname{supp}(V)$ and $\varepsilon_{+} \Delta \psi_{\Gamma}=B^{\prime}\left(\psi_{\Gamma}-\phi_{\Gamma, \infty} / 2\right)$ on $\Omega_{+}$. Therefore, we have by (4.6), (5.14), and (5.15) that

$$
\begin{aligned}
& P_{1}=\int_{\Omega} \frac{\varepsilon_{\Gamma}}{2}\left[\nabla V+(\nabla V)^{T}-(\nabla \cdot V) I\right] \nabla \psi_{\Gamma} \cdot \nabla \psi_{\Gamma} d X \\
& =\int_{\Omega_{-}} \varepsilon_{-}(\nabla V) \nabla \psi_{\Gamma} \cdot \nabla \psi_{\Gamma} d X+\int_{\Omega_{+}} \varepsilon_{+}(\nabla V) \nabla \psi_{\Gamma} \cdot \nabla \psi_{\Gamma} d X \\
& -\int_{\Omega_{-}} \frac{\varepsilon_{-}}{2}(\nabla \cdot V) \nabla \psi_{\Gamma} \cdot \nabla \psi_{\Gamma} d X-\int_{\Omega_{+}} \frac{\varepsilon_{+}}{2}(\nabla \cdot V) \nabla \psi_{\Gamma} \cdot \nabla \psi_{\Gamma} d X \\
& =-\int_{\Omega_{-}} \varepsilon_{-} V \cdot\left(\Delta \psi_{\Gamma} \nabla \psi_{\Gamma}+\nabla^{2} \psi_{\Gamma} \nabla \psi_{\Gamma}\right) d X+\int_{\Gamma} \varepsilon_{-}\left(\nabla \psi_{\Gamma}^{-} \cdot V\right)\left(\nabla \psi_{\Gamma}^{-} \cdot n\right) d S \\
& -\int_{\Omega_{+}} \varepsilon_{+} V \cdot\left(\Delta \psi_{\Gamma} \nabla \psi_{\Gamma}+\nabla^{2} \psi_{\Gamma} \nabla \psi_{\Gamma}\right) d X-\int_{\Gamma} \varepsilon_{+}\left(\nabla \psi_{\Gamma}^{+} \cdot V\right)\left(\psi_{\Gamma}^{+} \cdot n\right) d S \\
& +\int_{\Omega_{-}} \varepsilon_{-} V \cdot \nabla^{2} \psi_{\Gamma} \nabla \psi_{\Gamma} d X-\int_{\Gamma} \frac{\varepsilon_{-}}{2}\left|\nabla \psi_{\Gamma}^{-}\right|^{2}(V \cdot n) d S \\
& +\int_{\Omega_{+}} \varepsilon_{+} V \cdot \nabla^{2} \psi_{\Gamma} \nabla \psi_{\Gamma} d X+\int_{\Gamma} \frac{\varepsilon_{+}}{2}\left|\nabla \psi_{\Gamma}^{+}\right|^{2}(V \cdot n) d S \\
& =-\int_{\Omega_{-}} \varepsilon_{-} \Delta \psi_{\Gamma}\left(\nabla \psi_{\Gamma} \cdot V\right) d X+\int_{\Gamma} \varepsilon_{-}\left(\nabla \psi_{\Gamma}^{-} \cdot V\right)\left(\nabla \psi_{\Gamma}^{-} \cdot n\right) d S-\int_{\Gamma} \frac{\varepsilon_{-}}{2}\left|\nabla \psi_{\Gamma}^{-}\right|^{2}(V \cdot n) d S \\
& -\int_{\Omega_{+}} \varepsilon_{+} \Delta \psi_{\Gamma}\left(\nabla \psi_{\Gamma} \cdot V\right) d X-\int_{\Gamma} \varepsilon_{+}\left(\nabla \psi_{\Gamma}^{+} \cdot V\right)\left(\nabla \psi_{\Gamma}^{+} \cdot n\right) d S+\int_{\Gamma} \frac{\varepsilon_{+}}{2}\left|\nabla \psi_{\Gamma}^{+}\right|^{2}(V \cdot n) d S \\
& =\int_{\Gamma} \varepsilon_{-}\left(\nabla \psi_{\Gamma}^{-} \cdot V\right)\left(\nabla \psi_{\Gamma}^{-} \cdot n\right) d S-\int_{\Gamma} \varepsilon_{+}\left(\nabla \psi_{\Gamma}^{+} \cdot V\right)\left(\nabla \psi_{\Gamma}^{+} \cdot n\right) d S \\
& -\int_{\Gamma} \frac{\varepsilon_{-}}{2}\left|\nabla \psi_{\Gamma}^{-}\right|^{2}(V \cdot n) d S+\int_{\Gamma} \frac{\varepsilon_{+}}{2}\left|\nabla \psi_{\Gamma}^{+}\right|^{2}(V \cdot n) d S \\
& -\int_{\Omega_{+}} B^{\prime}\left(\psi_{\Gamma}-\frac{\phi_{\Gamma, \infty}}{2}\right)\left(\nabla \psi_{\Gamma} \cdot V\right) d X
\end{aligned}
$$


where a superscript - or + denotes the restriction from $\Omega_{-}$or $\Omega_{+}$, respectively.

Since $\hat{\phi}_{\Gamma, \infty}$ and $\phi_{\Gamma, \infty}$ are harmonic in $\Omega_{-} \cap \operatorname{supp}(V)$ and $\Omega_{+}$, and since the normal $n$ points from $\Omega_{-}$to $\Omega_{+}$, we have by (4.6), (5.16), and the notation of jumps (2.10) that

$$
\begin{aligned}
P_{2}= & \int_{\Omega_{-}} \frac{\varepsilon_{-}}{2}\left[(\nabla \cdot V) I-\nabla V-(\nabla V)^{T}\right] \nabla \hat{\phi}_{\Gamma, \infty} \cdot \nabla \phi_{\Gamma, \infty} d X \\
& +\int_{\Omega_{+}} \frac{\varepsilon_{+}}{2}\left[(\nabla \cdot V) I-\nabla V-(\nabla V)^{T}\right] \nabla \hat{\phi}_{\Gamma, \infty} \cdot \nabla \phi_{\Gamma, \infty} d X \\
= & \frac{1}{2} \int_{\Gamma} \llbracket \varepsilon_{\Gamma}\left(\nabla \hat{\phi}_{\Gamma, \infty} \cdot V\right)\left(\nabla \phi_{\Gamma, \infty} \cdot n\right)+\varepsilon_{\Gamma}\left(\nabla \hat{\phi}_{\Gamma, \infty} \cdot n\right)\left(\nabla \phi_{\Gamma, \infty} \cdot V\right) \\
& -\varepsilon_{\Gamma}\left(\nabla \hat{\phi}_{\Gamma, \infty} \cdot \nabla \phi_{\Gamma, \infty}\right)(V \cdot n) \rrbracket_{\Gamma} d S .
\end{aligned}
$$

Using the Divergence Theorem and noting again that the normal $n$ at $\Gamma$ points from $\Omega_{-}$ to $\Omega_{+}$, we obtain

$$
\begin{aligned}
P_{3}= & \int_{\Omega_{+}} \frac{\zeta_{\Gamma, V}}{2} B^{\prime}\left(\psi_{\Gamma}-\frac{\phi_{\Gamma, \infty}}{2}\right) d X+\int_{\Omega_{+}} V \cdot B^{\prime}\left(\psi_{\Gamma}-\frac{\phi_{\Gamma, \infty}}{2}\right)\left(\nabla \psi_{\Gamma}-\frac{\nabla \phi_{\Gamma, \infty}}{2}\right) d X \\
& +\int_{\Gamma} B\left(\psi_{\Gamma}-\frac{\phi_{\Gamma, \infty}}{2}\right)(V \cdot n) d S \\
= & \int_{\Omega_{+}}\left[\frac{1}{2}\left(\zeta_{\Gamma, V}-\nabla \phi_{\Gamma, \infty} \cdot V\right)+\nabla \psi_{\Gamma} \cdot V\right] B^{\prime}\left(\psi_{\Gamma}-\frac{\phi_{\Gamma, \infty}}{2}\right) d X \\
& +\int_{\Gamma} B\left(\psi_{\Gamma}-\frac{\phi_{\Gamma, \infty}}{2}\right)(V \cdot n) d S .
\end{aligned}
$$

It now follows from $(5.13)$ and $(5.17)-(5.19)$ that

$$
\begin{aligned}
\delta_{\Gamma, V} E[\Gamma]= & \int_{\Gamma} \varepsilon_{-}\left(\nabla \psi_{\Gamma}^{-} \cdot V\right)\left(\nabla \psi_{\Gamma}^{-} \cdot n\right) d S-\int_{\Gamma} \varepsilon_{+}\left(\nabla \psi_{\Gamma}^{+} \cdot V\right)\left(\nabla \psi_{\Gamma}^{+} \cdot n\right) d S \\
& -\int_{\Gamma} \frac{\varepsilon_{-}}{2}\left|\nabla \psi_{\Gamma}^{-}\right|^{2}(V \cdot n) d S+\int_{\Gamma} \frac{\varepsilon_{+}}{2}\left|\nabla \psi_{\Gamma}^{+}\right|^{2}(V \cdot n) d S \\
& +\frac{1}{2} \int_{\Gamma} \llbracket \varepsilon_{\Gamma}\left(\nabla \hat{\phi}_{\Gamma, \infty} \cdot V\right)\left(\nabla \phi_{\Gamma, \infty} \cdot n\right) \rrbracket_{\Gamma} d S \\
& +\frac{1}{2} \int_{\Gamma} \llbracket \varepsilon_{\Gamma}\left(\nabla \hat{\phi}_{\Gamma, \infty} \cdot n\right)\left(\nabla \phi_{\Gamma, \infty} \cdot V\right) \rrbracket_{\Gamma} d S \\
& -\frac{1}{2} \int_{\Gamma} \llbracket \varepsilon_{\Gamma}\left(\nabla \hat{\phi}_{\Gamma, \infty} \cdot \nabla \phi_{\Gamma, \infty}\right)(V \cdot n) \rrbracket_{\Gamma} d S \\
& +\int_{\Omega_{+}} \frac{1}{2}\left(\zeta_{\Gamma, V}-\nabla \phi_{\Gamma, \infty} \cdot V\right) B^{\prime}\left(\psi_{\Gamma}-\frac{\phi_{\Gamma, \infty}}{2}\right) d X \\
& +\int_{\Gamma} B\left(\psi_{\Gamma}-\frac{\phi_{\Gamma, \infty}}{2}\right)(V \cdot n) d S .
\end{aligned}
$$


Step 4. We express the surface integrals into those with the factor $V \cdot n$ in the integrand. Note that on each side of $\Gamma$, we can write

$$
\nabla \psi_{\Gamma}=\left(\nabla \psi_{\Gamma} \cdot n\right) n+\nabla_{\Gamma} \psi_{\Gamma}=\partial_{n} \psi_{\Gamma} n+\nabla_{\Gamma} \psi_{\Gamma} \quad \text { on } \Gamma
$$

where $\nabla_{\Gamma} \psi_{\Gamma}=(I-n \otimes n) \nabla \psi_{\Gamma}$ is the tangential derivative. Clearly $n \cdot \nabla_{\Gamma} \psi_{\Gamma}=0$. Moreover, $\nabla_{\Gamma} \psi_{\Gamma}^{+}=\nabla_{\Gamma} \psi_{\Gamma}^{-}$on $\Gamma$. Thus,

$$
\nabla \psi_{\Gamma}^{+}-\nabla \psi_{\Gamma}^{-}=\left(\partial_{n} \psi_{\Gamma}^{+}-\partial_{n} \psi_{\Gamma}^{-}\right) n \quad \text { on } \Gamma .
$$

By Theorem 2.1, we have also $\varepsilon_{+} \nabla \psi_{\Gamma}^{+} \cdot n=\varepsilon_{-} \nabla \psi_{\Gamma}^{-} \cdot n=\varepsilon_{\Gamma} \nabla \psi_{\Gamma} \cdot n$ on $\Gamma$. Therefore, the first four terms in (5.20) are

$$
\begin{aligned}
\int_{\Gamma} \varepsilon_{-} & \left(\nabla \psi_{\Gamma}^{-} \cdot V\right)\left(\nabla \psi_{\Gamma}^{-} \cdot n\right) d S-\int_{\Gamma} \varepsilon_{+}\left(\nabla \psi_{\Gamma}^{+} \cdot V\right)\left(\nabla \psi_{\Gamma}^{+} \cdot n\right) d S \\
- & \int_{\Gamma} \frac{\varepsilon_{-}}{2}\left|\nabla \psi_{\Gamma}^{-}\right|^{2}(V \cdot n) d S+\int_{\Gamma} \frac{\varepsilon_{+}}{2}\left|\nabla \psi_{\Gamma}^{+}\right|^{2}(V \cdot n) d S \\
= & -\int_{\Gamma} \varepsilon_{\Gamma} \partial_{n} \psi_{\Gamma}\left(\partial_{n} \psi_{\Gamma}^{+}-\partial_{n} \psi_{\Gamma}^{-}\right)(V \cdot n) d S \\
& +\int_{\Gamma} \frac{\varepsilon_{+}}{2}\left|\partial_{n} \psi_{\Gamma}^{+}\right|^{2}(V \cdot n) d S+\int_{\Gamma} \frac{\varepsilon_{+}}{2}\left|\nabla_{\Gamma} \psi_{\Gamma}\right|^{2}(V \cdot n) d S \\
& -\int_{\Gamma} \frac{\varepsilon_{-}}{2}\left|\partial_{n} \psi_{\Gamma}^{-}\right|^{2}(V \cdot n) d S-\int_{\Gamma} \frac{\varepsilon_{-}}{2}\left|\nabla_{\Gamma} \psi_{\Gamma}\right|^{2}(V \cdot n) d S \\
= & -\int_{\Gamma} \varepsilon_{+}\left|\partial_{n} \psi_{\Gamma}^{+}\right|^{2}(V \cdot n) d S+\int_{\Gamma} \varepsilon_{-}\left|\partial_{n} \psi_{\Gamma}^{-}\right|^{2}(V \cdot n) d S \\
& +\int_{\Gamma} \frac{\varepsilon_{+}}{2}\left|\partial_{n} \psi_{\Gamma}^{+}\right|^{2}(V \cdot n) d S+\int_{\Gamma} \frac{\varepsilon_{+}}{2}\left|\nabla_{\Gamma} \psi_{\Gamma}\right|^{2}(V \cdot n) d S \\
& -\int_{\Gamma} \frac{\varepsilon_{-}}{2}\left|\partial_{n} \psi_{\Gamma}^{-}\right|^{2}(V \cdot n) d S-\int_{\Gamma} \frac{\varepsilon_{-}}{2}\left|\nabla_{\Gamma} \psi_{\Gamma}\right|^{2}(V \cdot n) d S \\
= & -\frac{1}{2}\left(\frac{1}{\varepsilon_{+}}-\frac{1}{\varepsilon_{-}}\right) \int_{\Gamma}\left|\varepsilon_{\Gamma} \partial_{n} \psi_{\Gamma}\right|^{2}(V \cdot n) d S+\frac{\varepsilon_{+}-\varepsilon_{-}}{2} \int_{\Gamma}\left|\nabla_{\Gamma} \psi_{\Gamma}\right|^{2}(V \cdot n) d S .
\end{aligned}
$$

Similarly, on each side of $\Gamma$, we have with $u_{\Gamma}=\phi_{\Gamma, \infty}$ or $\hat{\phi}_{\Gamma, \infty}$ that

$$
\begin{aligned}
\nabla u_{\Gamma} \cdot V & =\left(\partial_{n} u_{\Gamma} n+\nabla_{\Gamma} u_{\Gamma}\right) \cdot((V \cdot n) n+(I-n \otimes n) V) \\
& =\partial_{n} u_{\Gamma}(V \cdot n)+\nabla_{\Gamma} u_{\Gamma}(I-n \otimes n) V .
\end{aligned}
$$

Moreover, $\varepsilon_{+} \partial_{n} u_{\Gamma}^{+}=\varepsilon_{-} \partial_{n} u_{\Gamma}^{-}$and $\partial_{\Gamma} u_{\Gamma}^{+}=\partial_{\Gamma} u_{\Gamma}^{-}$on $\Gamma$. Therefore, the next three terms in (5.20) become

$$
\begin{aligned}
& \frac{1}{2} \int_{\Gamma} \llbracket \varepsilon_{\Gamma}\left(\nabla \hat{\phi}_{\Gamma, \infty} \cdot V\right)\left(\nabla \phi_{\Gamma, \infty} \cdot n\right) \rrbracket_{\Gamma} d S+\frac{1}{2} \int_{\Gamma} \llbracket \varepsilon_{\Gamma}\left(\nabla \hat{\phi}_{\Gamma, \infty} \cdot n\right)\left(\nabla \phi_{\Gamma, \infty} \cdot V\right) \rrbracket_{\Gamma} d S \\
& \quad-\frac{1}{2} \int_{\Gamma} \llbracket \varepsilon_{\Gamma}\left(\nabla \hat{\phi}_{\Gamma, \infty} \cdot \nabla \phi_{\Gamma, \infty}\right)(V \cdot n) \rrbracket_{\Gamma} d S
\end{aligned}
$$




$$
\begin{aligned}
= & \int_{\Gamma} \llbracket \varepsilon_{\Gamma} \partial_{n} \hat{\phi}_{\Gamma, \infty} \partial_{n} \phi_{\Gamma, \infty} \rrbracket_{\Gamma}(V \cdot n) d S \\
& -\frac{1}{2} \int_{\Gamma} \llbracket \varepsilon_{\Gamma}\left(\partial_{n} \hat{\phi}_{\Gamma, \infty} \partial_{n} \phi_{\Gamma, \infty}+\nabla_{\Gamma} \hat{\phi}_{\Gamma, \infty} \cdot \nabla_{\Gamma} \phi_{\Gamma, \infty}\right) \rrbracket_{\Gamma}(V \cdot n) d S \\
= & \frac{1}{2} \int_{\Gamma} \llbracket \varepsilon_{\Gamma} \partial_{n} \hat{\phi}_{\Gamma, \infty} \partial_{n} \phi_{\Gamma, \infty} \rrbracket_{\Gamma}(V \cdot n) d S-\frac{1}{2} \int_{\Gamma} \llbracket \varepsilon_{\Gamma} \nabla_{\Gamma} \hat{\phi}_{\Gamma, \infty} \cdot \nabla_{\Gamma} \phi_{\Gamma, \infty} \rrbracket_{\Gamma}(V \cdot n) d S .
\end{aligned}
$$

It now follows from $5.20-5.22$ that

$$
\begin{aligned}
& \delta_{\Gamma, V} E[\Gamma]=-\frac{1}{2}\left(\frac{1}{\varepsilon_{+}}-\frac{1}{\varepsilon_{-}}\right) \int_{\Gamma}\left|\varepsilon_{\Gamma} \partial_{n} \psi_{\Gamma}\right|^{2}(V \cdot n) d S+\frac{\varepsilon_{+}-\varepsilon_{-}}{2} \int_{\Gamma}\left|\nabla_{\Gamma} \psi_{\Gamma}\right|^{2}(V \cdot n) d S \\
& +\frac{1}{2} \int_{\Gamma} \llbracket \varepsilon_{\Gamma} \partial_{n} \hat{\phi}_{\Gamma, \infty} \partial_{n} \phi_{\Gamma, \infty} \rrbracket_{\Gamma}(V \cdot n) d S-\frac{1}{2} \int_{\Gamma} \llbracket \varepsilon_{\Gamma} \nabla_{\Gamma} \hat{\phi}_{\Gamma, \infty} \cdot \nabla_{\Gamma} \phi_{\Gamma, \infty} \rrbracket_{\Gamma}(V \cdot n) d S \\
& +\int_{\Omega_{+}} \frac{1}{2}\left(\zeta_{\Gamma, V}-\nabla \phi_{\Gamma, \infty} \cdot V\right) B^{\prime}\left(\psi_{\Gamma}-\frac{\phi_{\Gamma, \infty}}{2}\right) d X \\
& +\int_{\Gamma} B\left(\psi_{\Gamma}-\frac{\phi_{\Gamma, \infty}}{2}\right)(V \cdot n) d S
\end{aligned}
$$

Step 5. We finally rewrite the volume integral above into a surface integral on the boundary $\Gamma$. Recall from the beginning of Subsection 3.2 that the signed distance function $\phi: \mathbb{R}^{3} \rightarrow \mathbb{R}$ with respect to $\Gamma$ is a $C^{3}$-function and $\nabla \phi \neq 0$ in the neighborhood $\mathcal{N}_{0}(\Gamma)$ of $\Gamma$. We extend $n=\nabla \phi$ on $\Gamma$ to $\mathcal{N}_{0}(\Gamma)$, i.e., we define $n=\nabla \phi$ at every point in $\mathcal{N}_{0}(\Gamma)$. Note that $n \in C^{2}\left(\mathcal{N}_{0}(\Gamma)\right)$. Since $V \in \mathcal{V}$ vanishes outside $\mathcal{N}_{0}(\Gamma)$, both the normal component $(V \cdot n) n$ and the tangential component $V-(V \cdot n) n=(I-n \otimes n) V$ of $V$ are in the class of vector fields $\mathcal{V}$; cf. (3.6). Since

$$
V=(V \cdot n) n+(I-n \otimes n) V \quad \text { and } \quad(I-n \otimes n) V \cdot n=0,
$$

we have by Lemma 4.3 that

$$
\begin{aligned}
\zeta_{\Gamma, V}-\nabla \phi_{\Gamma, \infty} \cdot V & =\zeta_{\Gamma,(V \cdot n) n+(I-n \otimes n) V}-\nabla \phi_{\Gamma, \infty} \cdot[(V \cdot n) n+(I-n \otimes n) V] \\
& =\zeta_{\Gamma,(V \cdot n) n}-\nabla \phi_{\Gamma, \infty} \cdot(V \cdot n) n+\zeta_{\Gamma,(I-n \otimes n) V}-\nabla \phi_{\Gamma, \infty} \cdot(I-n \otimes n) V \\
& =\zeta_{\Gamma,(V \cdot n) n}-\nabla \phi_{\Gamma, \infty} \cdot(V \cdot n) n \quad \text { in } \Omega .
\end{aligned}
$$

Therefore, we may assume that

$$
V=(V \cdot n) n \quad \text { in } \mathcal{N}_{0}(\Gamma) .
$$

By Lemma 4.3, $\zeta_{\Gamma, V}^{\mathrm{s}} \in H^{2}\left(\Omega_{\mathrm{s}}\right)$ for $\mathrm{s}=-$ or + . Thus, by 4.28$), \Delta\left(\nabla \phi_{\Gamma, \infty} \cdot V\right) \in L^{2}\left(\Omega_{\mathrm{s}}\right)$ for $\mathrm{s}=-$ or + . Therefore,

$$
\nabla \phi_{\Gamma, \infty} \cdot V \in H^{2}\left(\Omega_{\mathrm{s}}\right) \quad \text { for } \mathrm{s}=- \text { or }+.
$$

Recall from (5.1) that $\psi_{\mathrm{r}}=\psi_{\Gamma}-\hat{\phi}_{\Gamma, \infty} \in H_{0}^{1}(\Omega)$. Note by Theorem 2.1 that $\Delta \psi_{\mathrm{r}}=0$ in $\Omega_{-}$and $\varepsilon_{+} \Delta \psi_{\mathrm{r}}=B^{\prime}\left(\psi_{\Gamma}-\phi_{\Gamma, \infty} / 2\right)$ in $\Omega_{+}$. Note also by 4.28$)$ in Lemma 4.3 that $\Delta\left(\zeta_{\Gamma, V}-\nabla \phi_{\Gamma, \infty} \cdot V\right)=$ 
0 in $\Omega_{-} \cup \Omega_{+}$. We then obtain by Green's second identity with our convention that the normal $n$ at $\Gamma$ pointing from $\Omega_{-}$to $\Omega_{+}$and the fact that $\llbracket \varepsilon_{\Gamma} \zeta_{\Gamma, V} \partial_{n} \psi_{\mathrm{r}} \rrbracket_{\Gamma}=0$ which follows from the third equation in 2.22 that twice of the volume term in 5.23 is

$$
\begin{aligned}
Q:= & \int_{\Omega_{+}}\left(\zeta_{\Gamma, V}-\nabla \phi_{\Gamma, \infty} \cdot V\right) B^{\prime}\left(\psi_{\Gamma}-\frac{\phi_{\Gamma, \infty}}{2}\right) d X \\
= & \int_{\Omega_{+}} \varepsilon_{+}\left[\left(\zeta_{\Gamma, V}-\nabla \phi_{\Gamma, \infty} \cdot V\right) \Delta \psi_{\mathrm{r}}-\psi_{\mathrm{r}} \Delta\left(\zeta_{\Gamma, V}-\nabla \phi_{\Gamma, \infty} \cdot V\right)\right] d X \\
& +\int_{\Omega_{-}} \varepsilon_{-}\left[\left(\zeta_{\Gamma, V}-\nabla \phi_{\Gamma, \infty} \cdot V\right) \Delta \psi_{\mathrm{r}}-\psi_{\mathrm{r}} \Delta\left(\zeta_{\Gamma, V}-\nabla \phi_{\Gamma, \infty} \cdot V\right)\right] d X \\
= & -\int_{\Gamma} \llbracket \varepsilon_{\Gamma}\left[\left(\zeta_{\Gamma, V}-\nabla \phi_{\Gamma, \infty} \cdot V\right) \partial_{n} \psi_{\mathrm{r}}-\psi_{\mathrm{r}} \partial_{n}\left(\zeta_{\Gamma, V}-\nabla \phi_{\Gamma, \infty} \cdot V\right)\right] \rrbracket_{\Gamma} d S \\
= & \int_{\Gamma} \llbracket \varepsilon_{\Gamma}\left(\nabla \phi_{\Gamma, \infty} \cdot V\right) \partial_{n} \psi_{\mathrm{r}} \rrbracket_{\Gamma} d S+\int_{\Gamma} \llbracket \varepsilon_{\Gamma} \psi_{\mathrm{r}} \partial_{n} \zeta_{\Gamma, V} \rrbracket_{\Gamma} d S-\int_{\Gamma} \llbracket \varepsilon_{\Gamma} \psi_{\mathrm{r}} \partial_{n}\left(\nabla \phi_{\Gamma, \infty} \cdot V\right) \rrbracket_{\Gamma} d S \\
= & Q_{1}+Q_{2}-Q_{3} .
\end{aligned}
$$

It follows from (5.24) that

$$
Q_{1}=\int_{\Gamma} \llbracket \varepsilon_{\Gamma}\left(\nabla \phi_{\Gamma, \infty} \cdot V\right) \partial_{n} \psi_{\mathrm{r}} \rrbracket_{\Gamma} d S=\int_{\Gamma} \llbracket \varepsilon_{\Gamma} \partial_{n} \phi_{\Gamma, \infty} \partial_{n} \psi_{\mathrm{r}} \rrbracket_{\Gamma}(V \cdot n) d S .
$$

Since $\llbracket \phi_{\mathrm{r}} \rrbracket_{\Gamma}=0$ and $\llbracket \varepsilon_{\Gamma} \partial_{n} \phi_{\Gamma, \infty} \rrbracket_{\Gamma}=0$, we have by Lemma 4.3 (cf. 4.29$)$ ) that

$$
\begin{aligned}
Q_{2} & =\int_{\Gamma} \llbracket \varepsilon_{\Gamma} \psi_{\mathrm{r}} \partial_{n} \zeta_{\Gamma, V} \rrbracket_{\Gamma} d S \\
& =-\int_{\Gamma} \llbracket \varepsilon_{\Gamma} \psi_{\mathrm{r}} A_{V}^{\prime}(0) \nabla \phi_{\Gamma, \infty} \cdot n \rrbracket_{\Gamma} d S \\
& =\int_{\Gamma} \llbracket \varepsilon_{\Gamma} \psi_{\mathrm{r}}\left[\nabla V+(\nabla V)^{T}-(\nabla \cdot V) I\right] \nabla \phi_{\Gamma, \infty} \cdot n \rrbracket_{\Gamma} d S \\
& =\int_{\Gamma} \llbracket \varepsilon_{\Gamma} \psi_{\mathrm{r}}\left[\nabla V+(\nabla V)^{T}\right] \nabla \phi_{\Gamma, \infty} \cdot n \rrbracket_{\Gamma} d S \\
& =\int_{\Gamma} \llbracket \varepsilon_{\Gamma} \psi_{\mathrm{r}} \nabla \phi_{\Gamma, \infty} \cdot\left[\nabla V+(\nabla V)^{T}\right] n \rrbracket_{\Gamma} d S .
\end{aligned}
$$

Denoting by $n^{j}$ the $j$ th component of $n$ and noting that $\partial_{i} n^{j} n^{j}=(1 / 2) \partial_{i}\|n\|^{2}=0$, we obtain on each side of $\Gamma$ (i.e., on $\mathcal{N}_{0}(\Gamma) \cup \Omega_{-}$and $\mathcal{N}_{0}(\Gamma) \cup \Omega_{+}$) that

$$
\begin{aligned}
\nabla \phi_{\Gamma, \infty} & \cdot\left(\nabla V+(\nabla V)^{T}\right) n \\
= & \partial_{i} \phi_{\Gamma, \infty}\left(\partial_{j} V^{i}+\partial_{i} V^{j}\right) n^{j} \\
= & \partial_{i} \phi_{\Gamma, \infty} \partial_{j}\left((V \cdot n) n^{i}\right) n^{j}+\partial_{i} \phi_{\Gamma, \infty} \partial_{i}\left((V \cdot n) n^{j}\right) n^{j} \quad \text { [by (5.24)] } \\
= & \partial_{i} \phi_{\Gamma, \infty} \partial_{j}(V \cdot n) n^{i} n^{j}+\partial_{i} \phi_{\Gamma, \infty}(V \cdot n) \partial_{j} n^{i} n^{j}
\end{aligned}
$$




$$
\begin{gathered}
\quad+\partial_{i} \phi_{\Gamma, \infty} \partial_{i}(V \cdot n) n^{j} n^{j}+\partial_{i} \phi_{\Gamma, \infty}(V \cdot n) \partial_{i} n^{j} n^{j} \\
=\left(\nabla \phi_{\Gamma, \infty} \cdot n\right) \nabla(V \cdot n) \cdot n+\nabla \phi_{\Gamma, \infty} \cdot((\nabla n) n)(V \cdot n)+\nabla \phi_{\Gamma, \infty} \cdot \nabla(V \cdot n) .
\end{gathered}
$$

This and (5.28), together with the fact that $\llbracket \varepsilon_{\Gamma} \nabla \phi_{\Gamma, \infty} \cdot n \rrbracket_{\Gamma}=0$ on $\Gamma$, lead to

$$
\begin{aligned}
Q_{2} & =\int_{\Gamma} \llbracket \varepsilon_{\Gamma} \psi_{\mathrm{r}} \nabla \phi_{\Gamma, \infty} \cdot(\nabla n) n \rrbracket_{\Gamma}(V \cdot n) d S+\int_{\Gamma} \llbracket \varepsilon_{\Gamma} \psi_{\mathrm{r}} \nabla \phi_{\Gamma, \infty} \cdot \nabla(V \cdot n) \rrbracket_{\Gamma} d S \\
& =Q_{2,1}+Q_{2,2} .
\end{aligned}
$$

To further simplify these terms, let us recall the surface divergence $\nabla_{\Gamma} v$ for a vector field $v$ along the boundary $\Gamma$ and its integral on $\Gamma$

$$
\begin{aligned}
& \nabla_{\Gamma} \cdot v=\nabla \cdot v-(\nabla v) n \cdot n, \\
& \int_{\Gamma} \nabla_{\Gamma} \cdot v d S=2 \int_{\Gamma} H(v \cdot n) d S,
\end{aligned}
$$

where $\mathrm{H}$ is the mean curvature; cf. [18] (Section 5 of Chapter 9).

Consider the term $Q_{2,1}$ in $(5.29)$. Since $n=\nabla \phi$ is a unit vector field, we have $n \cdot(\nabla n) n=$ $n^{i} \partial_{j} n^{i} n^{j}=(1 / 2) n^{j} \partial_{j}\left(n^{i} n^{i}\right)=0$. Hence, on each side of $\Gamma$, we have

$$
\nabla \phi_{\Gamma, \infty} \cdot(\nabla n) n=\nabla_{\Gamma} \phi_{\Gamma, \infty} \cdot(\nabla n) n .
$$

Let us denote $\alpha_{\Gamma}=\psi_{\mathrm{r}} \nabla_{\Gamma} \phi_{\Gamma, \infty}$ and note that $\llbracket \alpha_{\Gamma} \rrbracket_{\Gamma}=0$. Hence $\alpha_{\Gamma} \in H^{1}\left(\mathcal{N}_{0}(\Gamma), \mathbb{R}^{3}\right)$. Note also that $\alpha_{\Gamma} \cdot n=0$. Thus,

$$
\begin{aligned}
\left(\nabla \alpha_{\Gamma}\right) n \cdot n+\alpha_{\Gamma} \cdot(\nabla n) n & =\left[\left(\nabla \alpha_{\Gamma}\right)^{T} n+(\nabla n)^{T} \alpha_{\Gamma}\right] \cdot n \\
& =\nabla\left(\alpha_{\Gamma} \cdot n\right) \cdot n \\
& =0 \quad \text { in } \mathcal{N}_{0}(\Gamma) .
\end{aligned}
$$

This implies that

$$
\left(\nabla \alpha_{\Gamma} n\right) \cdot n=-\alpha_{\Gamma} \cdot(\nabla n) n \in H^{1}\left(\mathcal{N}_{0}(\Gamma)\right) .
$$

By (5.24), we have for $\mathrm{s}=-$ or + that

$$
\begin{aligned}
\nabla\left(\nabla \phi_{\Gamma, \infty} \cdot V\right) \cdot n & =\nabla\left(\left(\nabla \phi_{\Gamma, \infty} \cdot n\right)(V \cdot n)\right) \cdot n \\
& =\left(\nabla\left(\nabla \phi_{\Gamma, \infty} \cdot n\right) \cdot n\right)(V \cdot n)+\left(\nabla \phi_{\Gamma, \infty} \cdot n\right) \nabla(V \cdot n) \cdot n \quad \text { in } \Omega_{\mathrm{s}} \cap \mathcal{N}_{0}(\Gamma) .
\end{aligned}
$$

This, together with (2.6) and (5.25), implies for $\mathrm{s}=-$ or + that

$$
\left(\nabla\left(\nabla \phi_{\Gamma, \infty} \cdot n\right) \cdot n\right)(V \cdot n) \in H^{1}\left(\Omega_{\mathrm{s}} \cap \mathcal{N}_{0}(\Gamma)\right) .
$$

Therefore, since $\nabla_{\Gamma} \phi_{\Gamma, \infty}=\nabla \phi_{\Gamma, \infty}-\left(\nabla \phi_{\Gamma, \infty} \cdot n\right) n, \Delta \phi_{\Gamma, \infty}=0$ in $\Omega_{-}$and $\Omega_{+}$, and $\psi_{\mathrm{r}}$ and $\phi_{\Gamma, \infty}$ are in $W^{1, \infty}$ on each side of $\Gamma$, we can verify that for $\mathrm{s}=-$ or +

$$
\left(\nabla \cdot \alpha_{\Gamma}\right)(V \cdot n)=\left(\nabla \psi_{\mathrm{r}} \cdot \nabla \phi_{\Gamma, \infty}\right)(V \cdot n)-\left(\nabla \psi_{\mathrm{r}} \cdot n\right)\left(\nabla \phi_{\Gamma, \infty} \cdot n\right)(V \cdot n)
$$




$$
-\psi_{\mathrm{r}}\left(\nabla\left(\nabla \phi_{\Gamma, \infty} \cdot n\right) \cdot n\right)(V \cdot n)-\psi_{\mathrm{r}}\left(\nabla \phi_{\Gamma, \infty} \cdot n\right)(\nabla \cdot n)(V \cdot n) \in H^{1}\left(\Omega_{\mathrm{s}} \cap \mathcal{N}_{0}(\Gamma)\right) .
$$

By (5.34), (5.36), and (5.30) (with $\alpha_{\Gamma}$ replacing $v$ ), we have for $\mathrm{s}=-$ or + that

$$
\left(\nabla_{\Gamma} \cdot \alpha_{\Gamma}\right)(V \cdot n)=\left(\nabla \cdot \alpha_{\Gamma}\right)(V \cdot n)-\left(\nabla \alpha_{\Gamma} n \cdot n\right)(V \cdot n) \in H^{1}\left(\Omega_{\mathrm{s}} \cap \mathcal{N}_{0}(\Gamma)\right) .
$$

With all the regularity results (5.34), (5.36), and (5.37), we have now by (5.32), (5.33), and (5.30) (with $\alpha_{\Gamma}$ replacing $v$ ) that

$$
\begin{aligned}
Q_{2,1} & =\int_{\Gamma} \llbracket \varepsilon_{\Gamma} \alpha_{\Gamma} \cdot(\nabla n) n \rrbracket_{\Gamma}(V \cdot n) d S \\
& =-\int_{\Gamma} \llbracket \varepsilon_{\Gamma}\left(\nabla \alpha_{\Gamma}\right) n \cdot n \rrbracket_{\Gamma}(V \cdot n) d S \\
& =\int_{\Gamma} \llbracket \varepsilon_{\Gamma}\left(\nabla_{\Gamma} \cdot \alpha_{\Gamma}-\nabla \cdot \alpha_{\Gamma}\right) \rrbracket_{\Gamma}(V \cdot n) d S .
\end{aligned}
$$

Consider now the term $Q_{2,2}$ in $(5.29)$. On each side of $\Gamma$,

$$
\begin{aligned}
\nabla \phi_{\Gamma, \infty} \cdot \nabla(V \cdot n) & =\left[\left(\nabla \phi_{\Gamma, \infty} \cdot n\right) n+\nabla_{\Gamma} \phi_{\Gamma, \infty}\right] \cdot\left[(\nabla(V \cdot n) \cdot n) n+\nabla_{\Gamma}(V \cdot n)\right] \\
& =\left(\nabla \phi_{\Gamma, \infty} \cdot n\right)(\nabla(V \cdot n) \cdot n)+\nabla_{\Gamma} \phi_{\Gamma, \infty} \cdot \nabla_{\Gamma}(V \cdot n) .
\end{aligned}
$$

Since $\llbracket \psi_{\mathrm{r}} \rrbracket_{\Gamma}=0$ and $\llbracket \varepsilon_{\Gamma} \nabla \phi_{\Gamma, \infty} \cdot n \rrbracket_{\Gamma}=0$, we thus have

$$
\begin{aligned}
& \llbracket \varepsilon_{\Gamma} \psi_{\mathrm{r}} \nabla \phi_{\Gamma, \infty} \cdot \nabla(V \cdot n) \rrbracket_{\Gamma} \\
& \quad=\llbracket \varepsilon_{\Gamma} \psi_{\mathrm{r}}\left(\nabla \phi_{\Gamma, \infty} \cdot n\right)(\nabla(V \cdot n) \cdot n) \rrbracket_{\Gamma}+\llbracket \varepsilon_{\Gamma} \psi_{\mathrm{r}} \nabla_{\Gamma} \phi_{\Gamma, \infty} \cdot \nabla_{\Gamma}(V \cdot n) \rrbracket_{\Gamma} \\
& \quad=\llbracket \varepsilon_{\Gamma} \alpha_{\Gamma} \cdot \nabla_{\Gamma}(V \cdot n) \rrbracket_{\Gamma} .
\end{aligned}
$$

One can verify that on both side of $\Gamma$

$$
\nabla_{\Gamma} \cdot\left((V \cdot n) \alpha_{\Gamma}\right)=(V \cdot n) \nabla_{\Gamma} \cdot \alpha_{\Gamma}+\alpha_{\Gamma} \cdot \nabla_{\Gamma}(V \cdot n) .
$$

Consequently, we have by (5.29), (5.39), (5.31), and the fact that $\nabla_{\Gamma} \phi_{\Gamma, \infty} \cdot n=0$ on each side of $\Gamma$ that

$$
\begin{aligned}
Q_{2,2} & =\int_{\Gamma} \llbracket \varepsilon_{\Gamma} \alpha_{\Gamma} \cdot \nabla_{\Gamma}(V \cdot n) \rrbracket_{\Gamma} d S \\
& =\int_{\Gamma} \llbracket \varepsilon_{\Gamma} \nabla_{\Gamma} \cdot\left((V \cdot n) \alpha_{\Gamma}\right) \rrbracket_{\Gamma} d S-\int_{\Gamma} \llbracket \varepsilon_{\Gamma}(V \cdot n) \nabla_{\Gamma} \cdot \alpha_{\Gamma} \rrbracket_{\Gamma} d S \\
& =\int_{\Gamma} \llbracket 2 \varepsilon_{\Gamma} H\left((V \cdot n) \alpha_{\Gamma} \cdot n\right) \rrbracket_{\Gamma} d S-\int_{\Gamma} \llbracket \varepsilon_{\Gamma} \nabla_{\Gamma} \cdot \alpha_{\Gamma} \rrbracket_{\Gamma}(V \cdot n) d S \\
& =-\int_{\Gamma} \llbracket \varepsilon_{\Gamma} \nabla_{\Gamma} \cdot \alpha_{\Gamma} \rrbracket_{\Gamma}(V \cdot n) d S .
\end{aligned}
$$

This, together with (5.29), 5.38), and the notation $\alpha_{\Gamma}=\psi_{\mathrm{r}} \nabla_{\Gamma} \phi_{\Gamma, \infty}$, implies that

$$
Q_{2}=-\int_{\Gamma} \llbracket \varepsilon_{\Gamma} \nabla \cdot \alpha_{\Gamma} \rrbracket_{\Gamma}(V \cdot n) d S=-\int_{\Gamma} \llbracket \varepsilon_{\Gamma} \nabla \cdot\left(\psi_{\mathrm{r}} \nabla_{\Gamma} \phi_{\Gamma, \infty}\right) \rrbracket_{\Gamma}(V \cdot n) d S .
$$


Now, let us calculate the term $Q_{3}$ in (5.26). Since $V=(V \cdot n) n$ (cf. (5.24)), we have from both sides of $\Gamma$ that

$$
\begin{aligned}
\nabla\left(\nabla \phi_{\Gamma, \infty} \cdot V\right) \cdot n & =\nabla\left(\left(\nabla \phi_{\Gamma, \infty} \cdot n\right)(V \cdot n)\right) \cdot n \\
& =\nabla\left(\nabla \phi_{\Gamma, \infty} \cdot n\right) \cdot n(V \cdot n)+\left(\nabla \phi_{\Gamma, \infty} \cdot n\right) \nabla(V \cdot n) \cdot n .
\end{aligned}
$$

Since $\llbracket \varepsilon_{\Gamma} \nabla \phi_{\Gamma, \infty} \cdot n \rrbracket_{\Gamma}=0$, we have by (5.26) and (5.35) that

$$
Q_{3}=\int_{\Gamma} \llbracket \varepsilon_{\Gamma} \psi_{\mathrm{r}} \nabla\left(\nabla \phi_{\Gamma, \infty} \cdot V\right) \cdot n \rrbracket_{\Gamma} d S=\int_{\Gamma} \llbracket \varepsilon_{\Gamma} \psi_{\mathrm{r}} \nabla\left(\nabla \phi_{\Gamma, \infty} \cdot n\right) \cdot n \rrbracket_{\Gamma}(V \cdot n) d S .
$$

It now follows from (5.26), (5.27), 5.40), and (5.41) that

$$
Q=\int_{\Gamma} \llbracket \varepsilon_{\Gamma}\left[\partial_{n} \phi_{\Gamma, \infty} \partial_{n} \psi_{\mathrm{r}}-\nabla \cdot\left(\psi_{\mathrm{r}} \nabla_{\Gamma} \phi_{\Gamma, \infty}\right)-\psi_{\mathrm{r}} \nabla\left(\nabla \phi_{\Gamma, \infty} \cdot n\right) \cdot n\right] \rrbracket_{\Gamma}(V \cdot n) d S .
$$

By the definition of the tangential gradient, the fact that $\Delta \phi_{\Gamma, \infty}=0$ on both sides of $\Gamma$ (cf. (2.8), and $\nabla \cdot n=2 H$ on $\Gamma$, we can simplify the terms inside the pair of brackets in (5.42). On both sides of $\Gamma$, we have

$$
\begin{aligned}
\partial_{n} \phi_{\Gamma, \infty} \partial_{n} \psi_{\mathrm{r}}-\nabla \cdot\left(\psi_{\mathrm{r}} \nabla_{\Gamma} \phi_{\Gamma, \infty}\right)-\psi_{\mathrm{r}} \nabla\left(\nabla \phi_{\Gamma, \infty} \cdot n\right) \cdot n \\
=\partial_{n} \phi_{\Gamma, \infty} \partial_{n} \psi_{\mathrm{r}}-\nabla \cdot\left[\psi_{\mathrm{r}} \nabla \phi_{\Gamma, \infty}-\psi_{\mathrm{r}}\left(\nabla \phi_{\Gamma, \infty} \cdot n\right) n\right]-\psi_{\mathrm{r}} \nabla\left(\nabla \phi_{\Gamma, \infty} \cdot n\right) \cdot n \\
=\partial_{n} \phi_{\Gamma, \infty} \partial_{n} \psi_{\mathrm{r}}-\nabla \psi_{\mathrm{r}} \cdot \nabla \phi_{\Gamma, \infty}-\psi_{\mathrm{r}} \Delta \phi_{\Gamma, \infty} \\
\quad \quad+\nabla\left(\psi_{\mathrm{r}}\left(\nabla \phi_{\Gamma, \infty} \cdot n\right)\right) \cdot n+\psi_{\mathrm{r}}\left(\nabla \phi_{\Gamma, \infty} \cdot n\right)(\nabla \cdot n)-\psi_{\mathrm{r}} \nabla\left(\nabla \phi_{\Gamma, \infty} \cdot n\right) \cdot n \\
=\partial_{n} \phi_{\Gamma, \infty} \partial_{n} \psi_{\mathrm{r}}-\nabla \psi_{\mathrm{r}} \cdot \nabla \phi_{\Gamma, \infty}+\left(\nabla \phi_{\Gamma, \infty} \cdot n\right)\left(\nabla \psi_{\mathrm{r}} \cdot n\right)+\psi_{\mathrm{r}}\left(\nabla \phi_{\Gamma, \infty} \cdot n\right)(\nabla \cdot n) \\
=2 \partial_{n} \phi_{\Gamma, \infty} \partial_{n} \psi_{\mathrm{r}}-\left[\left(\nabla \psi_{\mathrm{r}} \cdot n\right) n+\nabla_{\Gamma} \psi_{\mathrm{r}}\right]\left[\left(\nabla \phi_{\Gamma, \infty} \cdot n\right) n+\nabla_{\Gamma} \phi_{\Gamma, \infty}\right]+2 H \psi_{\mathrm{r}} \partial_{n} \phi_{\Gamma, \infty} \\
=\partial_{n} \phi_{\Gamma, \infty} \partial_{n} \psi_{\mathrm{r}}-\nabla_{\Gamma} \phi_{\Gamma, \infty} \cdot \nabla_{\Gamma} \psi_{\mathrm{r}}+2 H \psi_{\mathrm{r}} \partial_{n} \phi_{\Gamma, \infty} .
\end{aligned}
$$

Plug this into 5.42. Noting that $\psi_{\mathrm{r}}=\psi_{\Gamma}-\hat{\phi}_{\Gamma, \infty}$ and that all $\nabla_{\Gamma} \psi_{\mathrm{r}}, \nabla_{\Gamma} \phi_{\Gamma, \infty}, \varepsilon_{\Gamma} \partial_{n}\left(\psi_{\Gamma}-\hat{\phi}_{\Gamma, \infty}\right)$, and $\varepsilon_{\Gamma} \partial_{n} \phi_{\Gamma, \infty}$ are continuous across the boundary $\Gamma$, we obtain that

$$
\begin{aligned}
Q & =\int_{\Gamma} \llbracket \varepsilon_{\Gamma}\left(\partial_{n} \psi_{\mathrm{r}} \partial_{n} \phi_{\Gamma, \infty}-\nabla_{\Gamma} \psi_{\mathrm{r}} \cdot \nabla_{\Gamma} \phi_{\Gamma, \infty}\right) \rrbracket_{\Gamma}(V \cdot n) d S \\
& =\int_{\Gamma} \llbracket \varepsilon_{\Gamma}\left[\partial_{n}\left(\psi_{\Gamma}-\hat{\phi}_{\Gamma, \infty}\right) \partial_{n} \phi_{\Gamma, \infty}-\nabla_{\Gamma}\left(\psi_{\Gamma}-\hat{\phi}_{\Gamma, \infty}\right) \cdot \nabla_{\Gamma} \phi_{\Gamma, \infty}\right] \rrbracket_{\Gamma}(V \cdot n) d S .
\end{aligned}
$$

Finally, we obtain by (5.23), (5.26), and (5.43) that some of the terms in $\delta_{\Gamma, V} E[\Gamma](5.23)$ are simplified into

$$
\begin{aligned}
& \frac{1}{2} \int_{\Gamma} \llbracket \varepsilon_{\Gamma} \partial_{n} \hat{\phi}_{\Gamma, \infty} \partial_{n} \phi_{\Gamma, \infty} \rrbracket_{\Gamma}(V \cdot n) d S-\frac{1}{2} \int_{\Gamma} \llbracket \varepsilon_{\Gamma} \nabla_{\Gamma} \hat{\phi}_{\Gamma, \infty} \cdot \nabla_{\Gamma} \phi_{\Gamma, \infty} \rrbracket_{\Gamma}(V \cdot n) d S \\
& \quad+\int_{\Omega_{+}} \frac{1}{2}\left(\zeta_{\Gamma, V}-\nabla \phi_{\Gamma, \infty} \cdot V\right) B^{\prime}\left(\psi_{\Gamma}-\frac{\phi_{\Gamma, \infty}}{2}\right) d X
\end{aligned}
$$




$$
\begin{aligned}
= & \frac{1}{2} \int_{\Gamma} \llbracket \varepsilon_{\Gamma} \partial_{n} \hat{\phi}_{\Gamma, \infty} \partial_{n} \phi_{\Gamma, \infty} \rrbracket_{\Gamma}(V \cdot n) d S-\frac{1}{2} \int_{\Gamma} \llbracket \varepsilon_{\Gamma} \nabla_{\Gamma} \hat{\phi}_{\Gamma, \infty} \cdot \nabla_{\Gamma} \phi_{\Gamma, \infty} \rrbracket_{\Gamma}(V \cdot n) d S+\frac{1}{2} Q \\
= & \frac{1}{2} \int_{\Gamma} \llbracket \varepsilon_{\Gamma}\left(\partial_{n} \psi_{\Gamma} \partial_{n} \phi_{\Gamma, \infty}-\nabla_{\Gamma} \psi_{\Gamma} \cdot \nabla_{\Gamma} \phi_{\Gamma, \infty}\right) \rrbracket_{\Gamma}(V \cdot n) d S \\
= & \frac{1}{2} \int_{\Gamma} \varepsilon_{+} \partial_{n} \psi_{\Gamma}^{+} \partial_{n} \phi_{\Gamma, \infty}^{+}(V \cdot n) d S-\frac{1}{2} \int_{\Gamma} \varepsilon_{-} \partial_{n} \psi_{\Gamma}^{-} \partial_{n} \phi_{\Gamma, \infty}^{-}(V \cdot n) d S \\
& -\frac{\varepsilon_{+}}{2} \int_{\Gamma} \nabla_{\Gamma} \psi_{\Gamma} \cdot \nabla_{\Gamma} \phi_{\Gamma, \infty}(V \cdot n) d S+\frac{\varepsilon_{-}}{2} \int_{\Gamma} \nabla_{\Gamma} \psi_{\Gamma} \cdot \nabla_{\Gamma} \phi_{\Gamma, \infty}(V \cdot n) d S \\
= & \frac{1}{2}\left(\frac{1}{\varepsilon_{+}}-\frac{1}{\varepsilon_{-}}\right) \int_{\Gamma} \varepsilon_{\Gamma} \partial_{n} \psi_{\Gamma} \varepsilon_{\Gamma} \partial_{n} \phi_{\Gamma, \infty}(V \cdot n) d S-\frac{\varepsilon_{+}-\varepsilon_{-}}{2} \int_{\Gamma} \nabla_{\Gamma} \psi_{\Gamma} \cdot \nabla_{\Gamma} \phi_{\Gamma, \infty}(V \cdot n) d S .
\end{aligned}
$$

This and (5.23) imply the desired formula (3.8). The proof is complete.

Acknowledgments. BL was supported in part by the US National Science Foundation through the grant DMS-1913144, the US National Institutes of Health through the grant R01GM132106, and a 2019-2020 Lattimer Research Fellowship, Division of Physical Sciences, University of California, San Diego. ZZ was supported in part by the Natural Science Foundation of Zhejiang Province, China, through the grant LY17A010029. SZ was supported in part by the National Natural Science Foundation of China (NSFC) through the grant NSFC 21773165, the Natural Science Foundation of Jiangsu Province, China, through the grant BK20160302, and the Young Elite Scientist Sponsorship Program, Jiangsu Association for Science and Technology, China.

\section{References}

[1] R. Adams. Sobolev Spaces. Academic Press, New York, 1975.

[2] D. Andelman. Electrostatic properties of membranes: The Poisson-Boltzmann theory. In R. Lipowsky and E. Sackmann, editors, Handbook of Biological Physics, volume 1, pages 603-642. Elsevier, 1995.

[3] D. Bucur and G. Buttazzo. Variational Methods in Shape Optimization Problems. Progress in Nonlinear Differential Equations and Their Applications. Birkhäuser, Boston, 2005.

[4] Q. Cai, X. Ye, and R. Luo. Dielectric pressure in continuum electrostatic solvation of biomolecules. Phys. Chem. Chem. Phys., 14:15917-15925, 2012.

[5] Q. Cai, X. Ye, J. Wang, and R. Luo. Dielectric boundary forces in numerical PoissonBoltzmann methods: Theory and numerical strategies. Chem. Phys. Lett., 514:368-373, 2011. 
[6] D. L. Chapman. A contribution to the theory of electrocapillarity. Phil. Mag., 25:475481, 1913.

[7] J. Che, J. Dzubiella, B. Li, and J. A. McCammon. Electrostatic free energy and its variations in implicit solvent models. J. Phys. Chem. B, 112:3058-3069, 2008.

[8] L. Cheng, B. Li, M. White, and S. Zhou. Motion of a cylindrical dielectric boundary. SIAM J. Applied Math., 73:594-616, 2013.

[9] L.-T. Cheng, J. Dzubiella, J. A. McCammon, and B. Li. Application of the level-set method to the implicit solvation of nonpolar molecules. J. Chem. Phys., 127:084503, 2007.

[10] L.-T. Cheng, Z. Wang, P. Setny, J. Dzubiella, B. Li, and J. A. McCammon. Interfaces and hydrophobic interactions in receptor-ligand systems: A level-set variational implicit solvent approach. J. Chem. Phys., 131:144102, 2009.

[11] L.-T. Cheng, Y. Xie, J. Dzubiella, J. A. McCammon, J. Che, and B. Li. Coupling the level-set method with molecular mechanics for variational implicit solvation of nonpolar molecules. J. Chem. Theory Comput., 5:257-266, 2009.

[12] M. Chipot, D. Kinderlehrer, and G. V. Caffarelli. Smoothness of linear laminates. Arch. Rational Mech. Anal., 96:81-96, 1986.

[13] B. Chu. Molecular Forces, Based on the Lecture of Peter J. W. Debye. Interscience, John Wiley \& Sons, 1967.

[14] C. J. Cramer and D. G. Truhlar. Implicit solvation models: Equilibria, structure, spectra, and dynamics. Chem. Rev., 99:2161-2200, 1999.

[15] S. Dai, B. Li, and J. Lu. Convergence of phase-field free energy and boundary force for molecular solvation. Arch. Rational Mech. Anal., 227(1):105-147, 2018.

[16] M. E. Davis and J. A. McCammon. Electrostatics in biomolecular structure and dynamics. Chem. Rev., 90:509-521, 1990.

[17] P. Debye and E. Hückel. Zur theorie der elektrolyte. Physik. Zeitschr., 24:185-206, 1923.

[18] M. C. Delfour and J.-P. Zolésio. Shapes and Geometries: Analysis, Differential Calculus, and Optimization. SIAM, 1987.

[19] J. Dzubiella, J. M. J. Swanson, and J. A. McCammon. Coupling hydrophobicity, dispersion, and electrostatics in continuum solvent models. Phys. Rev. Lett., 96:087802, 2006.

[20] J. Dzubiella, J. M. J. Swanson, and J. A. McCammon. Coupling nonpolar and polar solvation free energies in implicit solvent models. J. Chem. Phys., 124:084905, 2006. 
[21] J. Elschner, J. Rehberg, and G. Schmidt. Optimal regularity for elliptic transmission problems including $C^{1}$ interfaces. Interfaces and Free Boundaries, 9:233-252, 2007.

[22] L. C. Evans. Partial Differential Equations, volume 19 of Graduate Studies in Mathematics. Amer. Math. Soc., 2nd edition, 2010.

[23] F. Fixman. The Poisson-Boltzmann equation and its application to polyelecrolytes. $J$. Chem. Phys., 70:4995-5005, 1979.

[24] F. Fogolari and J. M. Briggs. On the variational approach to Poisson-Boltzmann free energies. Chem. Phys. Lett., 281:135-139, 1997.

[25] D. Gilbarg and N. S. Trudinger. Elliptic Partial Differential Equations of Second Order. Springer-Verlag, 2nd edition, 1998.

[26] J. Ginster and P. Gladbach. Many-particle limits in molecular solvation. Arch. Rational Mech. Anal., 235:793-839, 2020.

[27] M. Gouy. Sur la constitution de la charge électrique a la surface d'un électrolyte. J. de Phys., 9:457-468, 1910.

[28] J. Huang and J. Zou. Some new a priori estimates for second-order elliptic and parabolic interface problems. J. Diff. Equations, 184:570-586, 2002.

[29] J. Huang and J. Zou. Uniform a priori estimates for elliptic and static Maxwell interface problems. Disc. Cont. Dyn. Syst. B, 7(1):145-170, 2007.

[30] B. Kawohl, O. Pironneau, L. Tartar, and J.-P. Zolésio. Optimal Shape Design, volume 1740 of Lecture Notes in Mathematics. Springer, 2000.

[31] S. G. Krantz and H. R. Parks. Distance to $C^{k}$ hypersurfaces. J. Diff. Equations, 40:116$120,1981$.

[32] O. A. Ladyzhenskaya and N. N. Ural'tseva. Linear and Quasilinear Elliptic Equations, volume 46 of Mathematics in Science and Engeneering. Academic Press, 1968.

[33] B. Li. Variational properties of unbounded order parameters. SIAM J. Math. Anal., 38(1):16-36, 2006.

[34] B. Li. Minimization of electrostatic free energy and the Poisson-Boltzmann equation for molecular solvation with implicit solvent. SIAM J. Math. Anal., 40:2536-2566, 2009. See also an erratum in SIAM. J. Math. Anal., 43, 2776-2777, 2011.

[35] B. Li, X. Cheng, and Z. Zhang. Dielectric boundary force in molecular solvation with the Poisson-Boltzmann free energy: A shape derivative approach. SIAM J. Applied Math., 71:2093-2111, 2011. 
[36] B. Li, P. Liu, Z. Xu, and S. Zhou. Ionic size effects: Generalized Boltzmann distributions, counterion stratification, and modified Debye length. Nonlinearity, 26:2899-2922, 2013.

[37] B. Li and Y. Liu. Diffused solute-solvent interface with Poisson-Boltzmann electrostatics: Free-energy variation and sharp-interface limit. SIAM J. Applied Math, 75(5):2072-2092, 2015.

[38] B. Li., H. Sun, and S. Zhou. Stability of a cylindrical solute-solvent interface: Effect of geometry, electrostatics, and hydrodynamics. SIAM J. Applied Math., 75:907-928, 2015.

[39] Y. Y. Li and M. Vogelius. Gradient estimates for solutions to divergence form elliptic equations with discontinuous coefficients. Arch. Rational Mech. Anal., 153:91-151, 2000.

[40] W. Littman, G. Stampacchia, and H. F. Weinberger. Regular points for elliptic equations with discontinuous coefficients. Ann. Scuola Norm. Sup. Pisa, 3:43-77, 1963.

[41] X. Liu, Y. Qiao, and B. Z. Lu. Analysis of the mean field free energy functional of electrolyte solution with nonhomogenous boundary conditions and the generalized PB/PNP equations with inhomogeneous dielectric permittivity. SIAM J. Appl. Math., 78(2):11311154, 2018.

[42] J. Lu and F. Otto. Nonexistence of a minimizer for Thomas-Fermi-Dirac-von Weizsäcker model. Commun. Pure Appl. Math., 67(10):1605-1617, 2014.

[43] M. Mikucki and Y. C. Zhou. Electrostatic forces on charged surfaces of bilayer lipid membranes. SIAM J. Applied Math, 74:1-21, 2014.

[44] E. S. Reiner and C. J. Radke. Variational approach to the electrostatic free energy in charged colloidal suspensions: general theory for open systems. J. Chem. Soc. Faraday Trans., 86:3901-3912, 1990.

[45] K. A. Sharp and B. Honig. Electrostatic interactions in macromolecules: Theory and applications. Annu. Rev. Biophys. Biophys. Chem., 19:301-332, 1990.

[46] J. Sokolowski and J.-P. Zolésio. Introduction to Shape Optimization: Shape Sensitivity Analysis. Springer Series in Computational Mathematics. Springer, 1992.

[47] H. Sun, J. Wen, Y. Zhao, B. Li, and J. A. McCammon. A self-consistent phase-field approach to implicit solvation of charged molecules with Poisson-Boltzmann electrostatics. J. Chem. Phys., 143:243110, 2015.

[48] J. Tomasi and M. Persico. Molecular interactions in solution: An overview of methods based on continuous distributions of the solvent. Chem. Rev., 94:2027-2094, 1994.

[49] Z. Wang, J. Che, L.-T. Cheng, J. Dzubiella, B. Li, and J. A. McCammon. Level-set variational implicit solvation with the Coulomb-field approximation. J. Chem. Theory Comput., 8:386-397, 2012. 
[50] L. Xiao, Q. Cai, X. Ye, J. Wang, and R. Luo. Electrostatic forces in the PoissonBoltzmann systems. J. Chem. Phys., 139:094106, 2013.

[51] H. Yin, G. Feng, G. M. Clore, G. Hummer, and J. C. Rasaiah. Water in the polar and nonpolar cavities of the protein interleukin-1ß. J. Phys. Chem. B, 114:16290-16297, 2010.

[52] H. Yin, G. Hummer, and J. C. Rasaiah. Metastable water clusters in the nonpolar cavities of the thermostable protein tetrabrachion. J. Amer. Chem. Soc., 129:7369-7377, 2007.

[53] S. Zhou, L.-T. Cheng, J. Dzubiella, B. Li, and J. A. McCammon. Variational implicit solvation with Poisson-Boltzmann theory. J. Chem. Theory Comput., 10:1454-1467, 2014.

[54] S. Zhou, R. G. Weiß, L.-T. Cheng, J. Dzubiella, J. A. McCammon, and B. Li. Variational implicit-solvent predictions of the dry-wet transition pathways for ligand-receptor binding and unbinding kinetics. Proc. Natl Acad. Sci. USA, 116(30):14989-14994, 2019. 